Supporting Information

Mechanism of the Iron(II)-Catalyzed Hydrosilylation of Ketones: Activation of Iron Carboxylate Precatalysts and Reaction Pathways of the Active Catalyst Tim Bleith and Lutz H. Gade*

\title{
Contents:
}

\section{General Information}

II. Preparation of the Complexes and Reagents

III. Details for Kinetic Experiments

IV. Details for Mechanistic Experiments

V. Computational Details

VI. NMR Spectra of New Compounds $\left({ }^{1} \mathrm{H}\right.$ NMR, ${ }^{13} \mathrm{C}$ NMR, ${ }^{19}$ F NMR) 


\section{General Information}

All manipulations were carried out using standard Schlenk line or drybox techniques under an atmosphere of argon. Solvents were dried over activated alumina columns using a solvent purification system (M. Braun SPS 800) or according to standard literature-known methods ${ }^{1}$ and stored in glass ampules under an argon atmosphere. ${ }^{1} \mathrm{H}$, ${ }^{13} \mathrm{C}\left\{{ }^{1} \mathrm{H}\right\},{ }^{19} \mathrm{~F},{ }^{29} \mathrm{Si}$ NMR spectra were recorded on a Bruker Avance III 600, a Bruker

Avance II 400 or a Bruker DRX 200 spectrometer. ${ }^{1} \mathrm{H}$ and ${ }^{13} \mathrm{C}$ NMR spectra were referenced internally to residual protio-solvent $\left({ }^{1} \mathrm{H}\right)$ or solvent $\left({ }^{13} \mathrm{C}\right)$ resonances and are reported relative to tetramethylsilane. ${ }^{19} \mathrm{~F}$ and ${ }^{29} \mathrm{Si}$ NMR spectra were referenced to an external standard (the shifts of $\mathrm{CFCl}_{3}$ or tetramethylsilane, respectively, were set to $0 \mathrm{ppm})$. HPLC analyses were conducted on an Agilent 1200 Series chromatograph using chiral Daicel columns (AD-H, OD-H, OJ-H) according to the methods previously reported ${ }^{2}$ or on an achiral Macherey-Nagel column (Nucleodur 100-5). Mass spectra were recorded by the mass spectrometry service of the University of Heidelberg Organic Chemistry Laboratory and the elemental analyses were measured by the analytical services of the University of Heidelberg. boxmiH ligand, ${ }^{3} \mathrm{FeCl}_{2}(\mathrm{py})_{4}{ }^{4}$ $\mathrm{Fe}$ (boxmi)OAc (3), ${ }^{5} \mathrm{Fe}$ (boxmi)Cl, ${ }^{5} \mathrm{Fe}$ (boxmi) $\mathrm{CH}_{2} \mathrm{TMS}(4),{ }^{2} \mathrm{Fe}$ (boxmi) $\mathrm{OCHCH}_{3} \mathrm{Ph}$ $(5),{ }^{2}$ radical clock $6,{ }^{6}$ and its corresponding alcohol $7^{7}$ were synthesized according to the literature procedures. All other reagents were commercially available and used as received. Liquids were degassed by three freeze-pump-thaw cycles before use. All iron salts were purchased with a trace metal purity of $99.99 \%$ or higher. 


\section{Preparation of the Complexes and Reagents}

Procedure for the synthesis of $\mathrm{Fe}(\mathrm{boxmi}) \mathrm{OAc}-{ }^{13} \mathrm{C}_{1}\left(3-{ }^{13} \mathrm{C}\right)$ :
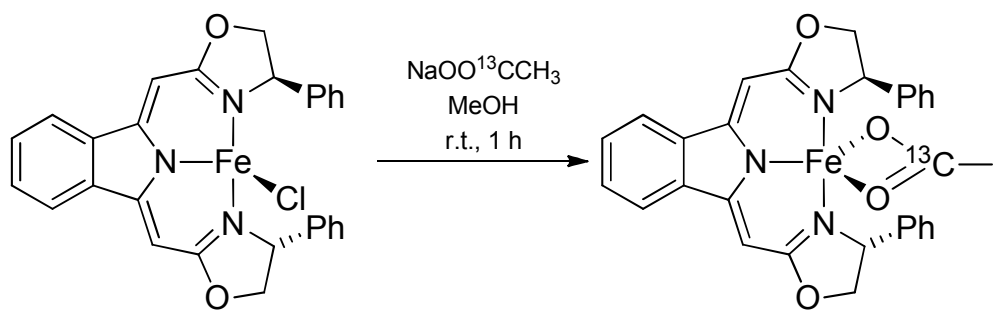

$\mathrm{Fe}($ boxmi)Cl (152 mg, $0.29 \mathrm{mmol})$ and $\mathrm{NaOAc}-{ }^{13} \mathrm{C}_{1}(33.7 \mathrm{mg}, 0.41 \mathrm{mmol})$ were dissolved in $7 \mathrm{~mL}$ abs. $\mathrm{MeOH}$ and stirred for $20 \mathrm{~min}$ at room temperature. The dark solution was evaporated to dryness and the residue was extracted with toluene three times and filtered over Celite. After evaporation and drying in vacuo, $87.2 \mathrm{mg}$ (52\% yield) of a black powder were obtained. Further purification was achieved by recrystallization from toluene/pentane mixtures.

$\operatorname{HRMS}\left(\mathrm{FAB}^{+}\right)$: Anal. for $\mathrm{C}_{29}{ }^{13} \mathrm{CH}_{25} \mathrm{~N}_{3} \mathrm{O}_{4} \mathrm{Fe}\left(\mathrm{M}^{+}\right)$Calcd.: 548.1228, Found: 548.1242. Anal. calcd. for $\mathrm{C}_{29}{ }^{13} \mathrm{CH}_{25} \mathrm{~N}_{3} \mathrm{O}_{4} \mathrm{Fe}$ : C, 65.89; H, 4.60; N, 7.66. Found: C, 65.45; H, $5.08 ; \mathrm{N}, 7.12$.

Procedure for the synthesis of $\mathrm{Fe}(\mathrm{boxmi}) \mathrm{OAc}-\mathrm{D}_{3}\left(3-\mathrm{D}_{3}\right)$ :

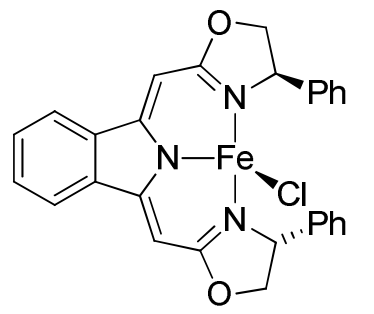

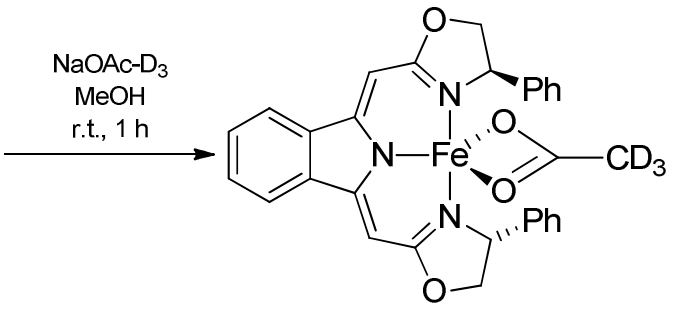

$\mathrm{Fe}($ boxmi)Cl (186 mg, $0.36 \mathrm{mmol})$ and $\mathrm{NaOAc}-\mathrm{D}_{3}(62.0 \mathrm{mg}, 0.73 \mathrm{mmol})$ were dissolved in $24 \mathrm{~mL}$ abs. $\mathrm{MeOH}$ and stirred for $2.5 \mathrm{~h}$ at room temperature. The dark solution was evaporated to dryness and the residue was extracted with toluene three times and filtered over Celite. After evaporation and drying in vacuo, $154.2 \mathrm{mg}$ (79\% yield) 
of a black powder were obtained. Further purification was achieved by recrystallization from toluene/pentane mixtures.

${ }^{2} \mathrm{H}$ NMR (92.12 MHz, toluene, $\left.295 \mathrm{~K}\right): \delta$ (ppm) 15.5 (FWHM $45 \mathrm{~Hz}$ ).

HRMS(FAB $\left.{ }^{+}\right)$: Anal. for $\mathrm{C}_{30} \mathrm{H}_{22} \mathrm{D}_{3} \mathrm{~N}_{3} \mathrm{O}_{4} \mathrm{Fe}\left(\mathrm{M}^{+}\right)$Calcd.: 550.1383, Found: 550.1348.

\section{Procedure for the synthesis of (EtO $)_{2} \mathrm{MeSiD}$ :}

$$
(\mathrm{EtO})_{2} \mathrm{MeSiH}+\mathrm{D}_{2}(15 \text { bar }) \underset{\text { tetraglyme, r.t. }}{\stackrel{\left[\mathrm{Rh}\left(\mathrm{PPh}_{3}\right)_{3} \mathrm{Cl}\right.}{\longrightarrow}}(\mathrm{EtO})_{2} \mathrm{MeSiD}
$$

(EtO) $)_{2} \mathrm{MeSiH}\left(2.5 \mathrm{~mL}, 15.6 \mathrm{mmol}\right.$, degassed) and $\mathrm{Rh}\left(\mathrm{PPh}_{3}\right)_{3} \mathrm{Cl}(1.01 \mathrm{~g}, 1.1 \mathrm{mmol})$ were suspended in tetraglyme $(15 \mathrm{~mL}$, dried over $\mathrm{Na}$, degassed). Under vigorous stirring, the mixture was pressurized with 15 bar $\mathrm{D}_{2}$ for 7 days, while replacing the atmosphere every 2 days. The product was separated using a bulb to bulb distillation. $1.1 \mathrm{~g}$ (8.1 mmol, 52\% yield) of a colorless liquid were obtained. The degree of deuteration was determined using ${ }^{1} \mathrm{H}$ NMR spectroscopy and was found to higher than $98 \%$.

${ }^{1} \mathrm{H}$ NMR (600.13 MHz, $\left.\mathrm{CDCl}_{3}, 295 \mathrm{~K}\right): \delta(\mathrm{ppm})$ 3.82-3.77 (m, 4H, OCH$), 1.22(\mathrm{t}$, $\left.{ }^{3} J_{\mathrm{HH}}=7.0 \mathrm{~Hz}, 6 \mathrm{H}, \mathrm{OCH}_{2} \mathrm{CH}_{3}\right), 0.19\left(\mathrm{~s}, 3 \mathrm{H}, \mathrm{SiCH}_{3}\right)$.

${ }^{2} \mathrm{H}$ NMR (92.12 MHz, $\left.\mathrm{CHCl}_{3}, 295 \mathrm{~K}\right): \delta(\mathrm{ppm}) 4.62$.

${ }^{13} \mathrm{C} \mathrm{NMR}\left(150.92 \mathrm{MHz}, \mathrm{CDCl}_{3}, 295 \mathrm{~K}\right): \delta(\mathrm{ppm}) 59.24\left(\mathrm{OCH}_{2}\right), 18.37\left(\mathrm{OCH}_{2} \mathrm{CH}_{3}\right),-$ $3.15\left(\mathrm{t},{ }^{2} J_{\mathrm{DC}}=2.6 \mathrm{~Hz}, \mathrm{SiCH}_{3}\right)$.

${ }^{29} \mathrm{Si}$ NMR (79.44 MHz, $\left.\mathrm{CDCl}_{3}, 295 \mathrm{~K}\right): \delta(\mathrm{ppm})-16.18\left(\mathrm{t},{ }^{1} J_{\mathrm{Dsi}}=36.0 \mathrm{~Hz}\right)$.

HRMS (EI $\left.{ }^{+}\right): 135.0850\left(1.7 \%\right.$, calcd. for $\left.\left[\mathrm{M}^{+}\right]^{+}: 135.0826\right), 133.0676(100 \%$, calcd. for $[\mathrm{M}-\mathrm{D}]^{+}:$133.0685), $120.0576\left(83.3 \%\right.$, calcd. for $\left.\left[\mathrm{M}-\mathrm{CH}_{3}\right]^{+}: 120.0591\right), 90.0469$ (33.4\%, calcd. for $[\mathrm{M}-\mathrm{OEt}]^{+}:$90.0485). 


\section{Details for Kinetic Experiments}

\section{High Temperature Kinetics Using Fe(boxmi)OAc (3) as Catalyst}

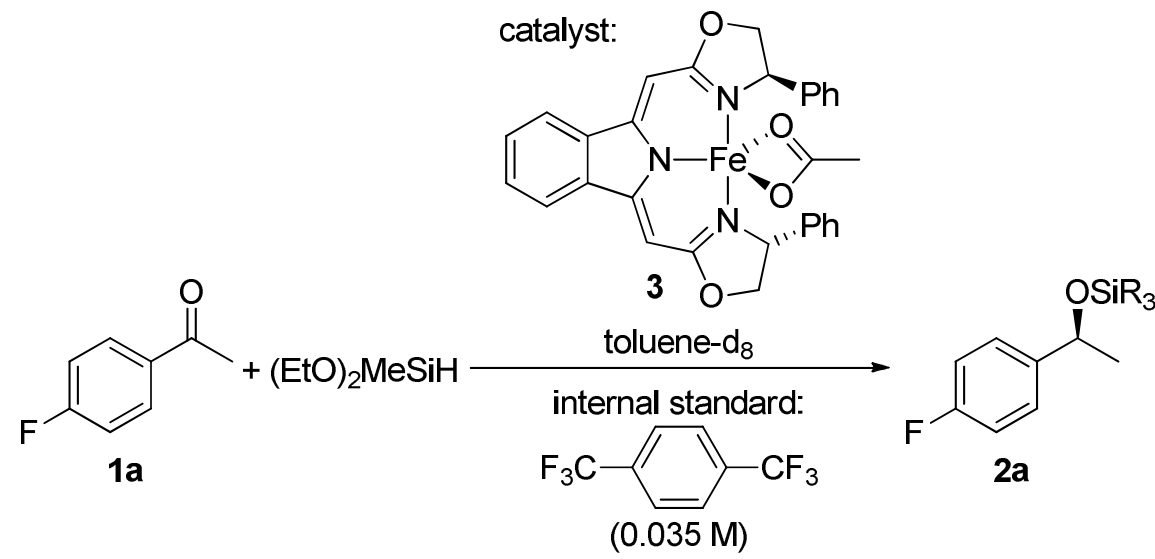

reaction conditions:

$\begin{array}{lll}\text { [Catalyst] } & 6.8 \times 10^{-4}-1.8 \times 10^{-2} \mathrm{M} & 6.0 \times 10^{-3} \mathrm{M} \\ \text { [Ketone] } & 0.060-0.75 \mathrm{M} & 0.15 \mathrm{M} \\ \left.\text { [(EtO) }{ }_{2} \mathrm{MeSiH}\right] & 0.075-0.45 \mathrm{M} & 0.30 \mathrm{M} \\ \text { Temperature } & 45-71^{\circ} \mathrm{C} & 65^{\circ} \mathrm{C}\end{array}$

$\mathrm{Fe}(\text { boxmi)OAc (3), 4'-fluoroacetophenone (1a), (EtO) })_{2} \mathrm{MeSiH}, \quad$ and 1,4bis(trifluoromethyl)benzene as internal standard were dissolved in $0.5 \mathrm{~mL}$ toluene- $\mathrm{d}_{8}$ in a J. Young NMR tube. The sample was heated to $65{ }^{\circ} \mathrm{C}$ within the NMR spectrometer and ${ }^{19} \mathrm{~F}$ NMR spectra were recorded until at least $70 \%$ conversion were observed. For analysis of the spectra, the integral of the internal standard was set to 1 in each spectrum and the values for the starting material as well as the products were used without further manipulation. 


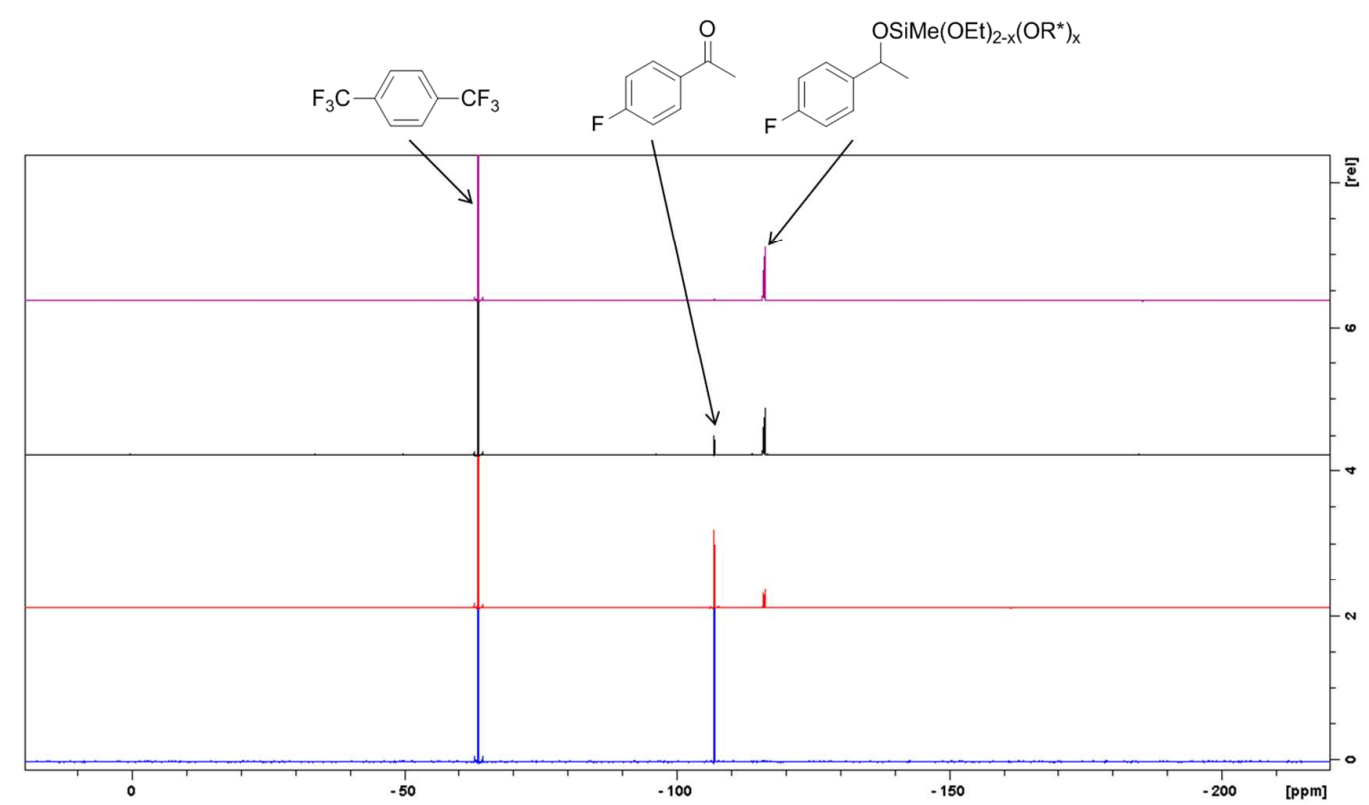

Figure S 1: Typical ${ }^{19}$ F NMR spectra recorded during a kinetic measurement run.

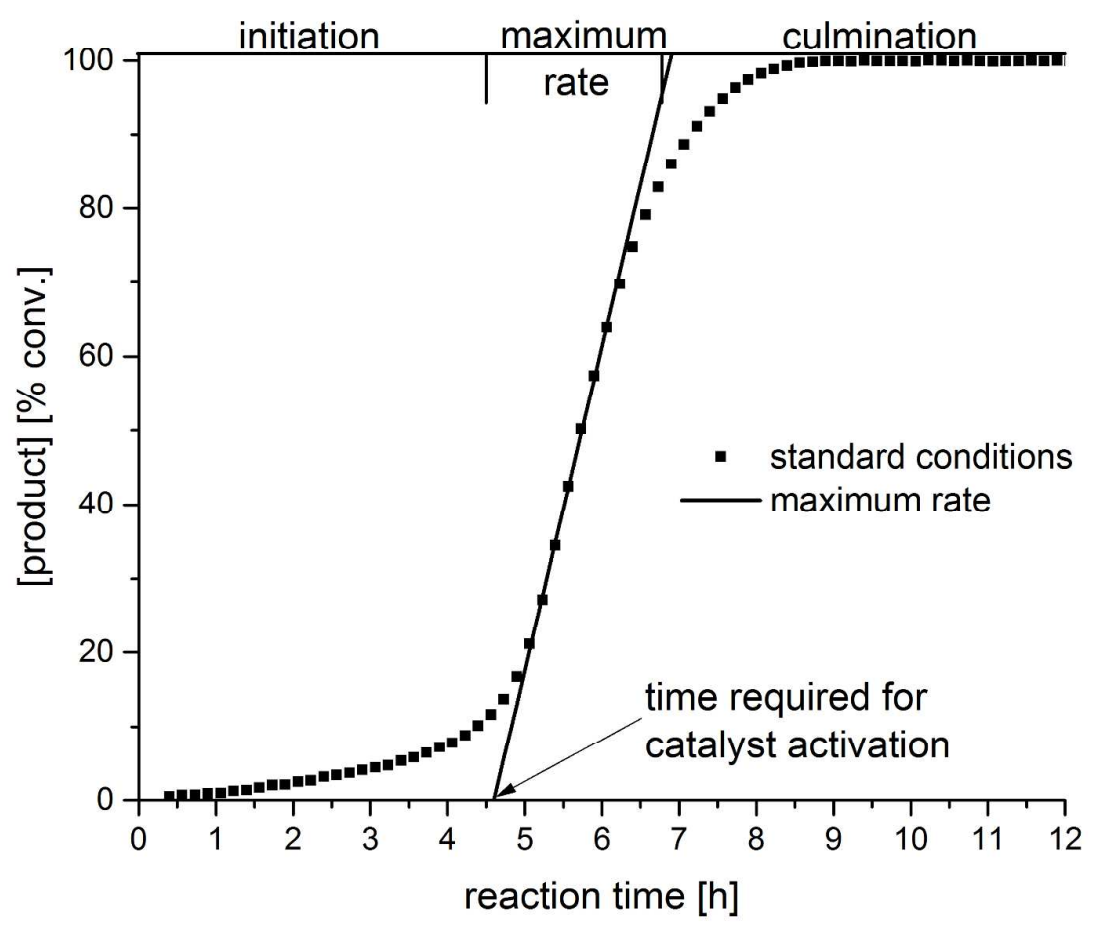

Figure S 2: Typical reaction profile for the catalytic hydrosilylation using $\mathbf{3}$ as catalyst. 

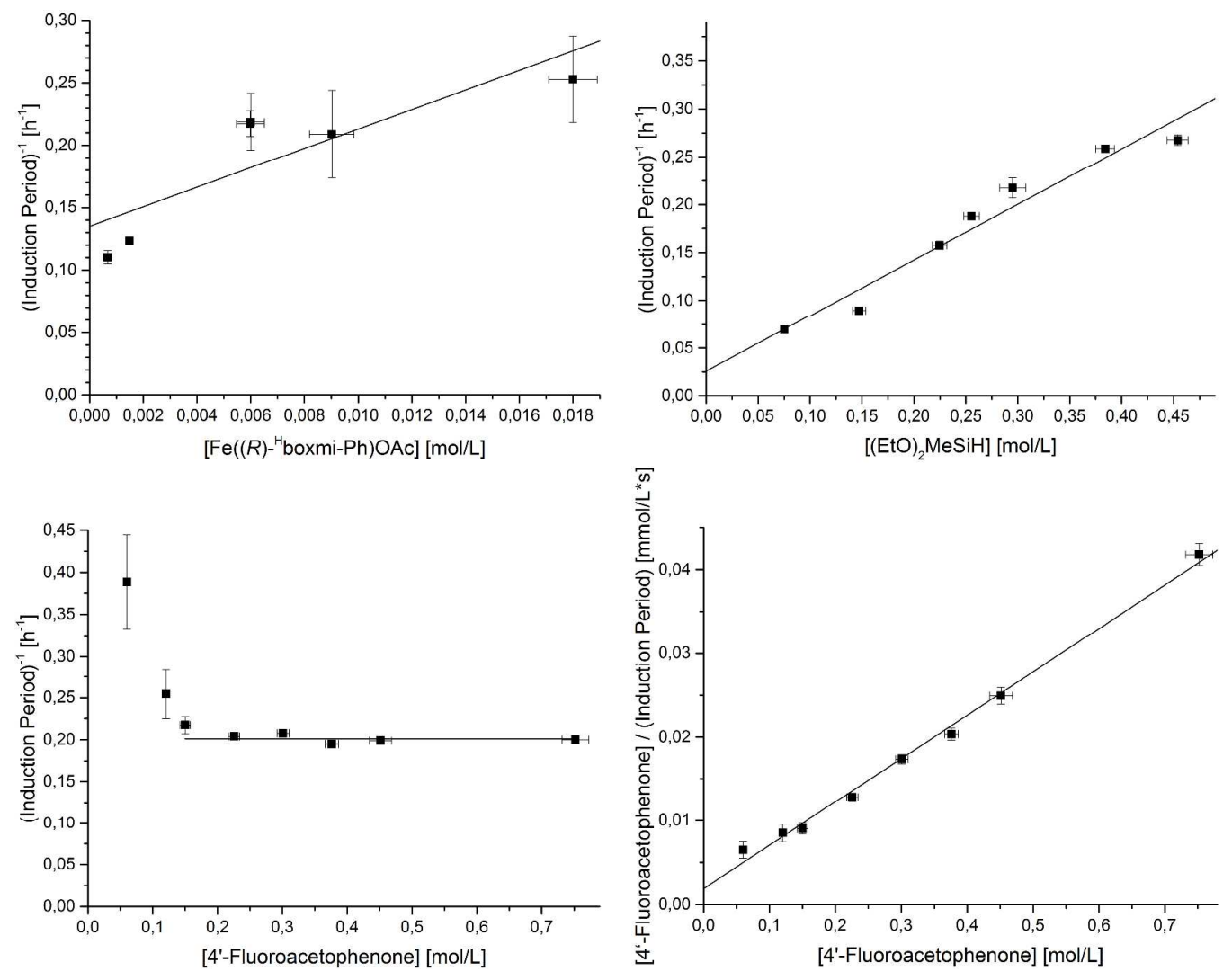

Figure S 3: Time of the induction period as a function of [catalyst], [(EtO $\left.)_{2} \mathrm{MeSiH}\right]$, and [ketone], respectively, for the catalytic hydrosilylation using $\mathrm{Fe}($ boxmi)OAc (3) as catalyst. 

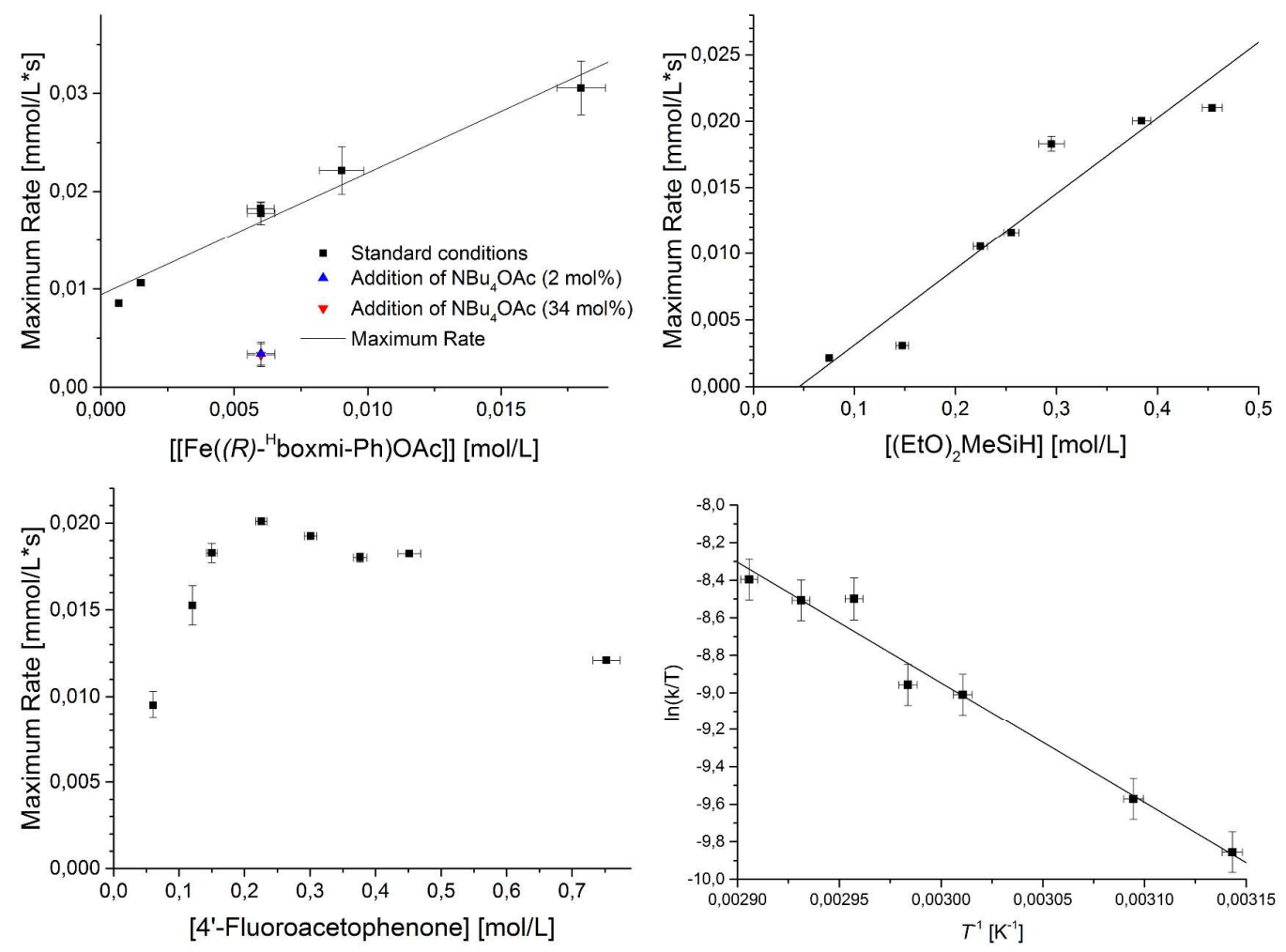

Figure S 4: Determining the rate law for the catalytic hydrosilylation using $\mathrm{Fe}($ boxmi)OAc (3) as catalyst. The rate law was found to be first order in [catalyst], $\left[(\mathrm{EtO}){ }_{2} \mathrm{MeSiH}\right]$, and [ketone], whereas the latter shows a saturation behavior for concentrations of $0.2 \mathrm{M}$ and above. From an Eyring plot, shown on the bottom right, the following parameters were derived: $\Delta H^{\dagger}=52 \pm 4 \mathrm{~kJ} \mathrm{~mol}^{-1}, \Delta S^{\dagger}=-112 \pm 11 \mathrm{~J} /(\mathrm{K} \cdot \mathrm{mol})$.

For all runs with induction ahead, iron complex 3, (EtO) $)_{2} \mathrm{MeSiH}$ and 1,4bis(trifluoro)benzene were dissolved in $0.5 \mathrm{~mL}$ toluene- $\mathrm{d}_{8}$ and heated in a J. Young NMR tube for 20 to $30 \mathrm{~h}$ to $65^{\circ} \mathrm{C}$ and then cooled to room temperature. Using this pretreatment, the highest reaction rates were obtained reproducibly (see Figure S 5). In an argon filled glovebox, the NMR tube was opened to add 4'-fluoroacetophenone (1a) and the reaction tube was then transferred to a preheated NMR spectrometer $\left(65^{\circ} \mathrm{C}\right)$ and ${ }^{19} \mathrm{~F}$ NMR spectra were recorded. Data analysis was performed consistently with the procedure previously described. 


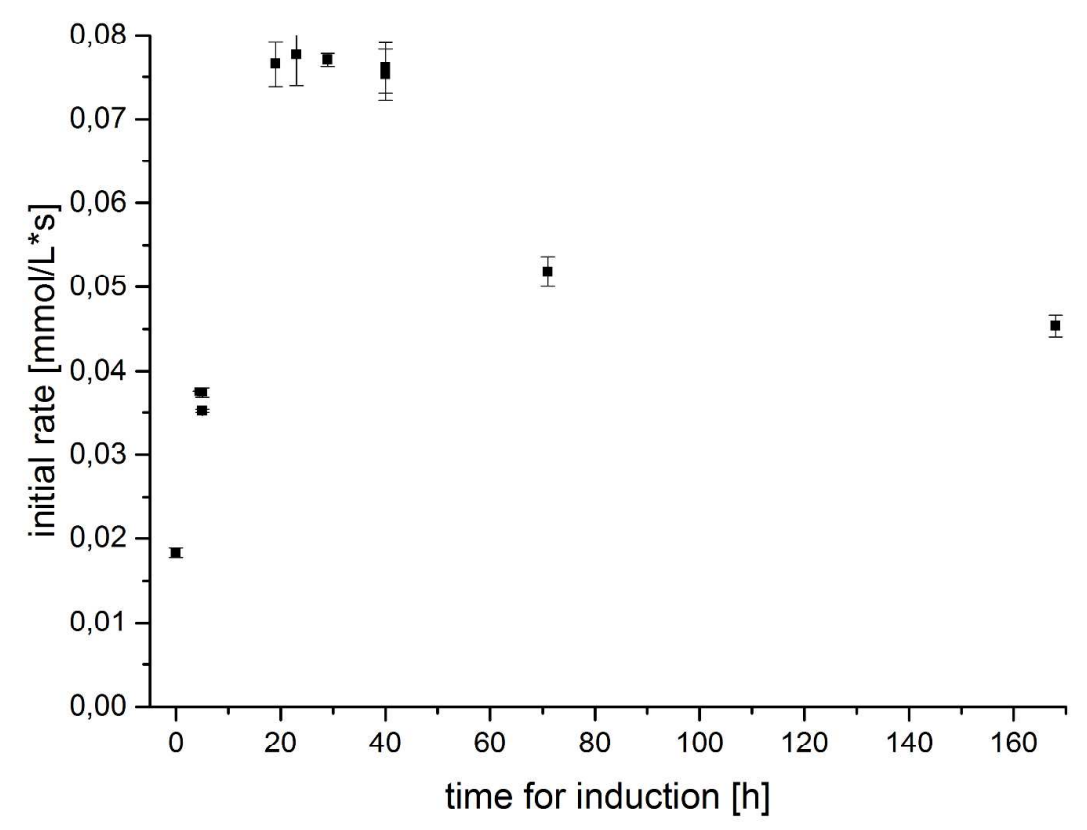

Figure S 5: Maximum reaction rate for the catalytic hydrosilylation using (3) as catalyst with induction ahead depending on the duration of the pretreatment.

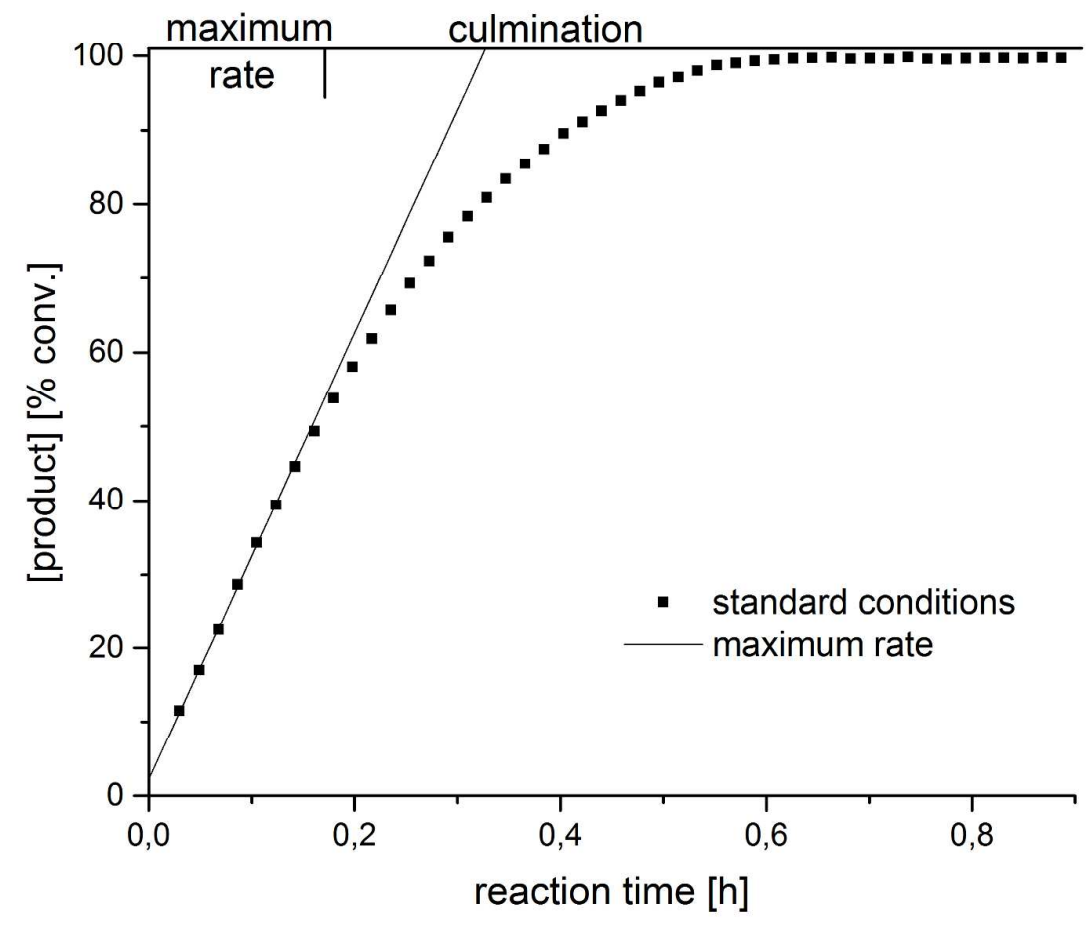

Figure S 6: Typical reaction profile for the catalytic hydrosilylation using (3) as catalyst with induction ahead. 

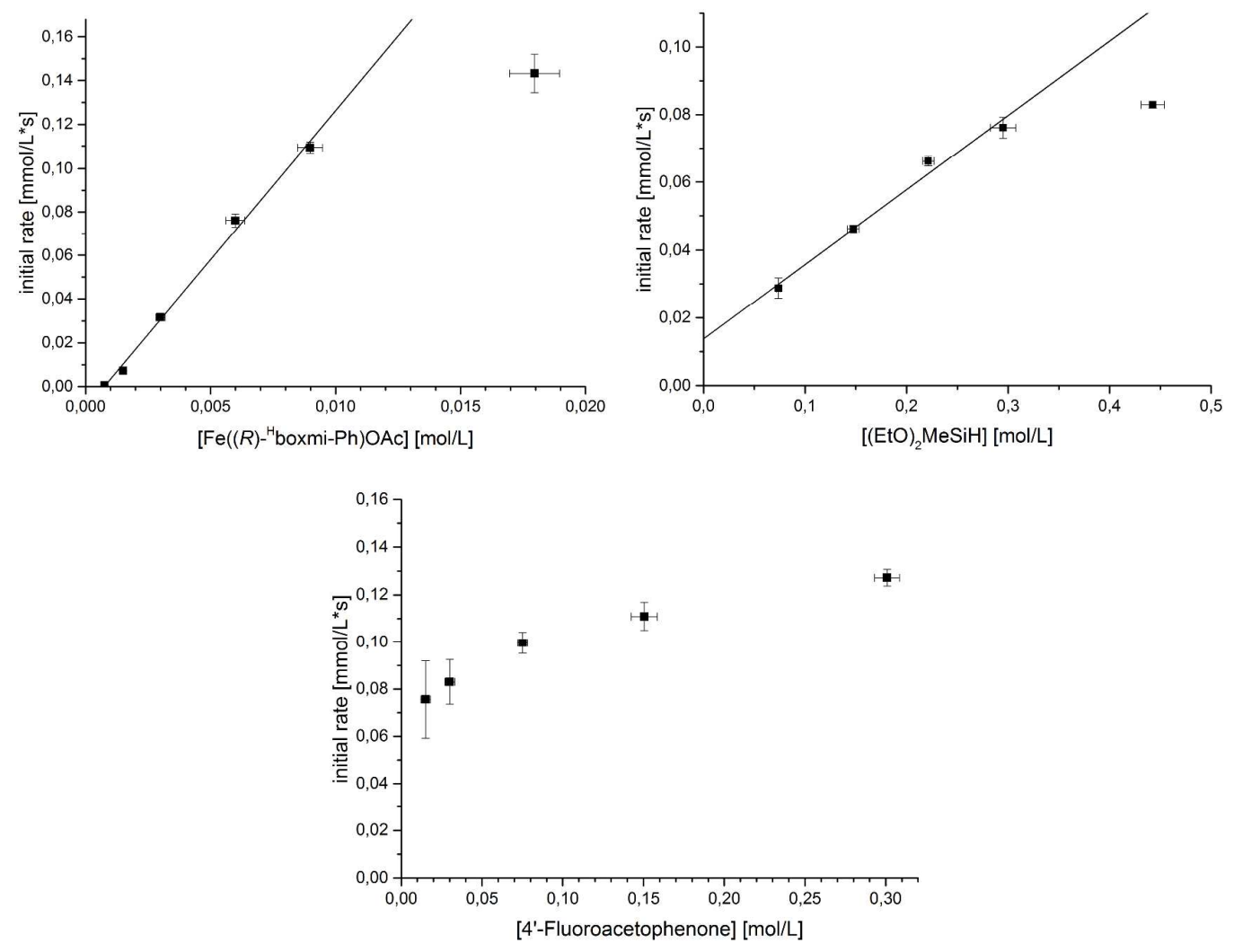

Figure S 7: Determining the rate law for the catalytic hydrosilylation with induction ahead using $\mathrm{Fe}$ (boxmi)OAc (3) as catalyst. The rate law was found to be first order in [catalyst], $\left[(\mathrm{EtO})_{2} \mathrm{MeSiH}\right]$, and [ketone], whereas the latter shows a relatively small slope, that is reminiscent of a saturation behavior. 


\section{Low Temperature Kinetics Using $\mathrm{Fe}($ boxmi)OR* (5) as Catalyst}

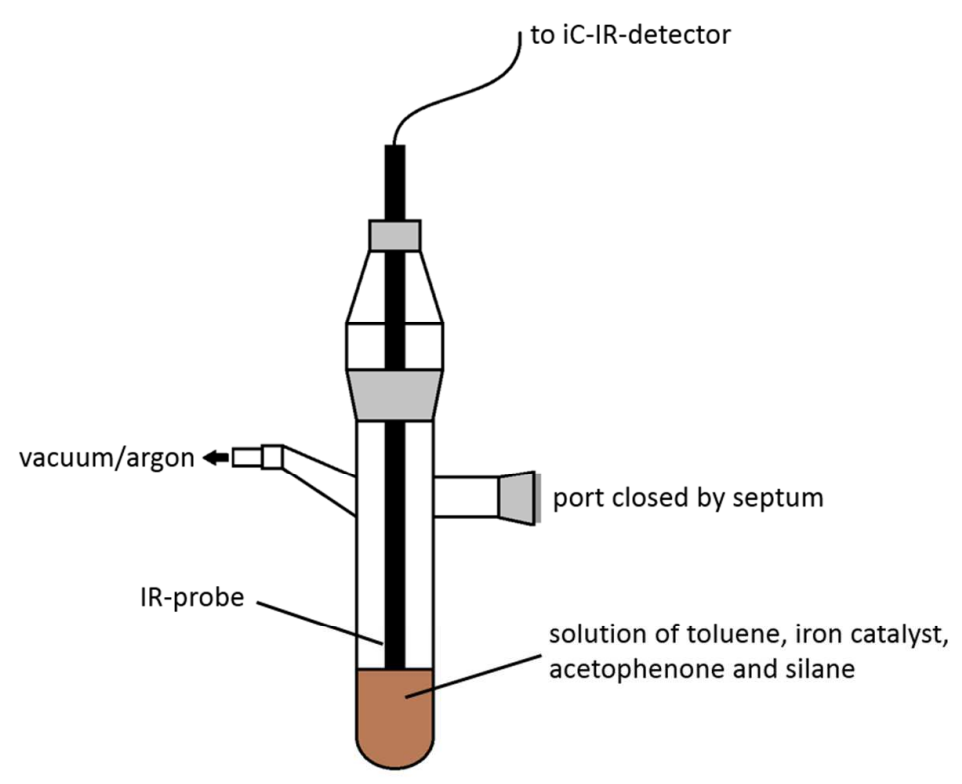

Figure S 8: custom made reaction flask.

A flame-dried reaction flask was equipped with a magnetic stir bar and an IR-probe of the iC-IR-spectrometer (Mettler Toledo ReactIR ${ }^{\mathrm{TM}} 15$ with a SiComp ${ }^{\mathrm{TM}}$ probe head, connected via DST-AgX-fibre optics (9.5 mm diameter)), purged five times with vacuum/argon and cooled to the desired temperature (usually $-40^{\circ} \mathrm{C}$ ) using a cryostat. 3 $\mathrm{ml}$ abs. toluene were added and after thermal equilibration of at least 5 min a background with 512 scans was collected between $1800 \mathrm{~cm}^{-1}$ and $1600 \mathrm{~cm}^{-1}$ at $4 \mathrm{~cm}^{-1}$ intervals. Subsequently the measurement was started: As soon as a steady baseline had been recorded, the iron catalyst was added as a solution in $1 \mathrm{ml}$ abs. toluene and desired amount of acetophenone. From now on, 30 scans were collected every 15 s. Absorption of the $v_{\mathrm{CO}}$ stretching vibration was recorded at $1692 \mathrm{~cm}^{-1}$, the corresponding peak area was used as an indicator for the even distribution of ketone and iron catalyst in solution. As soon as a constant peak area was recorded, but at least after $5 \mathrm{~min}$, $(\mathrm{EtO})_{2} \mathrm{MeSiH}$ was added, resulting in the decrease of the peak at $1692 \mathrm{~cm}^{-1}$. After ending the collection of data, the reaction mixture was then quenched with $2 \mathrm{ml}$ of a saturated solution of potassium carbonate in methanol, allowed to warm to room temperature, filtered over silica and analyzed using chiral HPLC with regard to conver- 
sion and ee.
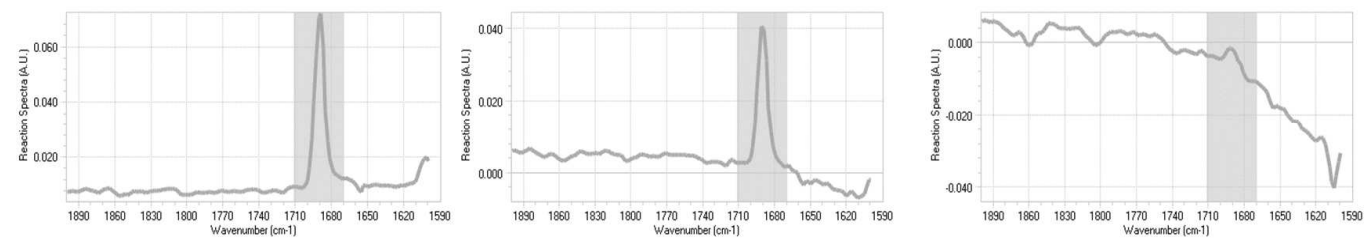

Figure S 9: Typical spectrum at $0 \%, 50 \%$ and full conversion, the area used for analysis in grey.

The area under the peak from $1710 \mathrm{~cm}^{-1}$ to $1670 \mathrm{~cm}^{-1}$ compared to the single point baseline at $1780 \mathrm{~cm}^{-1}$ was used for analysis. The first 5 points after addition of the silane were taken into account for linear regression analysis. The slope values and corresponding errors were used without further adaptation.

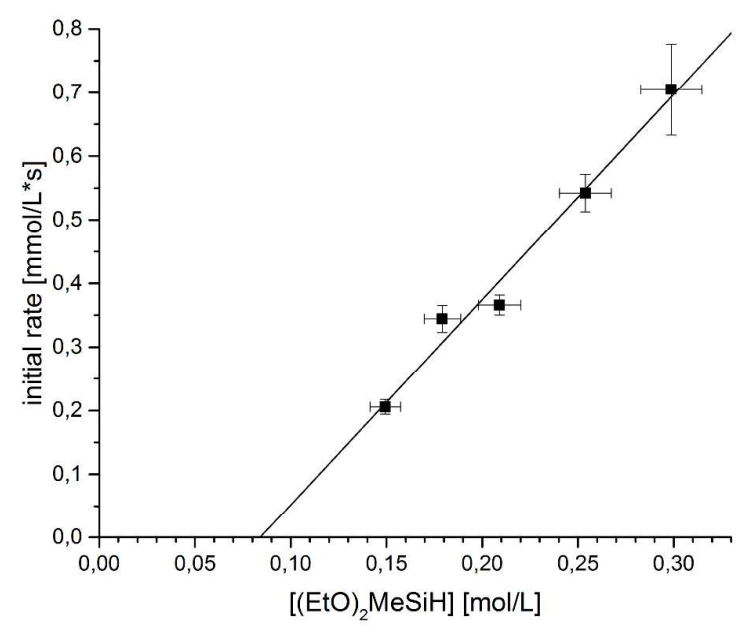

Figure S 10: Initial rate depending on the silane concentration. 


\section{Details for Mechanistic Experiments}

\section{(a) Competition Experiments for Hammett Plots}

General procedure for competition experiments using $\left[\mathrm{Fe}\left((\boldsymbol{R})-{ }^{\mathrm{H}} \mathrm{boxmi}-\mathrm{Ph}\right) \mathrm{OAc}\right]$

\section{(3) as catalyst:}

Iron complex 3 (4 mol\%), (EtO) ${ }_{2} \mathrm{MeSiH}(52 \mu \mathrm{L})$, 1,4-bis(trimethylsilyl)benzene (6.0 $\mathrm{mg}$ ) were dissolved in toluene- $\mathrm{d}_{8}$ (total volume $535 \mu \mathrm{L}$ ) in a J. Young NMR tube. Experiments with preactivated iron complex were heated to $65^{\circ} \mathrm{C}$ for $20 \mathrm{~h}$. $4^{\prime}$ substituted acetophenone derivatives $(6-8 \mathrm{mg}$ each) were added at room temperature. The reaction mixture was heated to $65{ }^{\circ} \mathrm{C}$ in the NMR-spectrometer and ${ }^{1} \mathrm{H}$ - and ${ }^{13} \mathrm{C}$ NMR spectra were recorded.

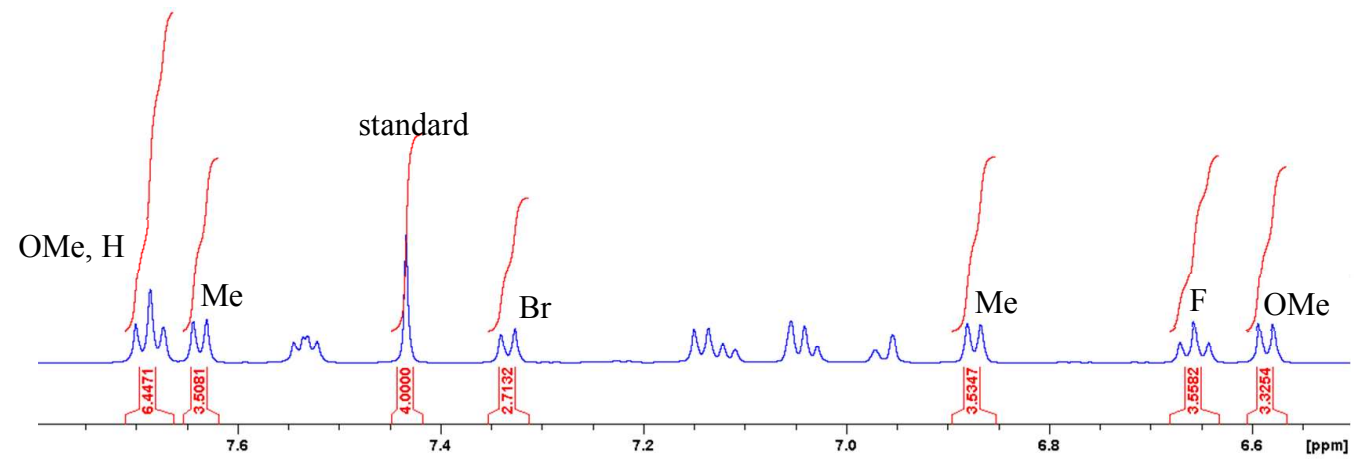

Figure S 11: Exemplary ${ }^{1} \mathrm{H}-\mathrm{NMR}$ spectrum of the competition hydrosilylation of acetophenone derivatives $(\mathrm{R}=\mathrm{Br}, \mathrm{F}, \mathrm{H}, \mathrm{Me}, \mathrm{OMe})$ carried out at $338 \mathrm{~K}$ in toluene- $\mathrm{d}_{8}$.

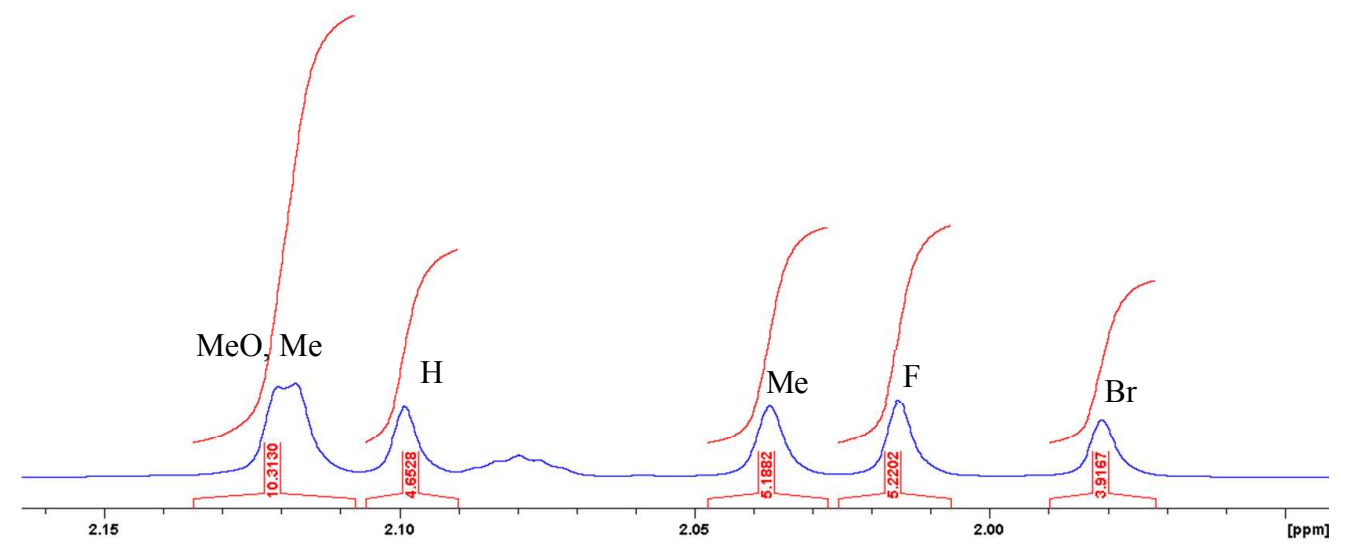

Figure S 12: Exemplary ${ }^{1} \mathrm{H}-\mathrm{NMR}$ spectrum of the competition hydrosilylation of acetophenone derivatives $(\mathrm{R}=\mathrm{Br}, \mathrm{F}, \mathrm{H}, \mathrm{Me}, \mathrm{OMe})$ carried out at $338 \mathrm{~K}$ in toluene- $\mathrm{d}_{8}$. 


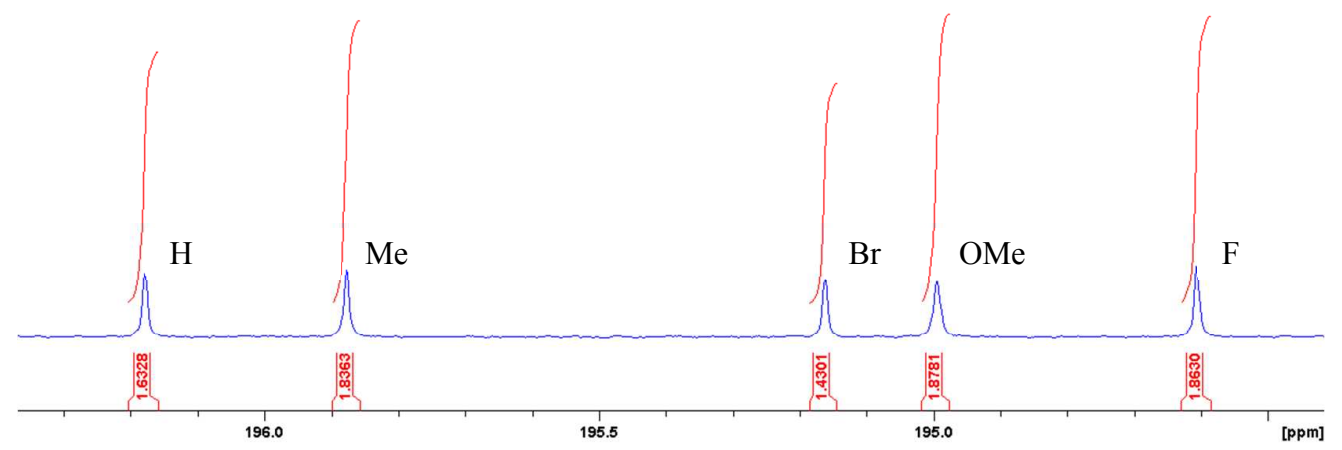

Figure S 13: Exemplary ${ }^{13} \mathrm{C}-\mathrm{NMR}$ spectrum of the competition hydrosilylation of acetophenone derivatives $(\mathrm{R}=\mathrm{Br}, \mathrm{F}, \mathrm{H}, \mathrm{Me}, \mathrm{OMe})$ carried out at $338 \mathrm{~K}$ in toluene- $\mathrm{d}_{8}$.

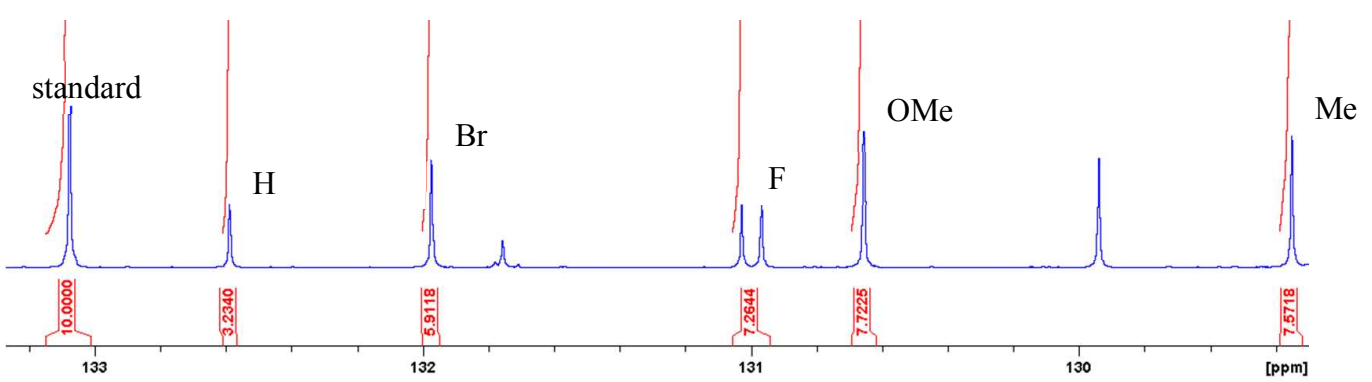

Figure S 14: Exemplary ${ }^{13} \mathrm{C}-\mathrm{NMR}$ spectrum of the competition hydrosilylation of acetophenone derivatives $(\mathrm{R}=\mathrm{Br}, \mathrm{F}, \mathrm{H}, \mathrm{Me}, \mathrm{OMe})$ carried out at $338 \mathrm{~K}$ in toluene- $\mathrm{d}_{8}$.

Using the following equation, relative rate constants were determined from the NMR integrals. $^{8}$

$$
\frac{k_{x}}{k_{H}}=\frac{\ln \left(\frac{c_{x, t}}{c_{x, t=0}}\right)}{\ln \left(\frac{c_{H, t}}{c_{H, t=0}}\right)}
$$

Overlapping signals were not taken into account. It did not make a significant difference, whether all four signals of each compound or only the ${ }^{13} \mathrm{C}$-signal in the aromatic region was used for calculation of the relative rates. The error for the NMR-integrals is assumed to be $5 \%, \sigma$-values and the respective errors were taken from literature. ${ }^{9,10}$ According to equation (2), the relative rates were plotted against the $\sigma$-coefficient.

$$
\log _{10}\left(\frac{k_{x}}{k_{H}}\right)=\rho \cdot \sigma
$$




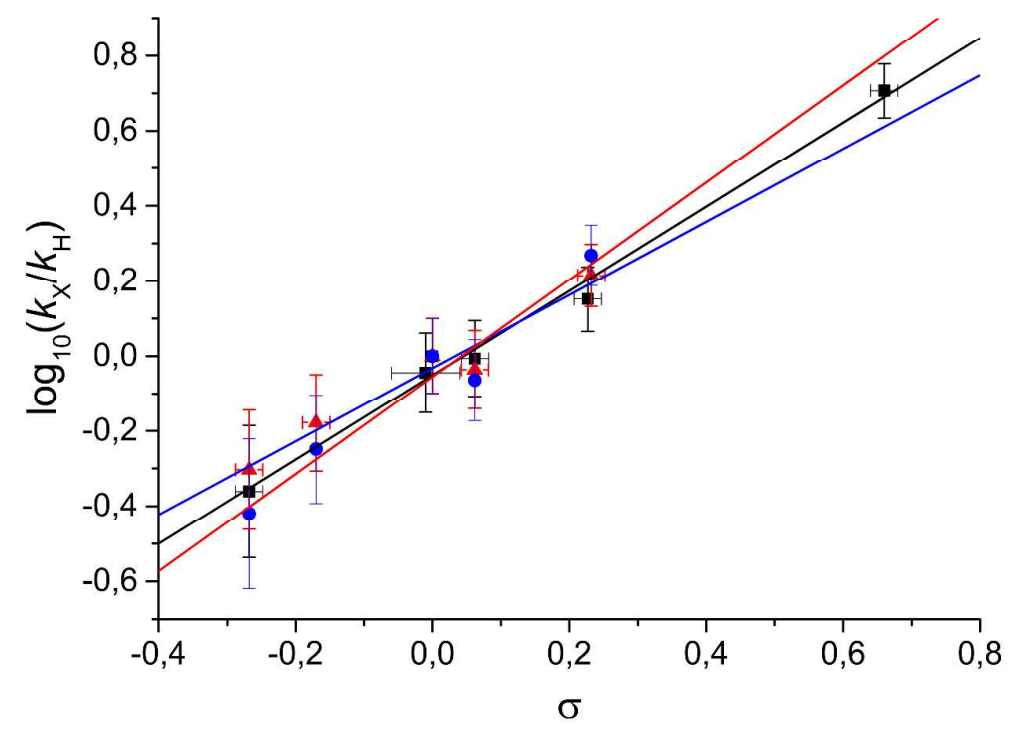

Figure S 15: Hammett plot for the competitive hydrosilylation of acetophenone derivatives $(\mathrm{R}=\mathrm{Br}, \mathrm{Cl}, \mathrm{CN}, \mathrm{F}, \mathrm{H}, \mathrm{Me}, \mathrm{Ph}, \mathrm{OMe})$ carried out at $338 \mathrm{~K}$ in toluene- $\mathrm{d}_{8}$, analyzed using ${ }^{1} \mathrm{H}$ and ${ }^{13} \mathrm{C}$ NMR-spectroscopy (red and blue) or ${ }^{13} \mathrm{C}-\mathrm{NMR}$-spectroscopy only (black). The samples for the red and the black datapoints were activated prior to addition of the ketone.

Table S 1: $\rho$-values derived from Hammett plots for hydrosilylation of 4'-substituted acetophenones.

\begin{tabular}{lllll}
\hline entry & color $^{\mathrm{a}}$ & $\rho$ & $\mathrm{R}^{\mathrm{b}}$ & comment \\
\hline 1 & red & $0.97 \pm 0.12$ & $\mathrm{Br}, \mathrm{F}, \mathrm{H}, \mathrm{Me}, \mathrm{OMe}$ & activated \\
2 & blue & $1.29 \pm 0.17$ & $\mathrm{Br}, \mathrm{F}, \mathrm{H}, \mathrm{Me}, \mathrm{OMe}$ & No preactivation \\
3 & black & $1.12 \pm 0.06$ & $\mathrm{Cl}, \mathrm{CN}, \mathrm{F}, \mathrm{H}, \mathrm{Ph}, \mathrm{OMe}$ & $\begin{array}{l}\text { activated, } \\
\end{array}$ \\
& & & ${ }^{13} \mathrm{C}$ aromatic signal only \\
\hline
\end{tabular}

${ }^{\mathrm{a}}$ referring to Figure $\mathrm{S} 15 .{ }^{\mathrm{b}} 4$ '-substituents within a competitive reaction

\section{General procedure for competition experiments using $\left[\mathrm{Fe}\left((\mathrm{R})-{ }^{\mathrm{H}} \mathrm{boxmi}-\right.\right.$ $\mathrm{Ph}) \mathrm{OCHCH}_{3} \mathrm{Ph}$ (5) as catalyst:}

Iron complex 5 (7.8 mg, $13 \mu \mathrm{mol})$, 1,3,5-tri(methoxy)benzene (14.0 mg, $83 \mu \mathrm{mol})$, and the ketones (20-35 mg each, 140-180 $\mu \mathrm{mol}$ each) were dissolved in $0.7 \mathrm{~mL}$ toluene- $\mathrm{d}_{8}$ in a J. Young NMR tube. A reference spectrum was measured. The solution was transferred to a vial, cooled to $-40{ }^{\circ} \mathrm{C}$ and diethoxymethylsilane $(10 \mu \mathrm{L}, 62 \mu \mathrm{mol})$ was added at low temperature. $\mathrm{A}{ }^{13} \mathrm{C}$ NMR spectrum of this solution was recorded. The procedure to add silane was repeated thrice and all three data points were taken 
into account for the analysis. The values given in the main paper are an average of three independent runs.
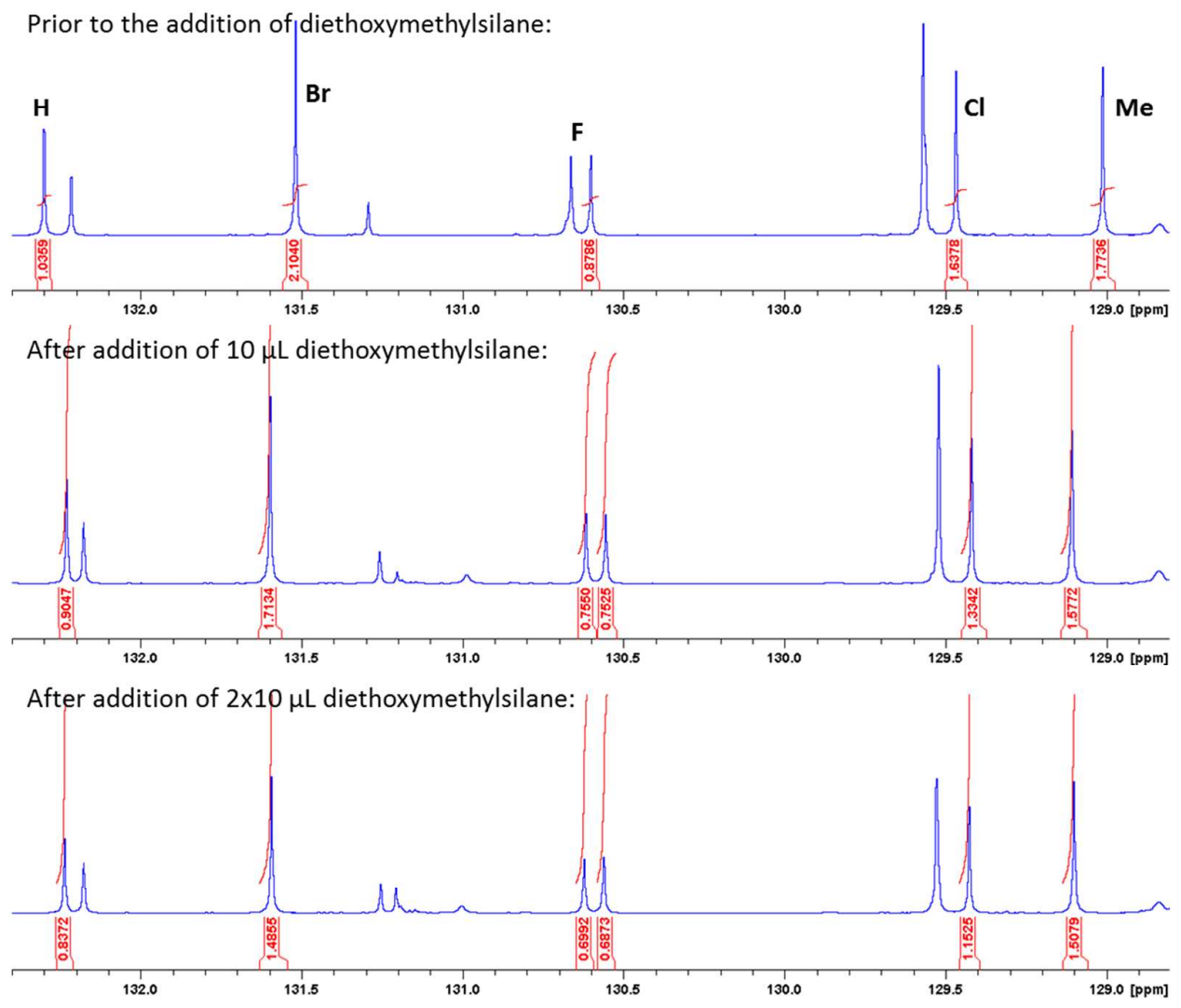

Figure S 16: Exemplary ${ }^{13} \mathrm{C}-\mathrm{NMR}$ spectra of the competition hydrosilylation of acetophenone derivatives $(\mathrm{R}=\mathrm{Br}, \mathrm{Cl}, \mathrm{F}, \mathrm{H}, \mathrm{Me}, \mathrm{OMe})$ carried out at $298 \mathrm{~K}$ in toluene- $\mathrm{d}_{8}$. The signal at $162.16 \mathrm{ppm}$ of 1,3,5-tri(methoxy)benzene was taken as internal standard and its integral was set to 1. For 4'-Methoxyacetophenone, the signal at $113.76 \mathrm{ppm}$ was used.

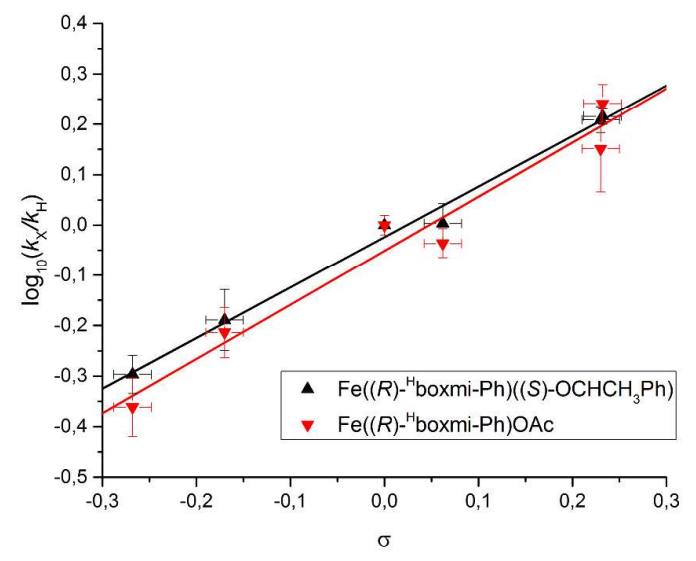

Figure S 17: Hammett plot for both precatalysts. 


\section{(b) Determination of the ${ }^{1} \mathrm{H}-{ }^{2} \mathrm{H}$ Kinetic Isotope Effect Using Competition Ex- periments}

Iron complex 5 (2.6 mg, $4.3 \mu \mathrm{mol})$ and 4'-phenyl-acetophenone (25.0 mg, $127 \mu \mathrm{mol})$ were dissolved in $1.0 \mathrm{~mL}$ abs. toluene and cooled to $-78^{\circ} \mathrm{C}$. A mixture of $40 \mu \mathrm{L}$ (EtO) $)_{2} \mathrm{MeSiH}(250 \mu \mathrm{mol}), 40 \mu \mathrm{L}(\mathrm{EtO})_{2} \mathrm{MeSiD}(250 \mu \mathrm{mol})$ and $100 \mu \mathrm{L}$ toluene was added dropwise. The mixture was allowed to warm to room temperature in the cold bath within $5 \mathrm{~h}$. After stirring $1 \mathrm{~h}$ at room temperature, $0.5 \mathrm{~mL}$ of a saturated $\mathrm{K}_{2} \mathrm{CO}_{3}$ solution in $\mathrm{MeOH}$ were added. Filtration through a plug of silica and subsequent column chromatography yielded the desired product. The ratio of deuterated to normal product was determined by ${ }^{13} \mathrm{C}$ NMR spectroscopy and averaged over two independent runs.

With 3 as catalyst: Iron complex 3 (2.3 mg, $4 \mu \mathrm{mol})$, 4'-fluoroacetophenone (10 $\mu \mathrm{L}$, $0.082 \mathrm{mmol})$, and the two silanes $(13 \mu \mathrm{L}$ each, $0.081 \mathrm{mmol}$ each) were dissolved in $0.5 \mathrm{~mL}$ toluene- $\mathrm{d}_{8}$. The mixture was heated to $60{ }^{\circ} \mathrm{C}$ for $24 \mathrm{~h}$ and quenched with 0.5 $\mathrm{mL} \mathrm{K}_{2} \mathrm{CO}_{3} / \mathrm{MeOH}$. Filtration through a plug of silica and subsequent column chromatography yielded the desired product. The ratio of deuterated to normal product was determined by ${ }^{13} \mathrm{C}$ NMR spectroscopy and averaged over two independent runs.

For the run with induction ahead only one change was made, 3 was heated with the mixture of silanes for $20 \mathrm{~h}$ prior to the addition of the ketone. 


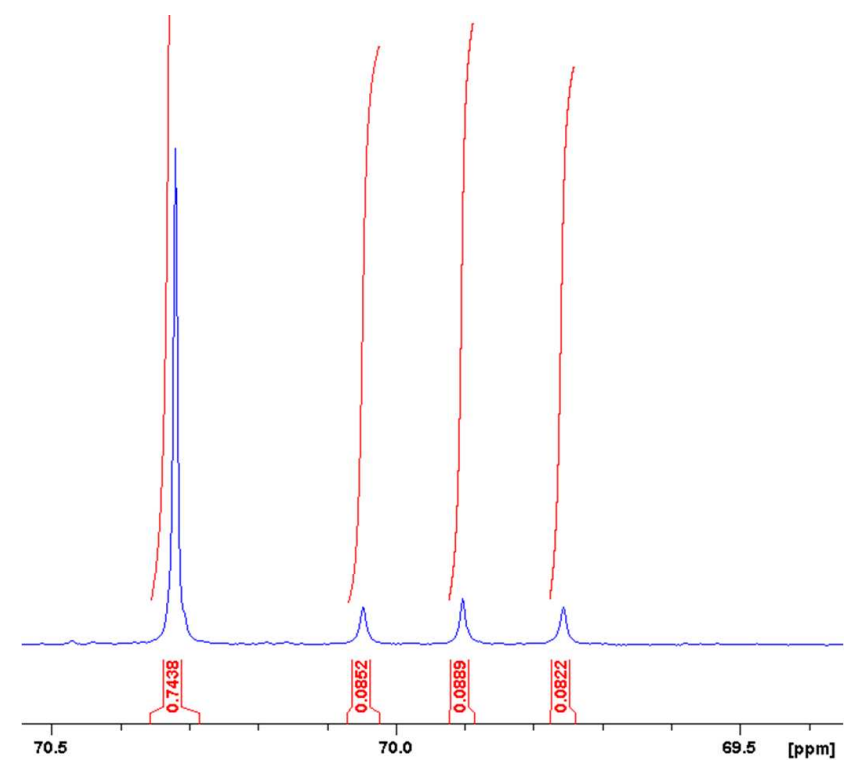

Figure S 18: Exemplary ${ }^{13} \mathrm{C}-\mathrm{NMR}$ spectrum of the competition hydrosilylation of 4'phenyl-acetophenone with (EtO) $2 \mathrm{MeSiH}$ and (EtO)2MeSiD.

\section{(c) Mercury Test}

To a solution of $5(5.2 \mathrm{mg}, 8.5 \mu \mathrm{mol})$ and acetophenone $(15 \mu \mathrm{L}, 130 \mu \mathrm{mol})$ in $2 \mathrm{~mL}$ toluene, $\mathrm{Hg}^{0}$ (325 mg, $1.6 \mathrm{mmol}$ ) was added and the emulsion was stirred vigorously for $15 \mathrm{~min}$ at room temperature. (EtO) $)_{2} \mathrm{MeSiH}(42 \mu \mathrm{L}, 260 \mu \mathrm{mol})$ was added and the reaction quenched with $0.5 \mathrm{~mL}$ of a saturated solution of $\mathrm{K}_{2} \mathrm{CO}_{3}$ in $\mathrm{MeOH}$ after 30 min. Filtration through Celite and subsequent HPLC analysis yielded full conversion and $95 \%$ ee.

A solution of $3(14.7 \mathrm{mg}, 27 \mu \mathrm{mol})$ and $(\mathrm{EtO})_{2} \mathrm{MeSiH}(42 \mu \mathrm{L}, 260 \mu \mathrm{mol})$ in $2 \mathrm{~mL}$ toluene was heated to $65^{\circ} \mathrm{C}$ for $16 \mathrm{~h}$. $\mathrm{Hg}^{0}(232 \mathrm{mg}, 1.2 \mathrm{mmol})$ was added and the emulsion was stirred vigorously for $5 \mathrm{~min}$. Afterwards, acetophenone $(15 \mu \mathrm{L}, 130 \mu \mathrm{mol})$ was added and the reaction mixture was heated to $65^{\circ} \mathrm{C}$ for $46 \mathrm{~h}$. Quenching with $0.5 \mathrm{~mL} \mathrm{~K}_{2} \mathrm{CO}_{3} / \mathrm{MeOH}$ and filtration through Celite yielded the same conversion and selectivity as a sample without addition of $\mathrm{Hg}^{0}$. 


\section{(d) ee vs. $T$}

$5(2.0 \mathrm{mg}, 3.4 \mu \mathrm{mol})$ and 4'-phenyl-acetophenone $(10.1 \mathrm{mg}, 51 \mu \mathrm{mol})$ in $1.0 \mathrm{~mL}$ toluene were added to a schlenk flask and equilibrated to the given temperature for 15 min. (EtO) $)_{2} \mathrm{MeSiH}(20 \mu \mathrm{L}, 125 \mu \mathrm{mol})$ was added dropwise. After the time given in

Table $\mathrm{S} 2$, the reaction was quenched using $0.5 \mathrm{~mL}$ saturated $\mathrm{K}_{2} \mathrm{CO}_{3}$ in $\mathrm{MeOH}$ and allowed to warm/cool to room temperature. The mixture was filtered through silica and then analyzed using HPLC. All reported values are an average of at least two independent runs.

Table S 2: reaction conditions for ee vs. T plot.

\begin{tabular}{lllll}
\hline entry & $\mathbf{T} / \mathbf{K}$ & $\mathbf{\Delta T} / \mathbf{K}$ & $\mathbf{t} / \mathbf{h}$ & ee / \% \\
\hline 1 & 338.2 & 1.0 & 0.25 & 92.0 \\
2 & 313.7 & 1.0 & 0.25 & 94.0 \\
3 & 298.9 & 0.5 & 0.5 & 95.2 \\
4 & 273.2 & 0.2 & 1.0 & 97.3 \\
5 & 253.2 & 0.2 & 2.0 & 97.4 \\
6 & 233.2 & 0.2 & 4.5 & 98.1 \\
\hline
\end{tabular}

(e) ee vs. conv.

To a solution of $5(2.5 \mathrm{mg}, 4.1 \mu \mathrm{mol})$ and acetophenone $(10 \mu \mathrm{L}, 86 \mu \mathrm{mol})$ in $0.5 \mathrm{~mL}$ toluene (EtO) $)_{2} \mathrm{MeSiH}$ (2.7 to $24 \mu \mathrm{L}, 17$ to $150 \mu \mathrm{mol}$, six different samples) was added dropwise at room temperature. After $20 \mathrm{~min}$, the reaction was quenched using $0.5 \mathrm{~mL}$ saturated $\mathrm{K}_{2} \mathrm{CO}_{3}$ in $\mathrm{MeOH}$. The mixture was filtered through silica and then analyzed using HPLC.

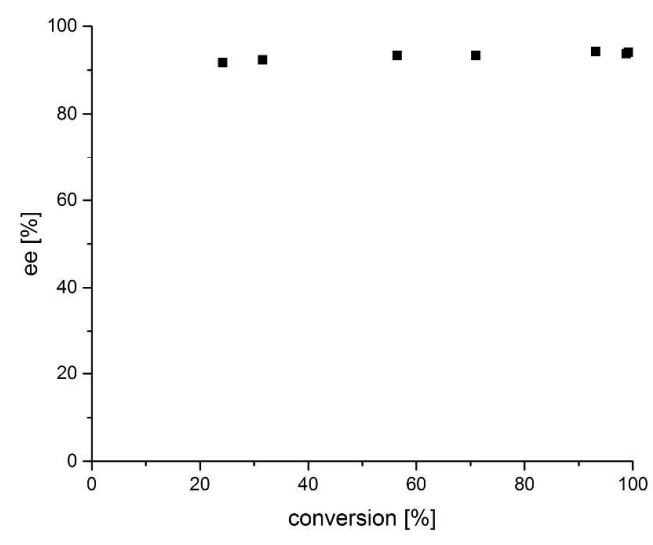

Figure S 19: Measurement of the ee depending on the conversion. 


\section{(f) Radical Clock}

The radical clock $6^{6}$ as well as the corresponding cyclopropyl carbinol $7^{7}$ were synthesized according to literature procedures.

A solution of $5(2.9 \mathrm{mg}, 4.8 \mu \mathrm{mol})$ and the radical clock $6(19 \mu \mathrm{L}, 85 \mu \mathrm{mol})$ in $1 \mathrm{~mL}$ toluene was cooled to $-78^{\circ} \mathrm{C}$. (EtO) $)_{2} \mathrm{MeSiH}(30 \mu \mathrm{L}, 190 \mu \mathrm{mol})$ was added and the reaction was allowed to stir over night. The reaction was quenched with $0.5 \mathrm{~mL}$ of a saturated solution of $\mathrm{K}_{2} \mathrm{CO}_{3}$ in $\mathrm{MeOH}$. Filtration through silica and subsequent HPLC analysis (Macherey-Nagel Nucleodur 100-5 column, ${ }^{n}$ hexane $99.5 /{ }^{i} \mathrm{PrOH} 0.5,1.0$ $\mathrm{mL} / \mathrm{min}, 20^{\circ} \mathrm{C}, 210 \mathrm{~nm}$ ) yielded $79 \%$ of the cyclopropyl carbinol $7,19 \%$ of the starting ketone 7 , and $2 \%$ of the open form 8 .

Workup with $\mathrm{K}_{2} \mathrm{CO}_{3}$ in MeOD- $\mathrm{d}_{4}$ and analyzing using ${ }^{2} \mathrm{H}$ NMR spectroscopy confirmed the presence of less than $5 \%$ of the open form 8 .

The same results were found using $\mathrm{Fe}\left(\right.$ boxmi)OAc (3) as catalyst at $65^{\circ} \mathrm{C}$.

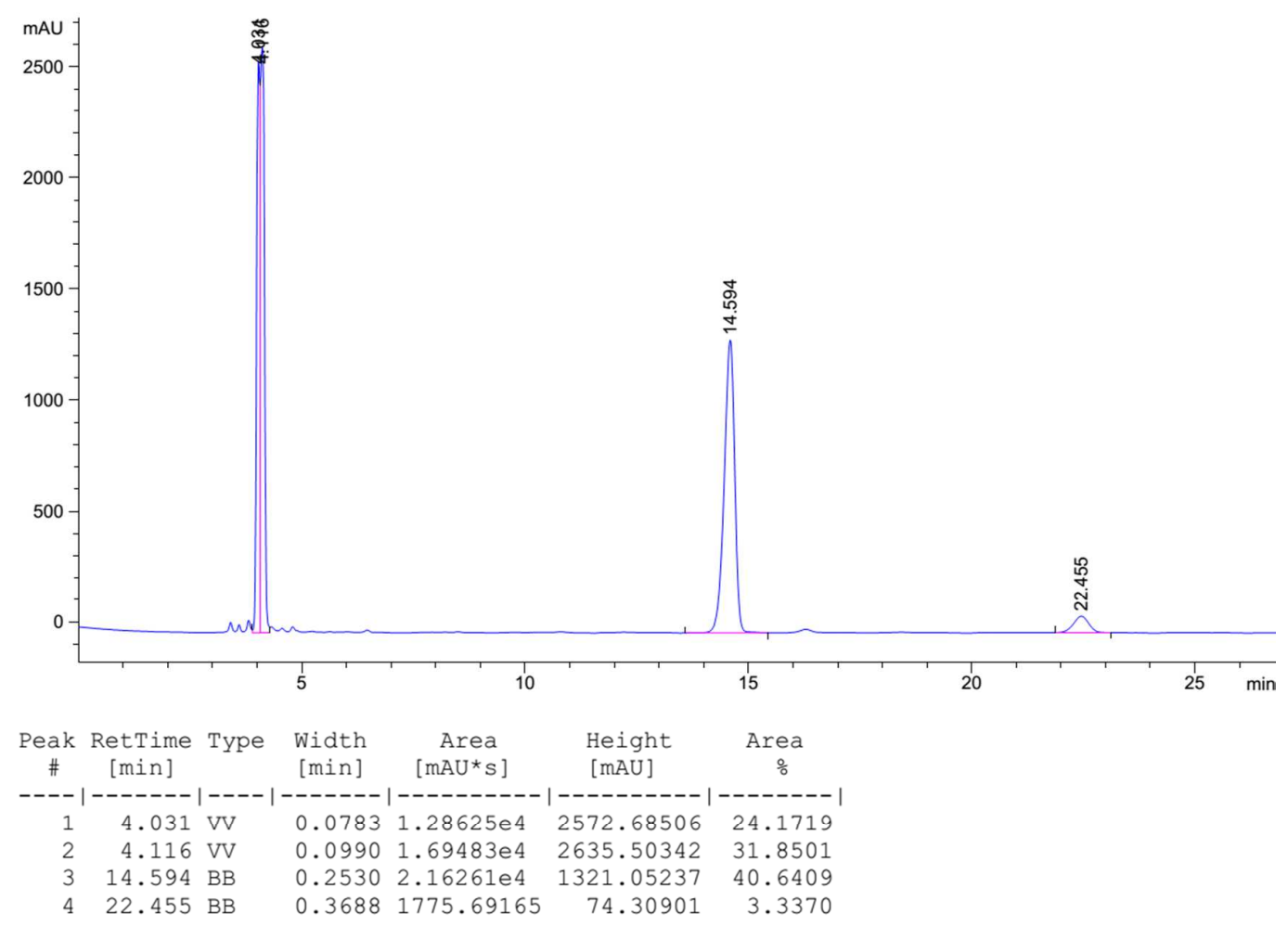

Figure S 20: HPLC trace of the reference substances dissolved in hexane. The open form of the radical clock 8 has a $t_{R}$ of 4.0 min, the starting material 6 has a $t_{R}$ of 4.1 
$\min$, the diastereomers of the product alcohol 7 have retention times of $t_{R 1}=14.6 \mathrm{~min}$ and $t_{\mathrm{R} 2}=22.5 \mathrm{~min}$.

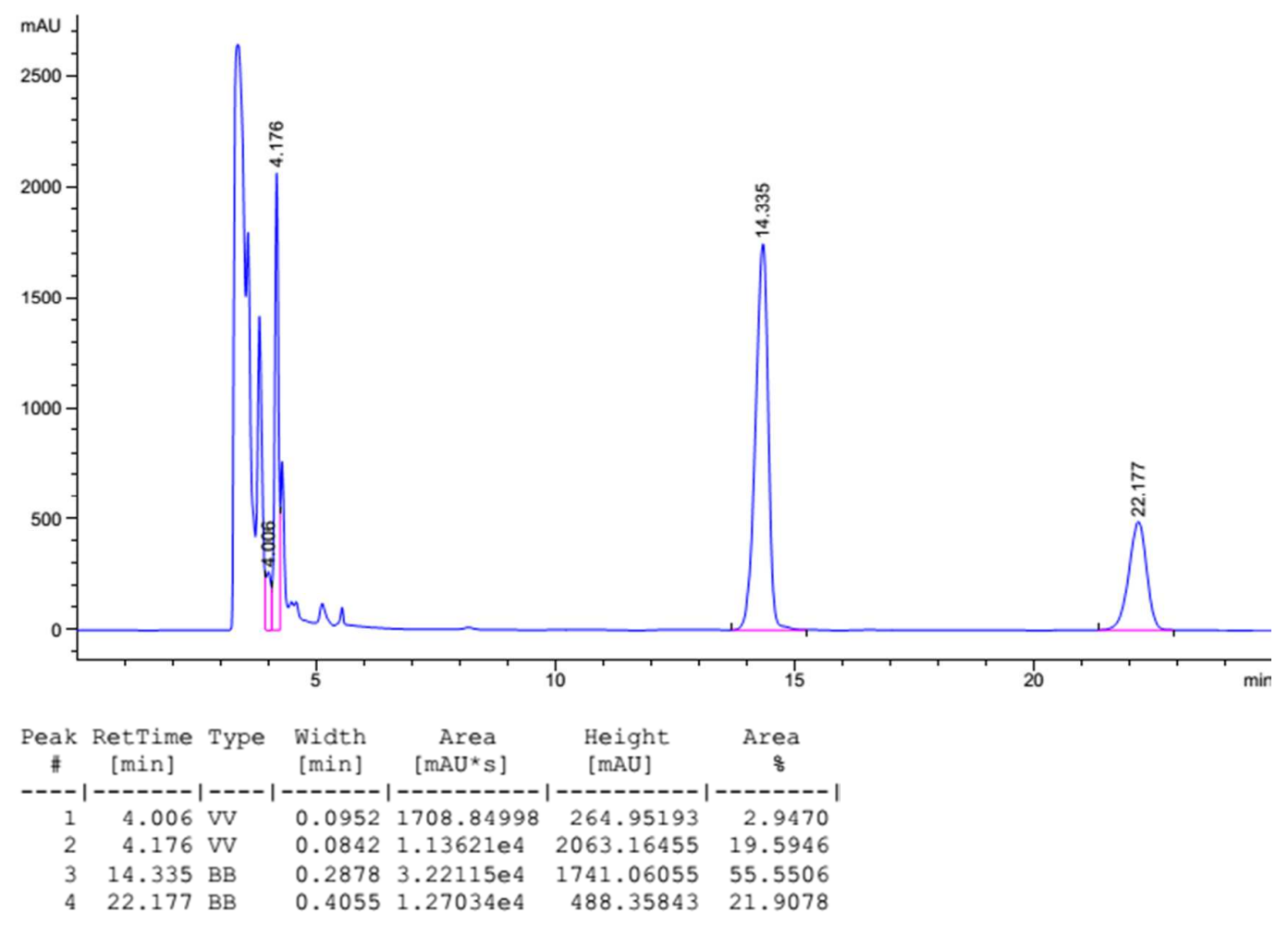

Figure S 21: HPLC trace of the reaction mixture after filtration.

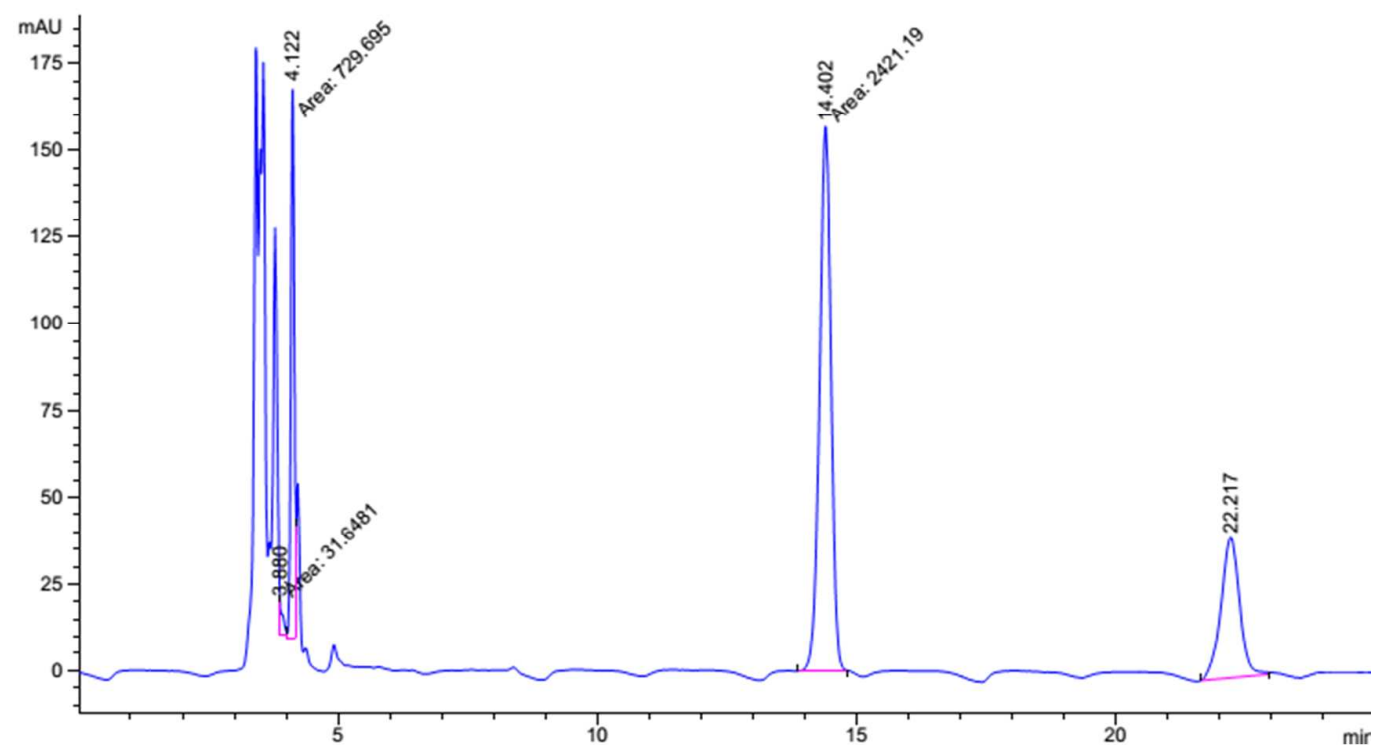




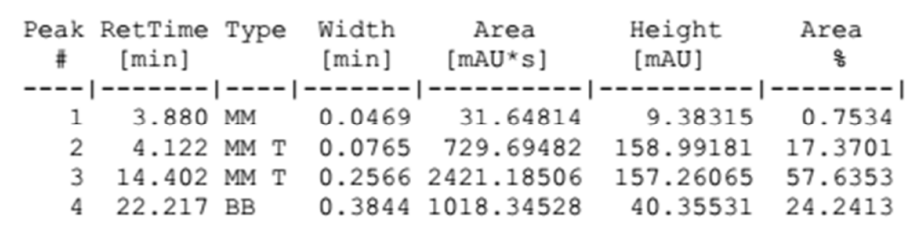

Figure S 22: HPLC trace of the reaction mixture after filtration, evaporation and dissolving in hexane. The retention time for the open form of the radical clock is displayed incorrectly in the table below the chromatogram and should read as $4.0 \mathrm{~min}$.

\section{(g) In Situ NMR Experiments for the Induction Period}

The desired acetate complex $\mathbf{3}, \mathbf{3}-\mathbf{D}_{\mathbf{3}}$, or $\mathbf{3 -}{ }^{\mathbf{1 3}} \mathbf{C}(10-15 \mathrm{mg}, 22 \mu \mathrm{mol})$ was dissolved in $0.5 \mathrm{~mL}$ toluene or toluene- $\mathrm{d}_{8}$ (depending on the experiment) in a J. Young NMR tube. Then $(\mathrm{EtO})_{2} \mathrm{MeSiH}(26-50 \mu \mathrm{L}, 162-312 \mu \mathrm{mol})$ was added and the sample was heated in a NMR spectrometer to $65^{\circ} \mathrm{C}$ while the measurements take place. For each nucleus, at least two separate runs were performed to confirm the results.

One sample was heated externally and taken to the NMR at various times to perform ${ }^{29} \mathrm{Si}-\mathrm{NMR}$ measurements as well as Evans measurements ${ }^{11-13}$ to determine the magnetic susceptibility of the sample. The latter did not show any significant change in the magnetic susceptibility of the sample.

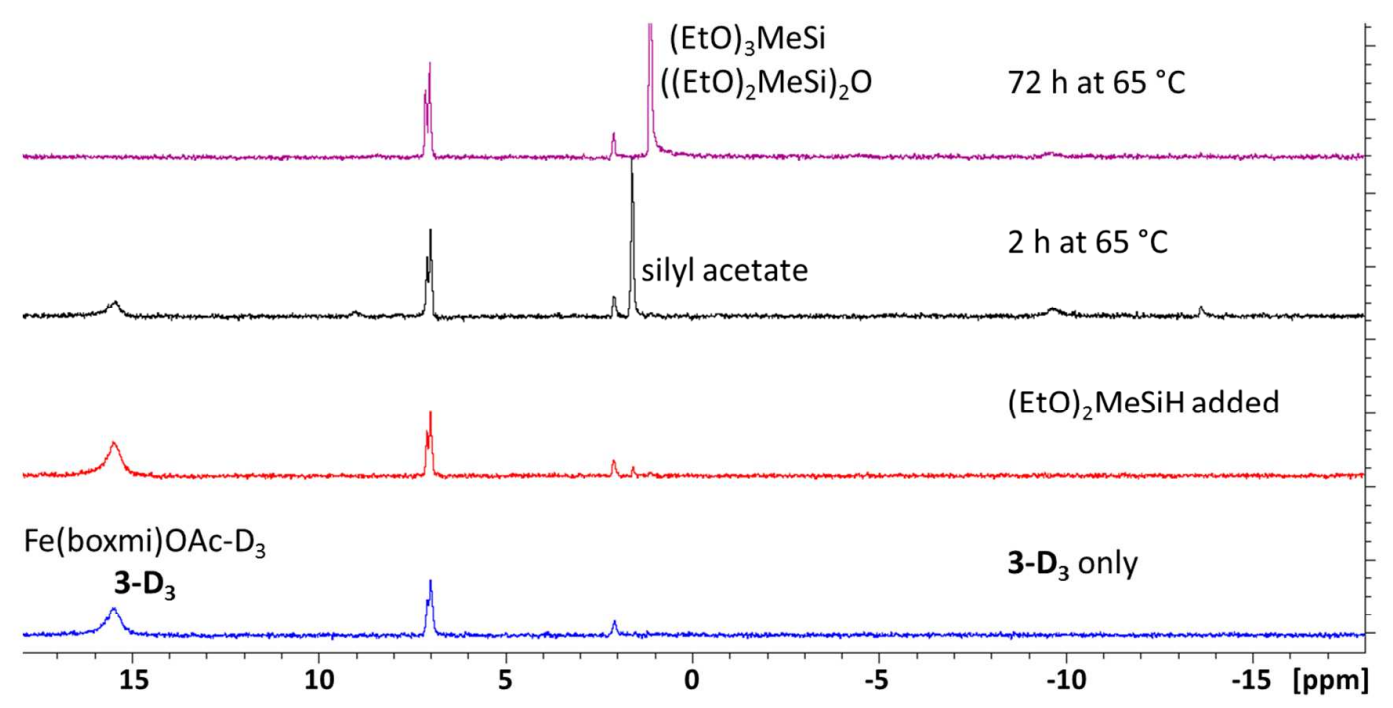

Figure S 23: ${ }^{2} \mathrm{H}$ NMR spectra of the reaction of $\mathbf{3}-\mathbf{D}_{\mathbf{3}}$ with $(\mathrm{EtO})_{2} \mathrm{MeSiH}$ in toluene. 


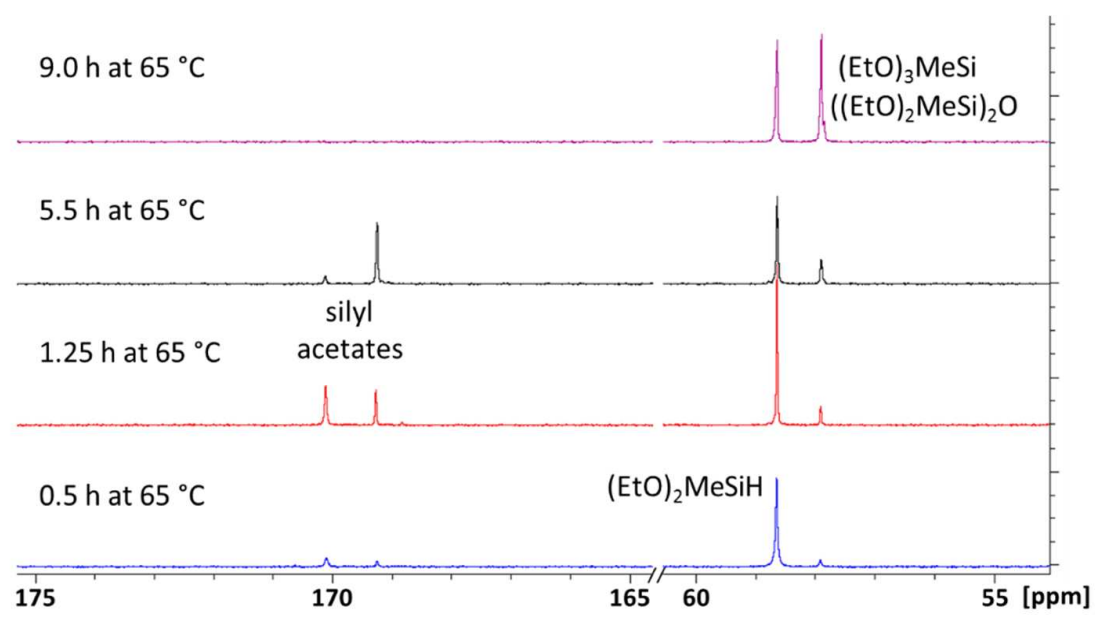

Figure S 24: ${ }^{13} \mathrm{C}$ NMR spectra of the reaction of $3-{ }^{13} \mathbf{C}$ with $(\mathrm{EtO})_{2} \mathrm{MeSiH}$ in toluene.

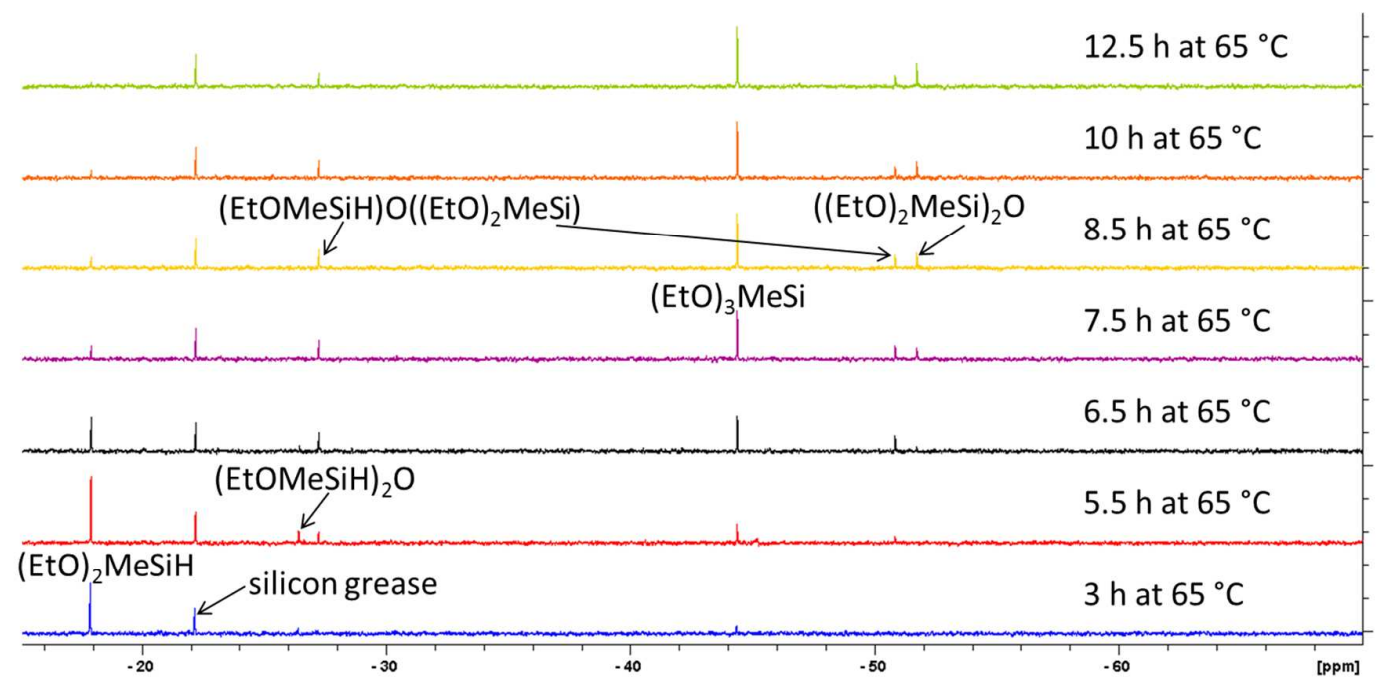

Figure S 25: ${ }^{29} \mathrm{Si}$ dept NMR spectra $\left({ }^{2} J_{\mathrm{SiH}} \approx 7 \mathrm{~Hz}\right)$ of the reaction of $\mathbf{3}$ with $(\mathrm{EtO})_{2} \mathrm{MeSiH}$ in toluene- $\mathrm{d}_{8}$. 


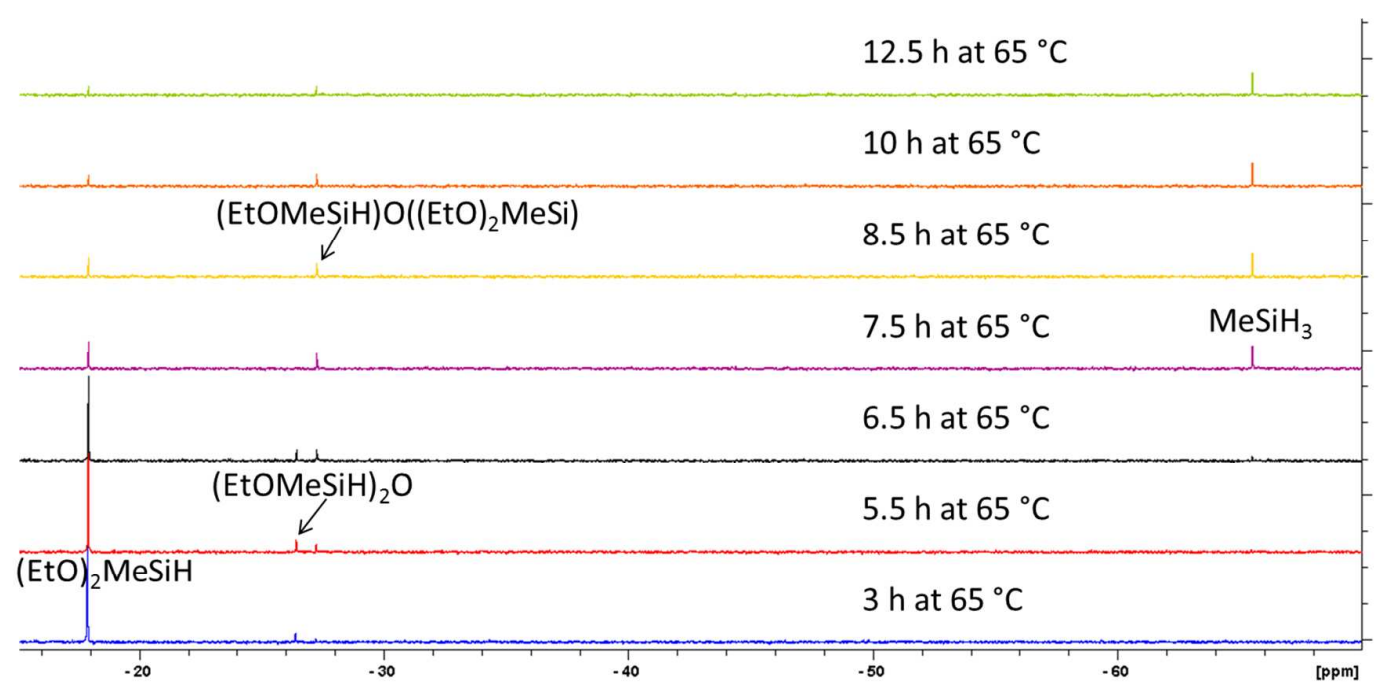

Figure S 26: ${ }^{29} \mathrm{Si}$ dept NMR spectra $\left({ }^{1} J_{\mathrm{SiH}} \approx 200 \mathrm{~Hz}\right)$ of the reaction of $\mathbf{3}$ with $(\mathrm{EtO})_{2} \mathrm{MeSiH}$ in toluene- $\mathrm{d}_{8}$.

The formation of $\mathrm{MeSiH}_{3}$ is attributed to a base-catalyzed silane-scrambling leading to a decomposition of $(\mathrm{EtO})_{2} \mathrm{MeSiH}$ to $(\mathrm{EtO})_{3} \mathrm{MeSi}$ and $\mathrm{MeSiH}_{3}$. This reaction also readily occurs upon adding $\mathrm{NaO}^{t} \mathrm{Bu}$ to a solution of $(\mathrm{EtO})_{2} \mathrm{MeSiH}$ in toluene at room temperature.

\section{(h) In Situ Reaction Sequence to Trace an Iron Hydride Species}

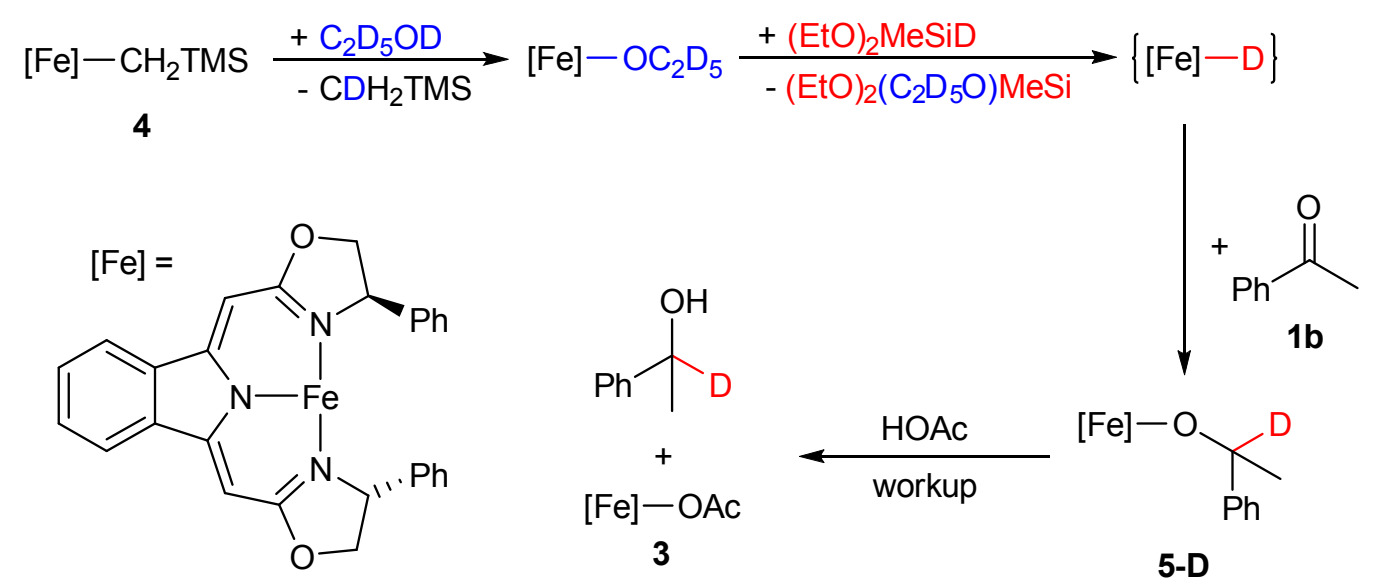

$4(28.9 \mathrm{mg}, 50 \mu \mathrm{mol})$ was dissolved in $0.5 \mathrm{~mL}$ abs. toluene and $\mathrm{C}_{2} \mathrm{D}_{5} \mathrm{OD}(1.3 \mu \mathrm{L}$, $22 \mu \mathrm{mol}$ ) was added. ${ }^{2} \mathrm{H}$ NMR spectra were recorded. Subsequently, the reaction mixture was treated with $(\mathrm{EtO}){ }_{2} \mathrm{MeSiD}(4.0 \mu \mathrm{L}, 25 \mu \mathrm{mol})$ and ${ }^{2} \mathrm{H}$ NMR spectra were recorded. Lateron, acetophenone $(4.0 \mu \mathrm{L}, 34 \mu \mathrm{mol})$ were added and ${ }^{2} \mathrm{H}$ NMR spectra 
were recorded. The reaction mixture was then quenched with glacial acetic acid $(100 \mu \mathrm{L})$ and filtered through silica. After evaporation of the solvent, the residue was extracted with diethyl ether and again filtered through silica. The filtrate was concentrated to a total volume of $0.5 \mathrm{~mL}$ and ${ }^{2} \mathrm{H}$ NMR spectra were recorded.

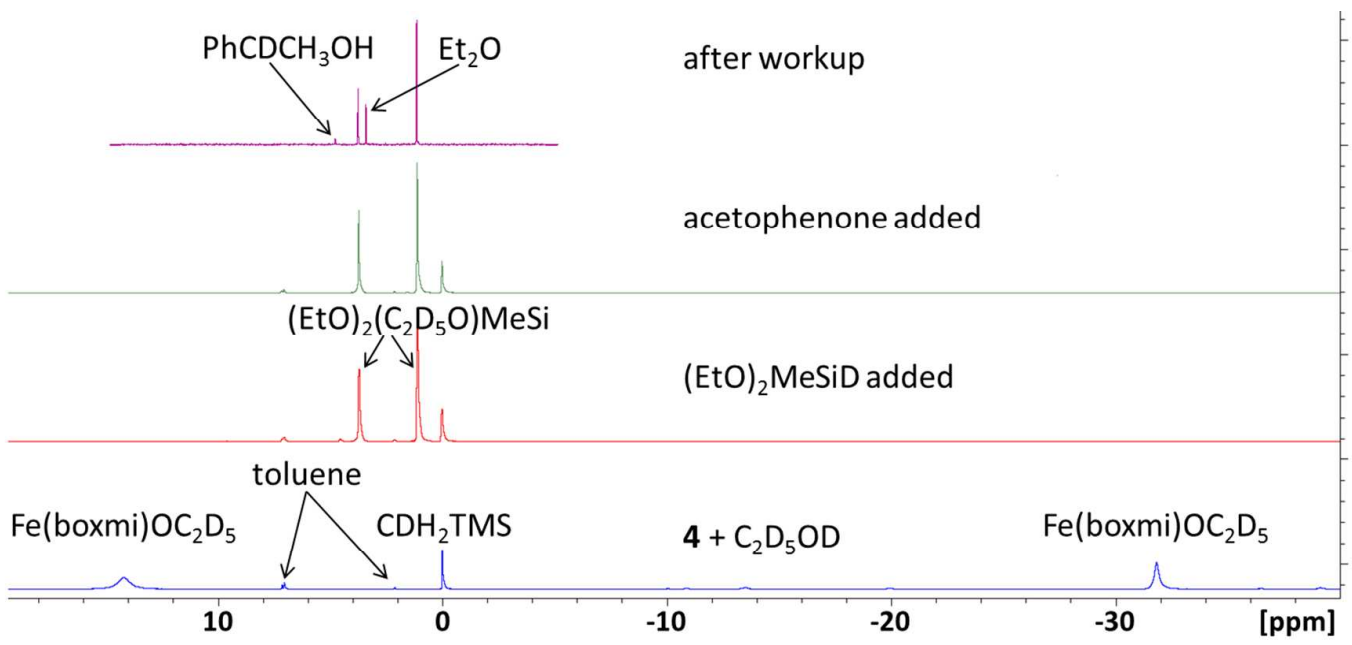

Figure S 27: ${ }^{2} \mathrm{H}$ NMR spectra of the reaction sequence to provide evidence for an iron hydride species.

Instead of adding acetophenone and subsequent workup of the reaction, we also probed the existence of an iron hydride species by adding ethylene (as a saturated solution in toluene). An exchange of the ethylene protons is expected in presence of an iron hydride species through an insertion into the Fe-H-bond and following $\beta$ hydride elimination. Therefore, an incorporation of deuterium atoms in ethylene is expected.

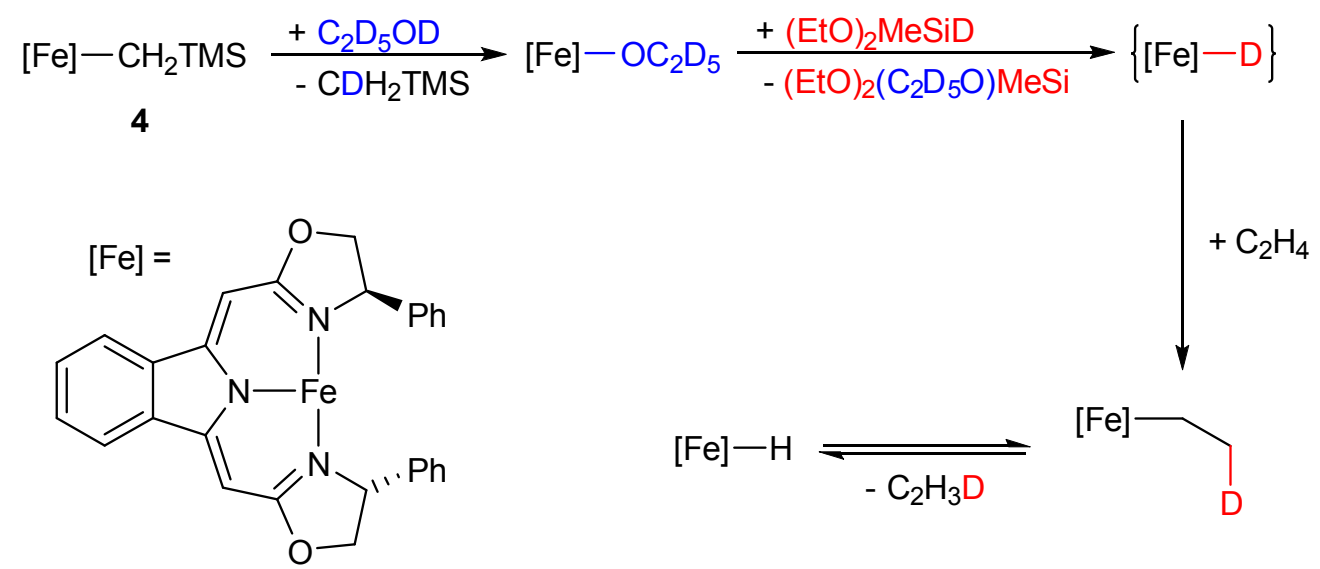




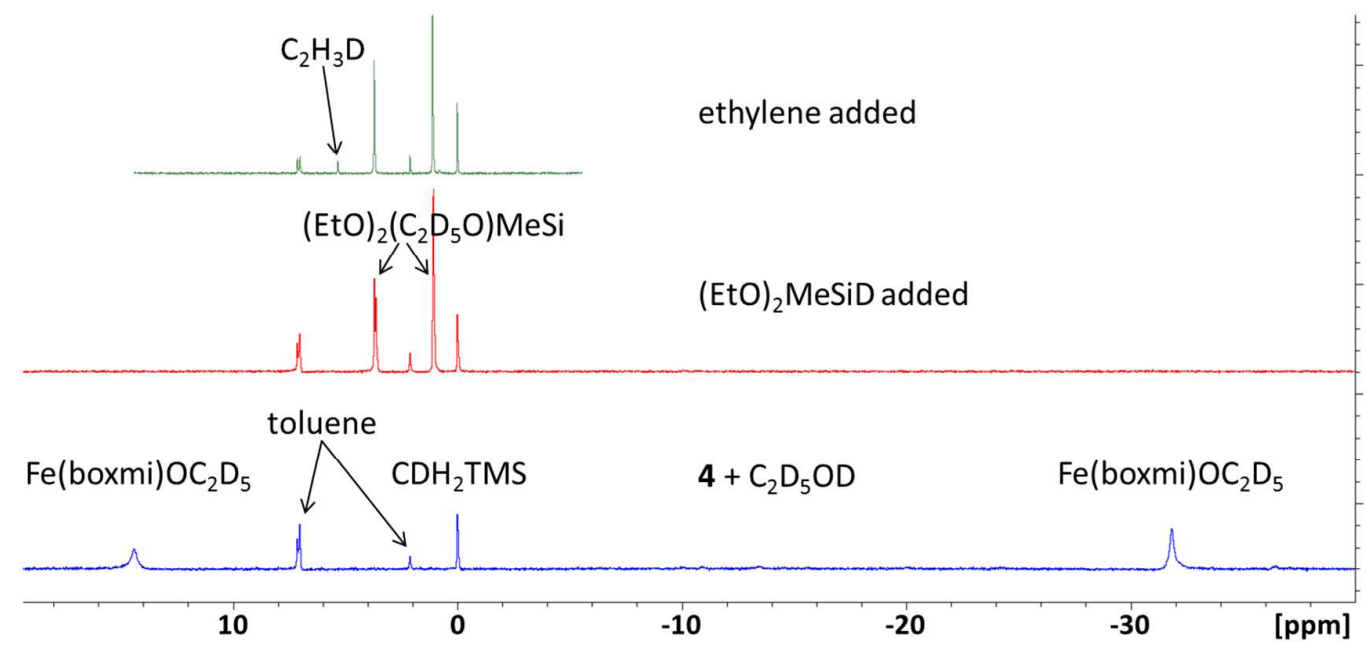

Figure S 28: ${ }^{2} \mathrm{H}$ NMR spectra of the reaction sequence to provide evidence for an iron hydride species by adding ethylene instead of acetophenone. 


\section{Computational Details}

All DFT calculations in this work were performed using the ORCA 3.0.3 program package. ${ }^{14}$ All structures were optimized in the gas phase using the BP86 functional ${ }^{15,16}$ alongside with a def2-TZVP basis $\operatorname{set}^{17}$ for all atoms. The RI approximation ${ }^{18}$ with the corresponding auxiliary basis $\operatorname{set}^{19}(\operatorname{def} 2-\mathrm{TZVP} / \mathrm{J})$ was applied for all optimization runs. The optimized geometries were subjected to frequency calculations on the same level of theory. All stationary points showed no imaginary frequency and all transition states showed one imaginary frequency. Gibbs free enthalpy corrections were calculated using the harmonic approximation at $233 \mathrm{~K}$ (BP86/def2TZVP level of theory) having all atoms (vide supra) in the same calculation to avoid any erroneous entropy effects, ${ }^{20}$ independent molecules were separated by at least $20 \AA$. The connection of two stationary points by a transition state was confirmed by an optimization run in each direction of the vibration associated with the imaginary frequency. Based on the optimized geometry, single point energy calculations were performed using a def2-QZVPP basis set ${ }^{17}$ for all atoms using the hybrid functionals B3LYP, ${ }^{21-23}$ PBE0,${ }^{24}$ and TPSSh. ${ }^{25,26}$ All calculations were run in gas phase as well as in a COSMO solvation model ${ }^{27,28}$ (toluene, $\varepsilon=2.4$, refractive index $=1.497$ ). The RIJCOSX ${ }^{29}$ approximation was used as implemented in ORCA 3.0.3. Since it was frequently shown, that the functional and basis set applied in this study give reliable results for geometries and energies, any additional screening of the DFT-method was beyond the scope of this study. ${ }^{30-32}$ Additionally, all four functionals showed the same results on semi-quantitative perspective, which is another indicator for the reliability of the results. Visualizations were made using ChemCraft. ${ }^{33}$

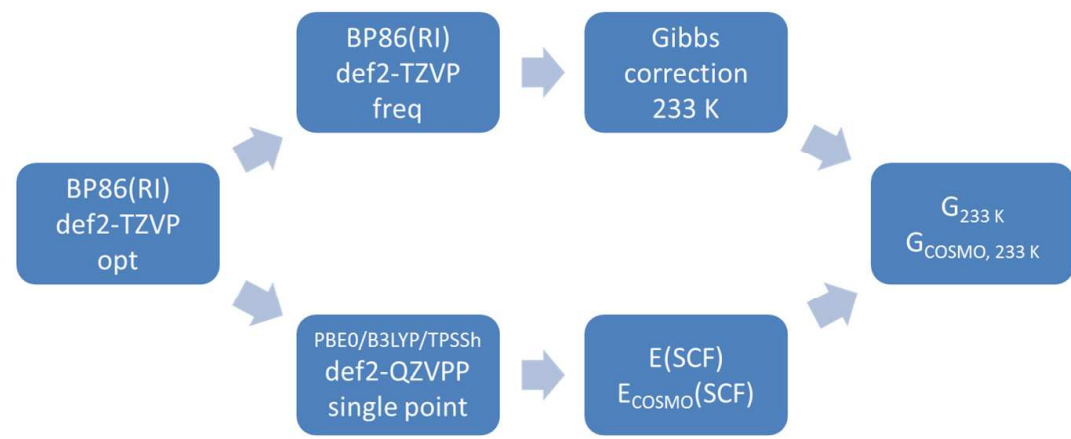

Figure S 29: Flow chart for the calculation of the Gibbs free enthalpies shown in the main paper. 


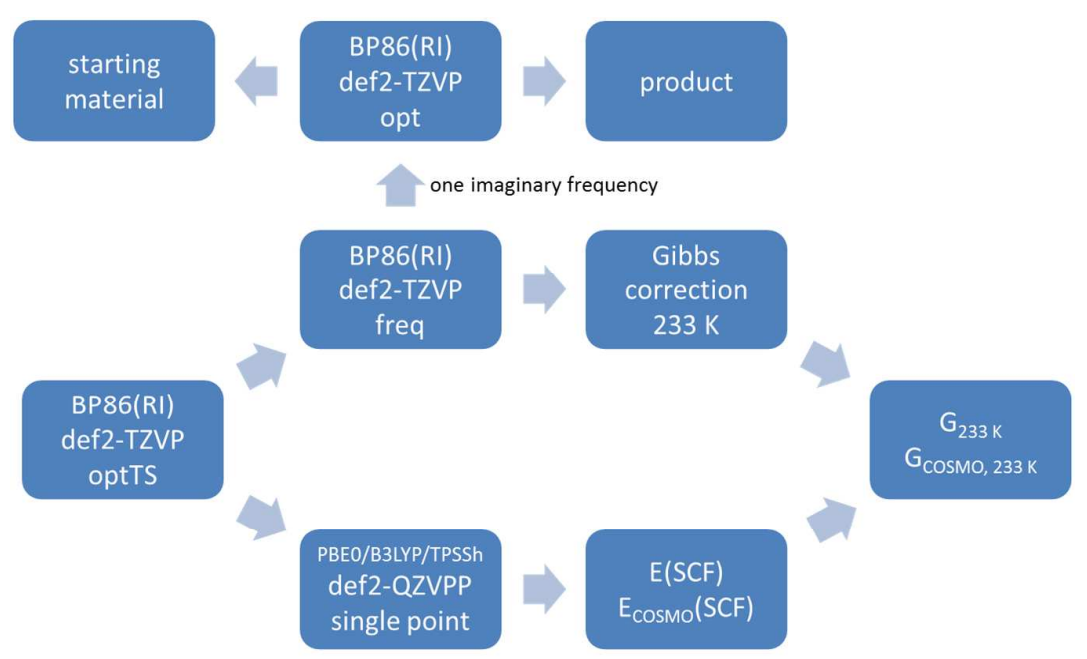

Figure S 30: Flow chart for the calculation of the Gibbs free enthalpies for the transition states.

All calculations were performed on the full complexes, no simplifications were used. The only exception is the substitution of $(\mathrm{EtO})_{2} \mathrm{MeSiH}$ with $(\mathrm{MeO})_{2} \mathrm{MeSiH}$, which saves significant amounts of computational cost because there are less conformational degrees of freedom available for the methoxy substituted silane than for the ethoxy substituted silane.

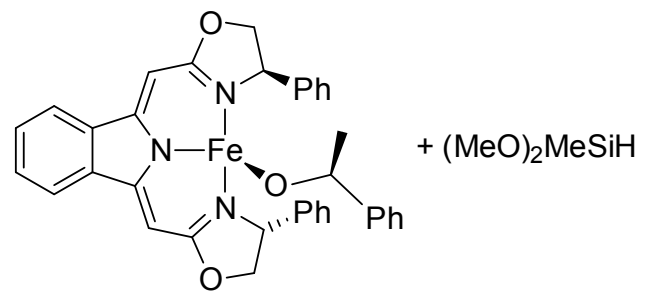

Scheme S 1: Overview of the molecules involved in the calculations, total 90 atoms $\left(\mathrm{C}_{39} \mathrm{H}_{41} \mathrm{FeN}_{3} \mathrm{O}_{5} \mathrm{Si}\right)$. 


\begin{tabular}{|c|c|c|c|c|c|c|c|c|}
\hline & & \multicolumn{3}{|c|}{ COSMO(toluene) } & \multicolumn{3}{|c|}{ Gas phase } & \multirow[b]{2}{*}{$\delta G_{233 K}$} \\
\hline & $\mathbf{E}_{\text {BP86/TZVP }}$ & $\mathbf{E}_{\text {B3LYP }}$ & $\mathbf{E}_{\text {PBE0 }}$ & $\mathbf{E}_{\text {TPSSh }}$ & $\mathbf{E}_{\text {B3LYP }}$ & $\mathbf{E}_{\text {PBE0 }}$ & $\mathbf{E}_{\text {TPSSh }}$ & \\
\hline Reactants & -3605.5652 & -3604.1928 & -3602.7575 & -3605.6603 & -3604.1735 & -3602.7371 & -3605.6414 & 0.6435 \\
\hline TS1 & -3605.5581 & -3604.1849 & -3602.7525 & -3605.6529 & -3604.1687 & -3602.7352 & -3605.6370 & 0.6428 \\
\hline Int1 & -3605.5730 & -3604.1828 & -3602.7527 & -3605.6643 & -3604.1775 & -3602.7341 & -3605.6495 & 0.6419 \\
\hline TS2_S & -3605.5627 & -3604.1762 & -3602.7530 & -3605.6527 & -3604.1625 & -3602.7383 & -3605.6393 & 0.6426 \\
\hline TS2_R & -3605.5576 & -3604.1717 & -3602.7485 & -3605.6486 & -3604.1576 & -3602.7334 & -3605.6346 & 0.6430 \\
\hline Int2_S & -3605.6168 & -3604.2397 & -3602.8122 & -3605.7095 & -3604.2268 & -3602.7982 & -3605.6968 & 0.6493 \\
\hline Int2_R & -3605.6185 & -3604.2410 & -3602.8146 & -3605.7109 & -3604.2287 & -3602.8013 & -3605.6988 & 0.6496 \\
\hline TS3_S & -3605.5937 & -3604.2121 & -3602.7986 & -3605.6899 & -3604.2028 & -3602.7883 & -3605.6806 & 0.6489 \\
\hline TS3_R & -3605.5988 & -3604.2201 & -3602.8028 & -3605.6948 & -3604.2102 & -3602.7919 & -3605.6850 & 0.6481 \\
\hline Int3_S & -3605.6036 & -3604.2238 & -3602.8118 & -3605.7029 & -3604.2141 & -3602.8013 & -3605.6933 & 0.6514 \\
\hline Int3_R & -3605.6030 & -3604.2248 & -3602.8124 & -3605.7035 & -3604.2146 & -3602.8013 & -3605.6934 & 0.6496 \\
\hline TS4_S & -3605.5988 & -3604.2192 & -3602.8044 & -3605.6962 & -3604.2088 & -3602.7932 & -3605.6860 & 0.6527 \\
\hline TS4_R & -3605.5934 & -3604.2084 & -3602.7938 & -3605.6858 & -3604.1989 & -3602.7833 & -3605.6763 & 0.6493 \\
\hline Products & -3605.6180 & -3604.2447 & -3602.8178 & -3605.7135 & -3604.2286 & -3602.8006 & -3605.6977 & 0.6439 \\
\hline
\end{tabular}

Table S 3: Energies of the calculated structures, all values are given in [a.u.]. 


\begin{tabular}{|c|c|c|c|c|c|c|c|}
\hline & & \multicolumn{3}{|c|}{ COSMO(toluene) } & \multicolumn{3}{|c|}{ Gas phase } \\
\hline & $\Delta \mathrm{G}_{\mathrm{BP} 86 / \mathrm{TZVP}}$ & $\Delta G_{B 3 L Y P}$ & $\Delta \mathrm{G}_{\mathrm{PBE} 0}$ & $\Delta \mathrm{G}_{\text {TPSSh }}$ & $\Delta G_{B 3 L Y P}$ & $\Delta \mathrm{G}_{\mathrm{PBE} 0}$ & $\Delta \mathbf{G}_{\text {TPSSh }}$ \\
\hline$[\mathrm{Fe}]-\mathrm{H}$ & 0 & 0 & 0 & 0 & 0 & 0 & 0 \\
\hline TS1 & 17 & 19 & 11 & 18 & 11 & 3 & 10 \\
\hline Int1 & -25 & 22 & 8 & -15 & -15 & 4 & -25 \\
\hline TS2_S & 4 & 41 & 9 & 17 & 26 & -6 & 3 \\
\hline TS2_R & 19 & 54 & 22 & 29 & 40 & 8 & 16 \\
\hline Int2_S & -120 & -108 & -128 & -114 & -125 & -145 & -130 \\
\hline Int2_R & -124 & -110 & -134 & -117 & -129 & -152 & -135 \\
\hline TS3_S & -60 & -36 & -94 & -64 & -63 & -120 & -89 \\
\hline TS3_R & -76 & -60 & -107 & -79 & -84 & -132 & -102 \\
\hline Int3_S & -80 & -61 & -122 & -91 & -86 & -148 & -115 \\
\hline Int3_R & -83 & -68 & -128 & -97 & -92 & -152 & -120 \\
\hline TS4_S & -64 & -45 & -99 & -70 & -69 & -123 & -93 \\
\hline TS4_R & -59 & -26 & -80 & -52 & -51 & -106 & -77 \\
\hline Products & -137 & -135 & -157 & -139 & -144 & -166 & -147 \\
\hline
\end{tabular}

Table S 4: Relative energies of the calculated structures, all values are given in $[\mathrm{kJ} / \mathrm{mol}]$. 


\section{Coordinates of all stationary points}

Acetophenone
\begin{tabular}{llll} 
E $=-385.037533720547$ & a.u. & \\
C & -2.44535497181578 & -0.21904219350538 & -0.09059067234496 \\
C & -1.04962314159572 & -0.23465021754910 & -0.05396338085807 \\
C & -0.32490325643205 & 0.96729728790011 & 0.01707464178864 \\
C & -1.02668943263233 & 2.18531305358979 & 0.05058196343368 \\
C & -2.41843396558474 & 2.20103440905646 & 0.01375846003384 \\
C & -3.13136269742766 & 0.99793193565669 & -0.05689781944863 \\
H & -2.99884345911849 & -1.15770272748078 & -0.14560088301280 \\
H & -0.52581028235025 & -1.19074315631411 & -0.08071370132327 \\
H & -0.44849280103628 & 3.10854261964643 & 0.10587218402206 \\
H & -2.95378390137034 & 3.15151046307033 & 0.04004578633416 \\
H & -4.22227595858072 & 1.00990560987649 & -0.08571713827993 \\
\hline C & 1.17591948704728 & 1.00664721436294 & 0.05941890264971 \\
\hline C & 1.94217197473303 & -0.30384757350511 & 0.01872968810824 \\
H & 3.01506283382053 & -0.08654367682789 & 0.05021367072646 \\
H & 1.67433198747748 & -0.94365089213672 & 0.87342366047029 \\
H & 1.70998701258193 & -0.86971089418311 & -0.89619290866568 \\
O & 1.77250057228411 & 2.07560873834297 & 0.12605754636624
\end{tabular}

\section{$(\mathrm{MeO})_{2} \mathrm{MeSiH}$}

$\mathrm{E}=-560.508732314638$ a.u.

$\begin{array}{cccc}\text { Si } & 0.62279471583718 & 1.41658171929594 & -0.65579930107388 \\ \text { H } & 1.26030670634581 & 1.95406833683709 & 0.59534984345779 \\ \text { O } & -0.94403042124495 & 1.92269690988557 & -0.77799465247348 \\ \text { O } & 0.62305275520530 & -0.23868456771695 & -0.51796073027476 \\ \text { C } & -1.92965670440283 & 1.66498820538055 & 0.22293553410395 \\ \text { H } & -1.59738389459136 & 2.01208901527236 & 1.21664716884756 \\ \text { H } & -2.84133444190408 & 2.21262129240520 & -0.05260426277507 \\ \text { C } & 1.63392289806741 & -0.99475798470115 & 0.14095239990204 \\ \text { H } & 2.04071184812353 & -0.46097334772527 & 1.01782463710625 \\ \text { H } & 2.46499982201436 & -1.22715045229730 & -0.54577649656122 \\ \text { C } & 1.48633078699581 & 2.02108996663280 & -2.19288077943793 \\ \text { H } & 1.00705940573072 & 1.61105574263321 & -3.09345044211901 \\ \text { H } & 1.45219622270930 & 3.11846014184547 & -2.25432583645276 \\ \text { H } & 2.54241186662122 & 1.71310442371140 & -2.19593475214379 \\ \text { H } & -2.16299251563566 & 0.59037608753143 & 0.28203777213412 \\ \text { H } & 1.19130424312825 & -1.94130878799035 & 0.48259306276019\end{array}$

\section{$(\mathrm{MeO})_{2} \mathrm{MeSi}\left(\mathrm{OCHCH}_{3} \mathrm{Ph}\right)$}

$\mathrm{E}=-945.598982539775$ a.u.
S 31

\begin{tabular}{|c|c|c|c|}
\hline & -1.07414890017580 & 2.29919734985977 & -0.05719483346144 \\
\hline C & -1.44238654368617 & 0.96148516591224 & -0.26346272351765 \\
\hline C & -2.59319776233714 & 0.47395395103893 & 0.36962838211462 \\
\hline $\mathrm{C}$ & -3.36811792644954 & 1.30405690003878 & 1.18524754575492 \\
\hline $\mathrm{C}$ & -2.99491671876238 & 2.635665888557171 & 1.38211377246758 \\
\hline $\mathrm{C}$ & -1.84337955699899 & 3.12954860930823 & 0.76017690534292 \\
\hline $\mathrm{H}$ & -0.16919335736415 & 2.68185129478302 & -0.53219303767823 \\
\hline $\mathrm{H}$ & -2.88299120482922 & -0.57019146720256 & 0.22573289545344 \\
\hline $\mathrm{H}$ & -4.25988738861573 & 0.90704102809262 & 1.67363089452821 \\
\hline $\mathrm{H}$ & -3.59452192610320 & 3.28491297175024 & 2.02224780104264 \\
\hline $\mathrm{H}$ & -1.54306652617140 & 4.16768919776974 & 0.91421011441600 \\
\hline $\mathrm{C}$ & -0.63751827879621 & 0.06165624393860 & -1.18834439618125 \\
\hline $\mathrm{H}$ & -0.88871933470607 & -0.98762611337835 & -0.95670351482863 \\
\hline $\mathrm{C}$ & -0.95147832524389 & 0.33361058531022 & -2.66060607832771 \\
\hline $\mathrm{H}$ & -0.38308538407529 & -0.35416624614678 & -3.30190706498472 \\
\hline $\mathrm{H}$ & -2.02420599433790 & 0.19647804081744 & -2.85714345955695 \\
\hline $\mathrm{H}$ & -0.68049339766197 & 1.36631076150648 & -2.92251765927490 \\
\hline $\mathrm{O}$ & 0.77808867491315 & 0.25434521499183 & -1.00206842171406 \\
\hline $\mathrm{Si}$ & 1.75376113691495 & -0.56581661240082 & 0.05056657024203 \\
\hline $\mathrm{O}$ & 3.29757205543605 & -0.45101027660002 & -0.51991203063959 \\
\hline $\mathrm{O}$ & 1.17827341819983 & -2.12397634732519 & 0.02397159900103 \\
\hline $\mathrm{C}$ & 1.80120547247203 & 0.13948139816704 & 1.77275460891870 \\
\hline $\mathrm{H}$ & 2.14017153536294 & 1.18440732886156 & 1.74690830280397 \\
\hline $\mathrm{H}$ & 0.80384578164120 & 0.11750961809673 & 2.23418440773046 \\
\hline $\mathrm{H}$ & 2.49393695670718 & -0.42046254860870 & 2.41763078413664 \\
\hline $\mathrm{C}$ & 3.68503923194789 & -0.76017398521635 & -1.86040326743983 \\
\hline $\mathrm{H}$ & 3.19203375235348 & -0.08787053659309 & -2.57977204665599 \\
\hline $\mathrm{H}$ & 3.43741225446572 & -1.80190662914136 & -2.12299102889579 \\
\hline $\mathrm{C}$ & 1.66680377108920 & -3.17901248921206 & 0.84977434674707 \\
\hline $\mathrm{H}$ & 2.74770077205228 & -3.34167138886498 & 0.70429610997321 \\
\hline $\mathrm{H}$ & 1.48219028997783 & -2.97652040107149 & 1.91826049202764 \\
\hline $\mathrm{H}$ & 4.77315183350006 & -0.62678576929814 & -1.93451624995628 \\
\hline $\mathrm{H}$ & 1.13414427728125 & -4.10007678275529 & 0.57444210841194 \\
\hline
\end{tabular}

\section{Reactants $[\mathrm{Fe}]-\mathrm{H}$}

$E=-2660.01898147124$ a.u.

Fe $0.42200801155956 \quad-0.14312804154103 \quad-0.04304159852681$

$\begin{array}{llll}\text { O } & 2.13349600518998 & 1.17782576486500 & -3.58648425646315\end{array}$

$\begin{array}{llll}\text { O } & -2.88809334301927 & -0.48629654513931 & 2.42654332911119\end{array}$

$\begin{array}{llll}\text { N } & -0.18554107610256 & 1.77015629923539 & -0.02553435941148\end{array}$

N $1.02233401714029 \quad 0.03474661855833 \quad-1.96839409943651$

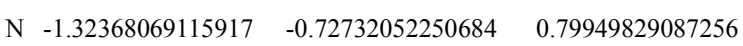


S 32
C $0.35337207394017 \quad 2.76497691585822 \quad-0.79881575154790$
C $\quad-2.70227779169754-3.90771525681471$
$-0.58943597530893$
$\begin{array}{llll}\text { C } & -4.17733472761621 & -2.11094818907074 & -2.12650640245537\end{array}$
H $\quad-2.11301644199440 \quad-4.61265867002996$
0.00261351951035
H $\quad-4.74739912747005 \quad-1.40197992296288 \quad-2.72953756506700$
C -3.46041263725332
$-4.37191763094230$
$-1.66711307165925$
C $\quad-0.14806201846335 \quad 4.05435324162553 \quad-0.31180118399194$
$\begin{array}{llll}\text { C } & -1.05119918526376 & 3.76115893987354 & 0.74225217955927\end{array}$
$\begin{array}{llll}\text { C } & -1.07715577728490 & 2.30009433673970 & 0.87144451149188\end{array}$
H -3.46435104847108
C -4.20321069263460
$-5.43601159378378$
$-1.90953345378096$
H -4.79397288773279
$-3.47356031989092$
C -3.42013236308439
$-3.83263179477171$
$-2.43788184002216$
C -1.71200304022256
1.42022956055216
H -2.41128006006467
4.78763252440513
2.22992837779610
C -1.45464806944614
4.57184643091546
1.04136619768310
H -1.95533325097440
6.10843292585854
1.56128860012660
C -0.55980884667605
6.92657310781343
$-0.00246992442244$
H $\quad-0.37836362165437$
6.39897981064756
$-0.27863557875152$
C 0.09784290821669
H $\quad 0.78791673068972$
5.61157856992227
$-0.69133995900136$
C 1.17393186213432
$2.51315258455073 \quad-1.88665555617314$
H 1.59468630539071
3.3451554642368
$-2.44780880274336$
C 1.42397530570601
1.2165952801152
$-2.41414232059303$
C 1.31457122330439
$-0.96614613135987$
$-3.02226321421180$
H $\quad 0.39128791261760$
C 2.36306079245431
$-1.12154478234045$
$-3.61071465039369$
H 3.39101315595100
$-0.22504288242300$
$-3.87898908777127$
H 2.24426424478774
$-0.4807309346946$
$-3.57548066426544$
C -1.88172373407150
$-0.37377191781140 \quad-4.95854171230904$
H -2.54837785274165
1.52168937705157
1.68699795273237
C -1.97310499881757
2.00039649744815
2.40142757834172
1.59935919691852
$\begin{array}{lll}\text { C } & -1.90106534583768 & -2.07693687853429\end{array}$
0.95771075531957
H $\quad-1.08754358729616 \quad-2.79495178541763$
1.14386991861123
C $\quad-2.77569807632748 \quad-1.91957603686778$
2.23203564756916

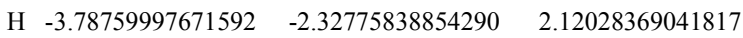

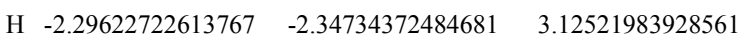
$\begin{array}{llll}\text { C } & 1.78509787813768 & -2.31419752711577 & -2.52174779414284\end{array}$
C $2.66392431856464 \quad-2.42410187185395 \quad-1.43320344513939$
H $2.96082350289242 \quad-1.52915087614092 \quad-0.88331580981751$
C $3.12563874023334 \quad-3.67788251755440 \quad-1.02425629557088$
$\begin{array}{llll}\text { H } & 3.79863294786507 & -3.74974668583493 & -0.16825646790724\end{array}$
$\begin{array}{llll}\text { C } & 2.72447205033900 & -4.83376500752688 & -1.70020214810338\end{array}$
H $3.08861982932579-5.81123175713655 \quad-1.37928815991782$
C $1.84704397518734 \quad-4.73263250894292 \quad-2.78321229264465$
H $\quad \begin{array}{lll}\text { H.52077945703081 } & -5.63026809226978 & -3.31118560272917\end{array}$
C $1.37721113931717 \quad-3.47986065628066 \quad-3.18543682415980$
H $\quad 0.67863558326518 \quad-3.40487746253745 \quad-4.02268631692653$
C $-2.67982443588535 \quad-2.54443255869339 \quad-0.26253550645133$
H $\quad-3.39065773970859 \quad-0.58222963612396 \quad-0.81946714656351$
$\begin{array}{llll}\text { H } & 1.67555970058423 & -0.39560637788431 & 0.99581736510137\end{array}$

\section{TS1}
$\mathrm{E}=-3045.0493183875$ a.u.
Fe -0.48904660592950
O 2.34078574713532
O $\quad-2.99279207980145$
0.06304750727605
$-0.54452807385086$
- -0.42553595932733
N -0.42553595932733
0.3416501572421
$-3.68260156474853$
N 1.15894775520314
1.44872621014946
2.60458361783149
N -1.89578155209081
41841578288
$-0.84532405177985$
C 0.29499556259946
0.36549266827543
$-1.78230463060145$
C $\quad-5.49126101852847$
$2.77125801730964 \quad-1.83272573447862$
H -6.02794894090335
$-1.34715292857155 \quad-0.69391434310393$
C -0.00442383216997
$-0.82774305163734 \quad-1.48982471368001$
C -0.89039363258867
$\begin{array}{ll}4.20312805278343 & -1.78395347074715\end{array}$
C -1.14143949505891
4.39111454690499
$-0.70082733031976$
C -1.35408864740623
$3.06642758949540 \quad-0.13068773542002$
H $\quad-2.04024758771655$
5.66848956514429
$-0.37102259905373$
C -0.91928652892431
$\begin{array}{ll}5.82411771736302 & 0.46347738799449\end{array}$
H -1.26990659166555
$6.75097846421082 \quad-1.13831104798743$
C -0.03417124922046
7.75671787426744
$-0.90126832460298$
H $\quad 0.28796403325564$
6.5638787918417
$-2.21556818317164$
C 0.43438750728787
7.42665573042134
$-2.80077191087511$
H 1.12231232181160
5.29044725692974
$-2.54541784025645$
C 1.18296890944952
5.15551369875716
$-3.38209199930299$
H 1.64267394378633
2.17375857790786
$-2.70880078093036$
C 1.53201351382753
$2.77743443432761 \quad-3.48885289413055$
C 1.8152165257423
0.80734116679710
$-2.67389324122813$
H 1.057327197230
$-1.36936794604366$
$-2.13574613584099$
C 2.304389006852
$-2.16800620291753$
$-2.12636917137226$
H 3.31026203364984
$-1.10278242836908$
$-3.58259801571084$
H $1.59313585565820 \quad-1.48257663781608 \quad-4.33316662424891$
$\begin{array}{llll}\text { C } & -1.94942495619859 & 2.81352601397661 & 0.96435211511748\end{array}$
H $\quad-2.39709945141034 \quad 3.65220902993809 \quad 1.49358856560082$ 


\section{S 33}
$\begin{array}{llll}\text { C } & -2.23753583841482 & 1.52345031154801 & 1.46172748269892\end{array}$
H $3.19741663617491 \quad-0.46754503322993$
6.09248293425585
$\begin{array}{llll}\text { C } & -2.56082650367212 & -0.70346613024169 & 1.69874076968415\end{array}$
H $3.84606295573253 \quad-2.72669255252788$
6.92175769733733
H $\quad-1.82197967921570 \quad-1.47200943198610 \quad 1.96792937005433$
C $-3.03431536695410 \quad 0.04784120753004 \quad 2.97280567221035$
Int1
H $\quad-4.06066313081953 \quad-0.19793913626727 \quad 3.27146072093662$
$\mathrm{E}=-3045.0642991815$ a.u.
H $\quad-2.34920175553990 \quad-0.10401245708669 \quad 3.82138789297886$
Fe -0.33454976826954
$\begin{array}{llll}\text { C } & 2.95388488977378 & -1.75717582799994 & -1.20177118463130\end{array}$
$\begin{array}{llll}\text { C } & 3.59371475432787 & -0.82753837665002 & -0.37410330814678\end{array}$
$\begin{array}{lllll}\text { O } & 2.16511380982967 & 0.27606749370671 & -3.58572150189390\end{array}$
$\begin{array}{lllll}\text { H } & 3.22419695279456 & 0.19778537003647 & -0.33946336336522\end{array}$
$\begin{array}{llll}\mathrm{O} & -3.36607844023113 & 0.93837305519851 & 2.67691544887242\end{array}$
$\begin{array}{llll}\text { C } 4.68328624523299 & -1.21075516681321 & 0.41429343976318\end{array}$
$\begin{array}{llll}\text { H } & 5.16941091464841 & -0.47432357068917 & 1.05652462687629\end{array}$
$\begin{array}{lllll}\mathrm{N} & -0.33954853084823 & 1.80276123053116 & -0.36264578271498\end{array}$
N $1.13862086759203 \quad-0.36497595832393 \quad-1.65753695321508$
C $5.14864483035388 \quad-2.52752818035425 \quad 0.38534469229238$
H $5.99949704875913 \quad-2.82384229979166 \quad 1.00071126824823$
C $4.51331723119496-3.46492307054128 \quad-0.43571592862818$
H $4.86631505313615 \quad-4.49753642267398 \quad-0.46443933227012$
C $3.42284204185685-3.08095243600060 \quad-1.21906239137723$
H $2.92772824461896 \quad-3.81972432080363 \quad-1.85548471077522$
$\begin{array}{llll}\text { C } & -3.70066670075452 & -1.37656400519393 & 0.94575293009508\end{array}$
$\begin{array}{llll}\mathrm{N} & -2.05161071646086 & -0.03915890979223 & 1.09954128186028\end{array}$
C $0.42815667260086 \quad 2.52427205743050 \quad-1.25962628302682$
C $\quad-5.43134711059517 \quad-1.20094995796273 \quad-1.29979544162269$
H $\quad-5.82148000752638 \quad-0.52436843794140 \quad-2.06229204004438$
C $0.23893801425335 \quad 3.95835726124950 \quad-1.01303728305985$
$\begin{array}{llll}\text { C } & -0.65241618746344 & 4.06356199182528 & 0.07307402520349\end{array}$
$\begin{array}{llll}\text { C } & -1.02141570528738 & 2.69311787562123 & 0.44554365032743\end{array}$
$\begin{array}{llll}\text { C } & -4.09592546929947 & -2.66901872267118 & 1.32040368508526\end{array}$
$\begin{array}{lllll}\mathrm{H} & -3.54367889580508 & -3.19605584208697 & 2.10342657898348\end{array}$
$\begin{array}{llll}\text { C } & -5.17994489881102 & -3.29417419650751 & 0.70050874407173\end{array}$
H $\quad-5.47033408077870 \quad-4.30280155549848 \quad 1.00040893960857$
$\begin{array}{lllll}\text { C } & -5.88350380800061 & -2.63199267136604 & -0.30966488892936\end{array}$
$\begin{array}{llll}\text { C } & -1.03127463833216 & 5.31443379706138 & 0.56803796455042\end{array}$
$\begin{array}{llll}\mathrm{H} & -1.72203712534324 & 5.40703905037479 & 1.40810665557589\end{array}$
C $-0.50066423389981 \quad 6.45585544837627 \quad-0.03805075741124$
$\begin{array}{llll}\mathrm{H} & -0.77953296683239 & 7.44342465371224 & 0.33288451419267\end{array}$
C $0.38859558210412 \quad 6.35063092430827 \quad-1.12148566954934$
H $-6.72764161558861 \quad-3.11905249786952 \quad-0.80059486931950$
H $\quad 0.78653080693315 \quad 7.25772607656708 \quad-1.57904368089931$
C $0.76755800103565 \quad 5.10110846212182 \quad-1.61861523437290$
$\begin{array}{llll}\text { C } & -4.40687597864153 & -0.72356560280888 & -0.07129351490745\end{array}$
H $1.45912162119275 \quad 5.02909139741464 \quad-2.46004612624718$
H $\quad-4.08942895621757 \quad 0.26976618636168 \quad-0.39014033710775$
$\begin{array}{lllll}\text { O } & 0.85816601396038 & -1.04386408238100 & 1.89673485730553\end{array}$
$\mathrm{H} \quad-1.18969815416496 \quad-1.24578626934783 \quad-1.30123408929086$
$\begin{array}{lllll}\text { C } & 1.24194904579865 & 2.00653872603941 & -2.24647395453737\end{array}$
H $1.71924939613150 \quad 2.69094879662073 \quad-2.94526042906789$
C $1.48479824518504 \quad 0.62517830347750 \quad-2.44650061161168$
$\begin{array}{lllll}\text { C } & 1.14345593215892 & -2.17802360625537 & 2.28383691684377\end{array}$
$\begin{array}{lllll}\text { C } & 0.74266705814555 & -3.39376586942241 & 1.47749278775947\end{array}$
C $1.64167213208957 \quad-1.62080672429991 \quad-2.24686455415775$
$\begin{array}{llll}\mathrm{H} & 0.20524693528171 & -4.12990013719855 & 2.09318862082792\end{array}$
$\mathrm{H} \quad 0.10903086362172 \quad-3.07779082073422 \quad 0.63988585808010$
$\begin{array}{llll}\mathrm{H} & 1.64007915472990 & -3.89116596768201 & 1.07806323296682\end{array}$
C $1.89434193337788 \quad-2.36559908082372 \quad 3.56591407475912$
$\begin{array}{llll}\text { C } & 2.26610264100534 & -3.63497177730195 & 4.04196035024537\end{array}$
$\begin{array}{llll}\text { C } 2.23942433757281 & -1.22758884964269 & 4.31753521828250\end{array}$
$\begin{array}{llll}\text { C } & 2.96595656103208 & -3.76341873500779 & 5.24282076738652\end{array}$
H $2.01383852565166-4.52965115387894 \quad 3.47216817457676$
$\begin{array}{llll}\text { C } & 2.93558958782722 & -1.35636978004939 & 5.51622289751869\end{array}$
$\begin{array}{llll}\text { H } & 1.94784050639652 & -0.24928948536324 & 3.93384049107467\end{array}$
$\begin{array}{llll}\text { C } & 3.30038783771785 & -2.62551984069609 & 5.98209070140044\end{array}$
H $3.25104336304442 \quad-4.75307206829727 \quad 5.60301950904676$
$\begin{array}{lllll}\mathrm{H} & 0.80070710595313 & -2.32646755074109 & -2.32642737081806\end{array}$
$\begin{array}{llll}\text { C } & 2.12202416785947 & -1.17196530074618 & -3.65599136654818\end{array}$
H $3.12583708934684-1.53306216891673 \quad-3.91204056561371$
H $\quad 1.41122505367080 \quad-1.45350524947360 \quad-4.44769794842037$
C $\quad \begin{array}{lll}-1.94188771644714 & 2.37869198908385 & 1.42854605630640\end{array}$
$\mathrm{H} \quad-2.38160889985213 \quad 3.18562001492978 \quad 2.01124802906615$
C $\quad-2.40814178768397 \quad 1.07058831465065 \quad 1.70240020117983$
C $\quad-2.91278263102186 \quad-1.12791625234822 \quad 1.59167431008135$
H $\quad-2.29153160536662 \quad-1.98868837315589 \quad 1.88137578415745$
C $\quad-3.56866417218788 \quad-0.48805069804972 \quad 2.84741355988080$
H $\quad-4.64682134839748 \quad-0.67403902536874 \quad 2.92212962586638$
H $\quad-3.06972006771383 \quad-0.79561353046548 \quad 3.77996384970317$
C $2.74300892622376 \quad-2.27782110392645 \quad-1.42910451382687$ 

C 3.62620482496640
H 3.48142440808021
C 4.66945850833005
$-1.52941553301003$
$-0.63989161216575$
$-0.45178977922092$
$-0.55112546133489$
H 5.34619428347935
$-2.15611866441295$
0.04763121495832
C 4.84497910272590
$-1.56029484981618$
0.66243360332498
H 5.66016651424184
C 3.96558484998625
H 4.08870243047084
C 2.92098798268411
H 2.2279447187710
C -3.92922077814279
C -4.40838129463754
H $\quad-3.99831319239198$
C -5.39294218551673
H $\quad-5.75015111850514$
C -5.90965840979466
H $\quad-6.67486451810705$
C -4.44759045392177
H -4.06162892464931
O 0.82344971550062
H -0.98803536330551
C $\quad 1.27446936050778$
C 0.97902273602277
H 0.57798906612593
H $\quad 0.25875141843183$
H 1.89970054782604
C 2.09020536556588
C 2.65727704977290
C 2.32242341802376
C 3.42759448755612
$-1.95952580587073$
H 2.50024678925955
C 3.09024995751490
$-3.35804965794564$
0.38701629216866
H 1.88530285810297
0.81962794493266
3.01916755427283
C 3.64691822035852
$-0.61428349763060$
5.56942512462138
H 3.86009224859779
H 3.25846980383293
$-2.74345080007269$
5.87945349844949
H 4.24878269419658
1.43791121186826
5.00337122220137
6.43903325935354

\section{TS2_S}

$\mathrm{E}=-3045.05396248708$ a.u.

Fe $-6.09198688231864 \quad-11.31198962467763 \quad-21.67978514672443$

$\begin{array}{llll}\text { O } & -8.38329401996387 & -13.40553222028279 & -24.64342768315096\end{array}$
O $\quad-6.75197122999299 \quad-8.13546791980434 \quad-18.95931211132116$

N $\quad-6.98251543311534 \quad-9.79751321592909 \quad-22.89106377810953$

N $\quad-7.10597835273698 \quad-12.67681581754036 \quad-22.91277859308878$

N $\quad-6.72107491101364 \quad-10.09079213497769 \quad-20.10770849191167$

C $\quad-7.34146827350292 \quad-9.95028115028271 \quad-24.20454723959241$

C $\quad-7.42624072260695 \quad-8.62360917948992 \quad-24.84217578649093$

$\begin{array}{llll}\text { C } & -7.14026019517318 & -7.68302973619026 & -23.82970975985469\end{array}$

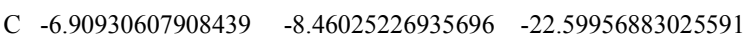

$\begin{array}{llll}\text { C } & -7.71985171896061 & -8.21126396461491 & -26.14091210763460\end{array}$

H $\quad-7.94245961501534 \quad-8.93254661664507 \quad-26.92929731927570$

C $-7.71903125601337 \quad-6.83842828052290 \quad-26.41681868537584$

H $\quad-7.94012953322895 \quad-6.49034912261418 \quad-27.42692604835165$

$\begin{array}{llll}\text { C } & -7.43814123059068 & -5.90298956795330 & -25.41015679726032\end{array}$

H $\quad-7.44214785732638 \quad-4.83876592105211 \quad-25.65096258063756$

$\begin{array}{llll}\text { C } & -7.15164864979830 & -6.31554311714869 & -24.10325774892056\end{array}$

H $\quad-6.93593363193925 \quad-5.57937461153039 \quad-23.32695134475394$

C $\quad-7.66279442288937-11.16018821952257 \quad-24.78386307452833$

H $\quad-8.05379932020070 \quad-11.18629615791865 \quad-25.79872899359572$

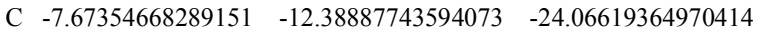

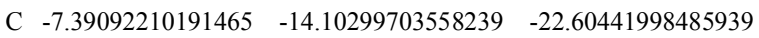

H $\quad-7.78343358412356 \quad-14.16979758204578 \quad-21.58052367844828$

C $\quad-8.48770251909045 \quad-14.45135195116224 \quad-23.64100874301227$

H $\quad-8.33594971178704 \quad-15.41675552461649 \quad-24.13748636877582$

H $\quad-9.49996439942566 \quad-14.40593222828773 \quad-23.21075131881028$

C $\quad-6.17418614266512 \quad-15.00560021699211 \quad-22.71218366556725$

C $\quad-5.84211117185252 \quad-15.86257700573712 \quad-21.65575707937249$

H $\quad-6.42839012924680 \quad-15.82764764637783 \quad-20.73462080876070$

$\begin{array}{llll}\text { C } & -4.76853418303041 & -16.75111738050417 & -21.76647037883030\end{array}$

H $\quad-4.52059808708340 \quad-17.40979210312624 \quad-20.93233892438554$

C $\quad-4.01249576682464 \quad-16.79121147640366 \quad-22.94004277397456$

H $\quad-3.17268327821372 \quad-17.48217724888043 \quad-23.02868958596563$

C $\quad-4.33355647073456 \quad-15.93636566586878 \quad-23.99962494116169$

H $\quad-3.74262084763144 \quad-15.95713446973815 \quad-24.91681494831630$

C $\quad-5.40509067644719-15.04908004083889-23.88564600629300$

H $\quad-5.64006793285146 \quad-14.37820657766372 \quad-24.71464561748957$

$\begin{array}{llll}\text { C } & -6.74567372341145 & -7.95320951647998 & -21.32413761329641\end{array}$

H $\quad-6.71522662433603 \quad-6.87736984680495 \quad-21.16638630111294$

$\begin{array}{llll}\text { C } & -6.72455609750680 & -8.77416045756433 & -20.16406218977615\end{array}$

C $\quad-6.85557922201856-10.50094549772221 \quad-18.69889562364247$

H $\quad-6.05319036321379 \quad-11.21229751585635 \quad-18.45512347827575$

C $\quad-6.63258270651916 \quad-9.16481445585175 \quad-17.93726317429185$

H $\quad-7.38888947241342 \quad-8.96471609940307 \quad-17.16854553310570$ 


\section{S 35}

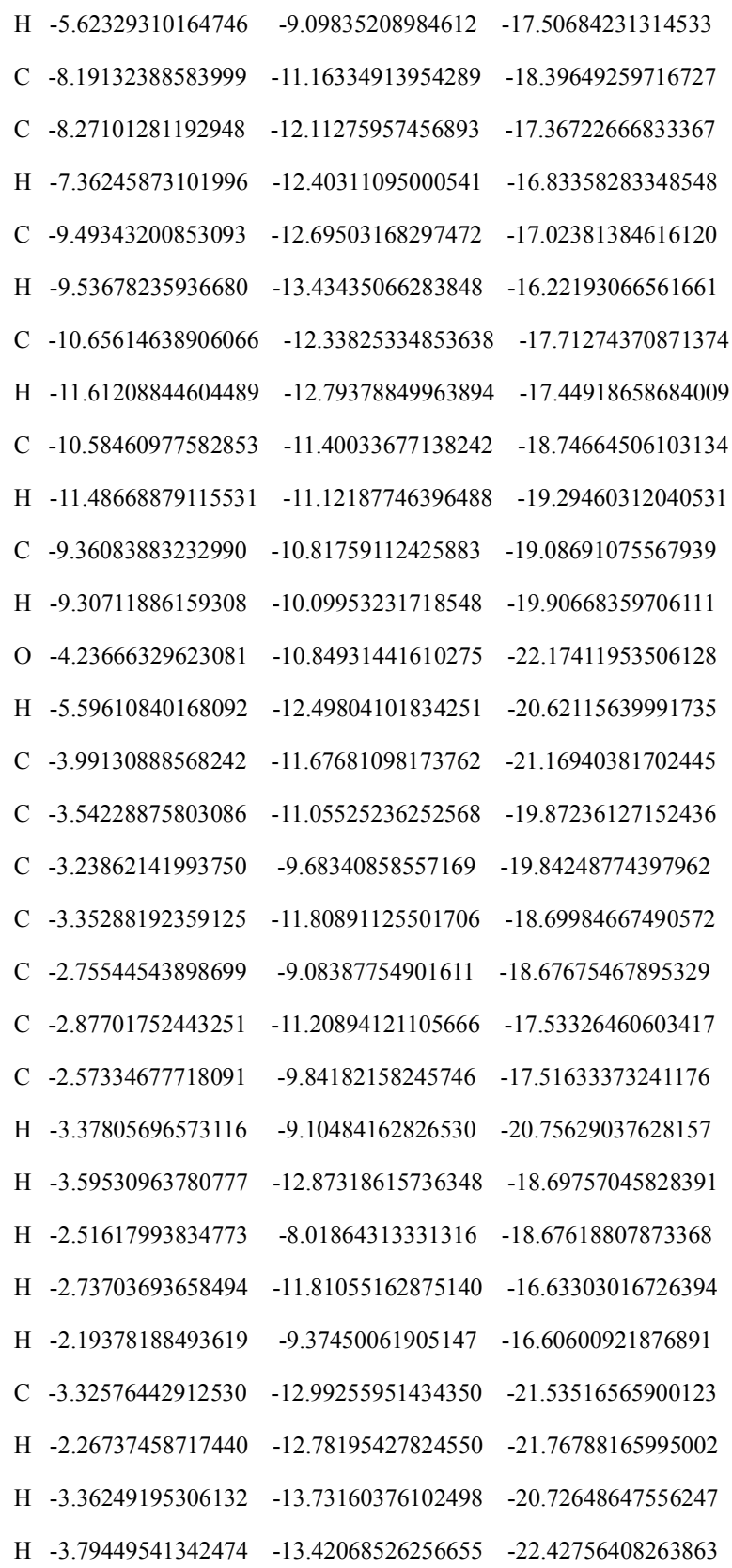

\section{TS2_S}

$\mathrm{E}=-3045.04882691365$ a.u.

Fe $-6.00199353993911 \quad-11.22595947690726 \quad-21.89991360838187$

$\begin{array}{lllll}\text { O } & -7.83404048505979 & -13.14977523194785 & -25.21992513918037\end{array}$

$\begin{array}{llll}\text { O } & -7.06595548924316 & -8.45906076758336 & -18.82881014766360\end{array}$

N $\quad-7.07839567557461 \quad-9.66898762291892 \quad-22.92434179252607$

N $\quad-6.96069174689747 \quad-12.51226233763115 \quad-23.22253345135134$

N $\quad-6.70611712075830 \quad-10.24386048086694 \quad-20.18300471481110$

C $\quad-7.23818299971328 \quad-9.69469859203445 \quad-24.28208245741466$

C $\quad-7.40729446320652 \quad-8.31562858840860 \quad-24.77881227759091$

$\begin{array}{llll}\text { C } & -7.36757969665645 & -7.48262862480258 & -23.64005767588959\end{array}$

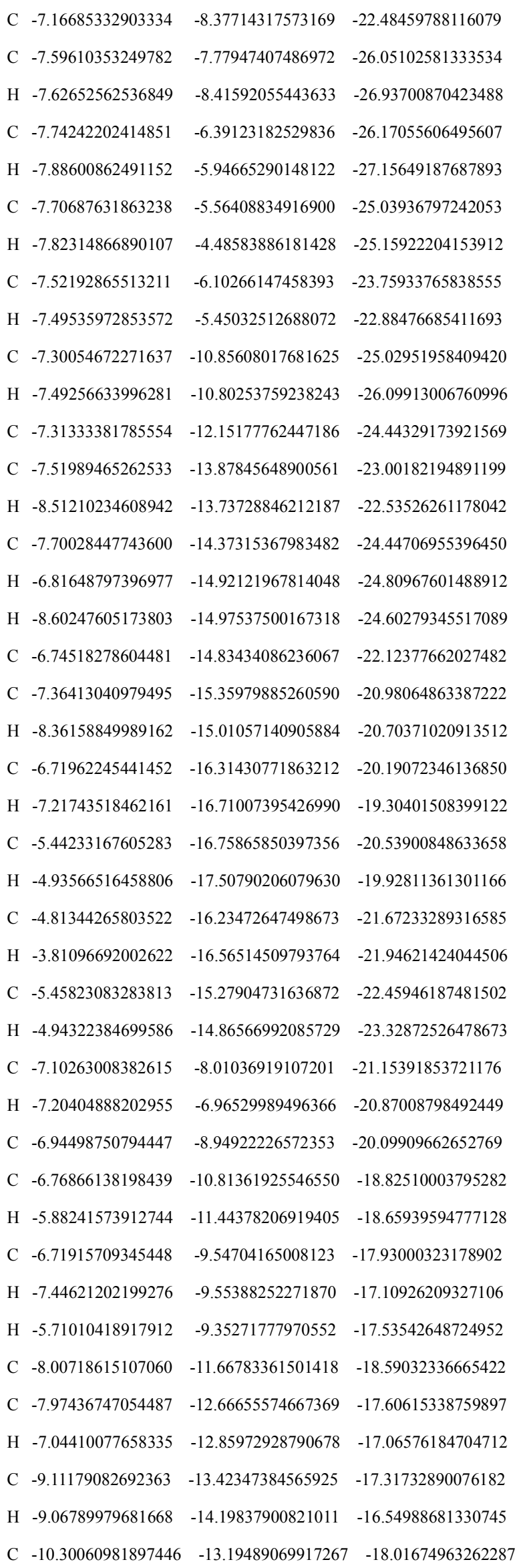


H $\quad-11.19061500034139-13.78599334860288 \quad-17.79474338582622$

C $-10.33895836408310 \quad-12.20949629700057 \quad-19.00702890047202$

$\begin{array}{llll}\mathrm{H} & -11.26109159749397 & -12.02891231745471 & -19.56248652357348\end{array}$

C $\quad-9.19961287876751 \quad-11.45158916212033 \quad-19.29300418599525$

H $\quad-9.23009090348988 \quad-10.69516214243242 \quad-20.07856845148126$

$\begin{array}{lllll}\text { O } & -4.30932471922975 & -10.39659100534135 & -22.49195026973659\end{array}$

H $\quad-5.21233187817947 \quad-12.32811813064767 \quad-20.94279136521785$

$\begin{array}{lllll}\text { C } & -3.82130232622715 & -11.36558095020841 & -21.72888309851949\end{array}$

$\begin{array}{lllll}\text { C } & -3.12389506979987 & -12.48862164677653 & -22.44760139225442\end{array}$

C $\quad-3.15020061663460 \quad-12.51578422868016 \quad-23.85399086560623$

C $\quad-2.37271944232713 \quad-13.46971313493240 \quad-21.77679551450881$

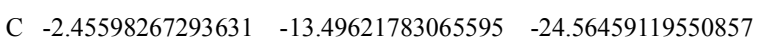

H $\quad-3.70933293694952 \quad-11.73802171375092 \quad-24.37467839798507$

C $-1.67027582128929-14.44387009536135 \quad-22.48768771156580$

H $\quad-2.33756582018536 \quad-13.47934262939295 \quad-20.68704212907090$

C $-1.71023873331668 \quad-14.46555527720795 \quad-23.88587485593553$

H $\quad-2.48470830196679 \quad-13.49519597612509 \quad-25.65613599213167$

H $\quad-1.08522872091961 \quad-15.19019254808260 \quad-21.94649043423004$

H $\quad-1.15792673147797 \quad-15.22581203139826 \quad-24.44089349844458$

C $\quad-3.21041773015666 \quad-10.91762958462190 \quad-20.40695277404506$

H $-3.83879165111965-10.13794727305963 \quad-19.96039335144760$

H $\quad-3.09247902530935 \quad-11.73783086195394 \quad-19.68820051035832$

H $\quad-2.21674377744022 \quad-10.48179903897044 \quad-20.60766381271775$

\section{Int2_S}

$\mathrm{E}=-3045.1080524421$ a.u.

Fe $-0.07889529552186 \quad-0.69914266692299 \quad 0.37088354577049$

$\begin{array}{lllll}\text { O } & 2.57751597314948 & 0.54810790143323 & -2.55635283532641\end{array}$

$\begin{array}{llll}\text { O } & -3.86268041062316 & -0.58514222960777 & 2.11111835993594\end{array}$

$\begin{array}{lllll}\mathrm{N} & -0.37335867301650 & 1.30262625455347 & 0.47282677386099\end{array}$

N $0.95973411596985 \quad-0.49251697028258 \quad-1.34886832836218$

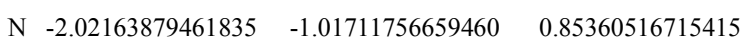

$\begin{array}{lllll}\text { C } & 0.47734580942224 & 2.23300450059563 & -0.06387088286049\end{array}$

$\begin{array}{llll}\text { C } & -4.36704418263886 & -1.63748758321267 & -2.72032015633473\end{array}$

H $\quad-4.67726345922604 \quad-0.79520609028059 \quad-3.34117337415707$

C $0.09322357602693 \quad 3.55890108593930 \quad 0.43441928086303$

C $-1.05350582417296 \quad 3.36384119478804 \quad 1.24534721203775$

C $-1.33643915903304 \quad 1.92332403127343 \quad 1.22182811451575$

C $\quad-1.67595210645045 \quad 4.44524282279183 \quad 1.87126823320831$

$\begin{array}{llll}\mathrm{H} & -2.56063953001269 & 4.30551703120258 & 2.49496722990131\end{array}$

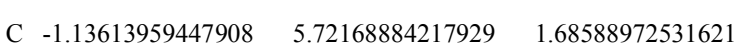

$\begin{array}{llll}\mathrm{H} & -1.60263549715742 & 6.58073546169130 & 2.17042759530966\end{array}$
C $0.00033695453929 \quad 5.91501713576706$

H $\quad 0.40112871851581 \quad 6.92171325271396$

C 0.62284784923844

4.83693593541510

H 1.50374385761626

C 1.47392796498408

4.99804085315113

H 2.13890658586940

C 1.63423101177598

C 1.31529657494183

H 0.54448259196718

C 2.64454986583935

H 3.51273699237744

H 2.76902651085047

C -2.40585147511561

H -3.12566552329103

C -2.69663476146609

C -2.84269954327463

H -2.22988135600415

C -3.93894382315943

H $\quad-4.95351821500154$

H -3.74015212415319

C 1.41784754723498

C 1.95305632385837

H 2.23306416202740

C 2.08903182182267

H 2.49718998677655

C 1.70359597667727

H 1.81474014403323

C 1.16534150265349

H 0.85093593909026

C 1.01902674110486

H 0.58304027038280

C -3.39745335813468

C -3.55374604719641

H $\quad-3.22240596416998$

C -4.11798649301351

H -4.22859612492620

C -4.52944041842983

H -4.96793917482030

C -3.80354301816050

H -3.66390097531439

O 1.0819909572803

C 1.22548057929580
1.91041448000884

0.88308655582308

0.75513720090527

0.24656164427174

$-0.37718934439703$

$-0.97035317797286$

$2.68972464347539-1.33752346476100$

$0.62833191292033-1.56424382206843$

$-1.44257162066415 \quad-2.43060140945094$

$-1.36341421226448 \quad-3.21998900452686$

$-0.84966739344752 \quad-2.94125975368179$

$-1.30912126225962 \quad-2.44149599508946$

$-0.89976917115575 \quad-4.02914726372457$

$1.24905259289850 \quad 1.79115393172554$

$1.79527929094146 \quad 2.39742005228662$

$-0.12340395529847 \quad 1.56315650788252$

$-2.24147538441626 \quad 0.74450158857055$

$-3.11380027497008 \quad 1.01721455734135$

$-2.00324258198522 \quad 1.81814956164542$

$-2.22336204099566 \quad 1.46445557873417$

$-2.55577592656026 \quad 2.74901239218438$

$-2.89455748947967-2.01971298134925$

$-3.27158783158497 \quad-0.77847147835838$

$-2.51018305462310 \quad-0.04749054352192$

$-4.62436127931105 \quad-0.45436302073592$

$-4.90407846798511 \quad 0.51822733893494$

$-5.61359194456087 \quad-1.36349173381638$

$-6.66852572775857 \quad-1.10689214166984$

$-5.24474605180230 \quad-2.59934849089118$

$-6.00932067737630 \quad-3.31175416660862$

$-3.89322279411895 \quad-2.92014733240118$

$-3.60755208300808 \quad-3.88085297219326$

$-2.46972219538963 \quad-0.65342858793109$

$-3.77817246892397 \quad-1.13193958142233$

$-4.61901972473643 \quad-0.51738919245349$

$-4.01752437748499 \quad-2.38746285586180$

$-5.04228899325775 \quad-2.74616097852528$

$-2.94622864418001-3.18459038183395$

$-3.12951829809812-4.16678560535123$

$-1.40029883771025-1.46439235582868$

$-0.37570042904929-1.11572554562230$

$-1.23064051888141 \quad 1.72791593278331$

$-2.68161890645053 \quad 3.64155727683999$ 
S 37
H 1.08271184298605
H $\quad 0.57430577541821$
H 2.27214435206922
C 1.76247477234468
C 1.41586612156815
C 2.92617562520213
C 2.21700143017334
H $\quad 0.50043977880750$
C 3.73126430571720
H 3.18117949498710
C 3.38211630572959
H 1.92765626147474
H 4.63443842094923
1.58223905715719
H 4.00825331684094
C 0.90164724235116
H -0.15600102935940
2.34189281724649
$-1.26748132118705$
$-1.04716932370111$

\section{Int2_R}

$\mathrm{E}=-3045.1097980323$ a.u.

Fe 0.48513246889169

O 2.08566650521789

O $\quad-2.66837287740872$

$-0.32057756596413$

$-0.12207292691576$

$.21023522953530 \quad-3.63645834744022$

N $\quad-0.10345102009921$

$-0.74010218502619$

2.55565388417066

N 1.04606625961908

N $\quad-1.19359948120829$

1.61160116985705

0.03847092504813

C 0.41083787960383

C -4.2200609495108

H $\quad-4.81720218584088$

C $\quad-0.06127192573596$

C -0.91813979569270

C -0.94580024030266

C -1.54193550153831 $-0.02780577193945$

$-0.92424376493853$

$-2.04058395878991$

0.84057405142727

2.64141286752940

$-0.70437958142316$

$-2.22096498729799$

$-1.94426734395425$

$-1.49247321106686$

$-2.49568269970697$

3.90911231511764

3.56908560106907

$-0.13498272064357$

0.94243285893144

2.10195440398047

1.00211790783429

Н -2.20641408429550

4.56363118412445

1.69805058540555

4.31140511572673

2.52632592435571

C $\quad-1.29416577268889$

5.90060003728187

1.37211218669187

H -1.76707373109140

6.69438330332143

1.95229072762478

C -0.44461013006998

6.23770564682874

0.30504471629310

H $\quad-0.26938084783173$

7.28891933951421

0.07114749084635

C $\quad 0.17587287857440$

H $\quad 0.83074955535831$

5.24619008220067

$-0.46085766209612$

C 1.19022560151883

5.51847625221495

$-1.29032055733056$

H 1.58966044361716

2.4451926188200

$-1.83264308158695$

C 1.42566059564573

$3.30558122667946 \quad-2.36603700589088$

$1.17920456029643 \quad-2.43545158714136$
C $\quad 1.30547516598640-0.96963739427088$

H $\quad 0.36030328307802-1.11176009494747$

C 2.30767218625138

H 3.35016000522096

H 2.13818047494368

C -1.70709644967685

H $\quad-2.33513951100557$

C -1.80079069662686

C -1.75654845099380

H $\quad-0.93139223490414$

C -2.53561664633355

H $\quad-3.54304393701561$

Н -1.97131688596241

C 1.82260851987925

C 2.75996507096068

Н 3.06538400163020

C 3.27505610189709

H 4.00005275778247

C 2.86654458144909

Н 3.27353461148376

C 1.92732983146836

H 1.59390096010323

C 1.40705854790777

H $\quad 0.66338256164054$

C -2.61660219956750

C -2.67731866698946

H $\quad-2.06311639178509$

C -3.50572870257612

H $\quad-3.53845794901597$

C -4.28229407881185

H $\quad-4.92786332763889$

C -3.39237283595601

Н $\quad-3.33641639375316$

O 1.98785031194255

C 2.10869892088355

H 1.49306405191186

C 3.57643204778929

H 4.21903958426289

H 3.89477117783612

H 3.7102715920947

C 1.6286251186014

C 1.7172647091559
$-3.15605644965950$

$-3.71194596751895$

$-0.17129720137283-4.01821776378625$

$-0.43620703016925-3.77946380826808$

$-0.26090132281929-5.09759491631548$

$1.29288317554179 \quad 1.82902969320090$

$1.74465180421522 \quad 2.59426237221657$

$-0.12221686454902 \quad 1.70138680636680$

$-2.28246285991749 \quad 0.99399390161585$

$-3.00157152812894 \quad 1.10668824285580$

$-2.16943437020182 \quad 2.33146000285180$

$-2.60078925285550 \quad 2.29250100255164$

$-2.59432753584016 \quad 3.17476960114481$

$-2.33173075493388 \quad-2.74827873021943$

$-2.47795209752631 \quad-1.71398725263214$

$-1.61023257535367 \quad-1.12577882857683$

$-3.74012507200471-1.40654196780795$

$-3.84190250091896-0.59720762388299$

$-4.86689417093537 \quad-2.12626317819594$

$-5.85003989424761-1.88392705093575$

$-4.72923182594453 \quad-3.15159550603765$

$-5.60425120961471 \quad-3.71203525049086$

$-3.46882764586183 \quad-3.45557675067239$

$-3.36534936441330-4.24985741740929$

$-2.71163185171312-0.18488114965457$

$-4.06743294545392 \quad-0.53849010310662$

$-4.79180272084241 \quad 0.00249505995340$

$-4.49931632796853 \quad-1.57699879788414$

$-5.55786481403802-1.84074267546026$

$-3.57587808885983-2.28232449460909$

$-3.90970066521534 \quad-3.09621677095332$

$-1.79143423522190 \quad-0.90382293632525$

$-0.73042858440897 \quad-0.65528020050007$

$-0.59656511875046 \quad 0.95152373683831$

$-0.41738582922627 \quad 2.34037097895471$

$0.44611495705793 \quad 2.67409708907133$

$-0.09967024297497 \quad 2.68174654209809$

$-0.93970532433010 \quad 2.37997831007867$

$0.79915441486228 \quad 2.13534973901167$

$0.07249526305254 \quad 3.76023034814896$

$-1.63407632469728 \quad 3.13233823171457$

$-2.92509653142777 \quad 2.59282858663800$ 


$\begin{array}{cccc}\text { C } & 1.11778155955534 & -1.48572305264434 & 4.43021941740561 \\ \text { C } & 1.31239740909168 & -4.03930758372895 & 3.33440508124219 \\ \text { H } & 2.09812555440471 & -3.04117864702076 & 1.57672882743916 \\ \text { C } & 0.71094350779139 & -2.59617261668464 & 5.17523443852800 \\ \text { H } & 1.03090311824887 & -0.48409965916059 & 4.86011689329000 \\ \text { C } & 0.80787125235479 & -3.88011062766525 & 4.62931210597674 \\ \text { H } & 1.39297102139934 & -5.03821142267909 & 2.90011249741844 \\ \text { H } & 0.31195740265916 & -2.45867217236832 & 6.18223314135068 \\ \text { H } & 0.49124271221442 & -4.74941212868598 & 5.20838915723068\end{array}$

\section{TS3_S}

$\mathrm{E}=-3605.59368790179$ a.u.

O 3.05385584939657

O -2.23773911300251

N 0.09330940941880

N $1.72204836621702-1.21374693941003 \quad-0.95896234601761$

$\begin{array}{llll}\mathrm{N} & -1.37198613184276 & -0.37195600855229 & 1.72137086233863\end{array}$

C $0.73825275771154 \quad 1.56301520509118 \quad-1.45463108480116$

$\begin{array}{llll}\text { C } & -5.46928251237311 & -1.33520573663622 & 0.75404781155290\end{array}$

$\begin{array}{llll}\text { H } & -6.02340526146610 & -0.72163484468824 & 0.04140374177464\end{array}$

$\begin{array}{llll}\text { C } & 0.42246820292487 & 2.98568721848543 & -1.62360713136414\end{array}$

$\begin{array}{llll}\text { C } & -0.36859543617552 & 3.35070830688828 & -0.51689931447392\end{array}$

C $-0.56508069832813 \quad 2.13029313224053 \quad 0.27188309203312$

C $\quad-0.81191103530919 \quad 4.66619825651341 \quad-0.35609723617023$

H $\quad-1.42413295706276 \quad 4.95871496615248 \quad 0.49876917025983$

$\begin{array}{llll}\text { C } & -0.45420261302693 & 5.60790790802483 & -1.32394815897581\end{array}$

H $\quad-0.79060824826764 \quad 6.64091227442346 \quad-1.22292633802013$

C $0.33308131228384 \quad 5.24296234786443 \quad-2.42996732970182$

H $\quad 0.59528782119367 \quad 5.99728754372863 \quad-3.17342163403890$

$\begin{array}{llll}\text { C } & 0.78276415191047 & 3.92984864987338 & -2.58862632216974\end{array}$

$\begin{array}{llll}\mathrm{H} & 1.39479529475284 & 3.65661780907503 & -3.44992030629857\end{array}$

$\begin{array}{llll}\text { C } & 1.61694019765059 & 0.86336848618247 & -2.25363527656622\end{array}$

$\begin{array}{llll}\mathrm{H} & 2.05105198579055 & 1.35470418484696 & -3.12182184859304\end{array}$

$\begin{array}{llll}\text { C } & 2.08377424046974 & -0.43842610109984 & -1.95950682302968\end{array}$

C $2.62845208621745-2.38416932440305 \quad-0.96751424593455$

H $2.02758001484796-3.29471013527872 \quad-0.84339001746953$

C $3.22995463912502-2.32508922818436 \quad-2.39090421619249$

H $4.30024351326835 \quad-2.55819540305511 \quad-2.43313823103546$

H $2.67239536094731-2.95656155442442 \quad-3.09862068440756$

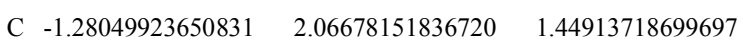

$\begin{array}{llll}\mathrm{H} & -1.66153590777203 & 2.98609881997026 & 1.88928367820547\end{array}$

$\begin{array}{llll}\text { C } & -1.58375131130878 & 0.86702635568273 & 2.12892399155203\end{array}$ $\begin{array}{llll}\text { C } & -2.12881703869768 & -1.25117762691239 & 2.64614467228979\end{array}$

H $\quad-1.52934195778895 \quad-2.13224512341508 \quad 2.90447509204446$

$\begin{array}{llll}\text { C } & -2.30454110982378 & -0.34203582350143 & 3.88097036723413\end{array}$

H $\quad-3.26959411766978 \quad-0.45553919096867 \quad 4.38771002031095$

H $\quad-1.47851015917438 \quad-0.46137834253194 \quad 4.60021141315622$

$\begin{array}{llll}\text { C } & 3.70235501073777 & -2.31987807374153 & 0.11188219138951\end{array}$

C $4.13566381464964 \quad-1.10569240979282 \quad 0.66103261461821$

H $3.65079687967633 \quad-0.17652811981096 \quad 0.35797346871580$

$\begin{array}{llll}\text { C } & 5.17120499191191 & -1.07928095116717 & 1.60044247181180\end{array}$

H $5.49689625123049 \quad-0.124668662618364 \quad 2.01792236092556$

C $5.78899334653701 \quad-2.26578822952135 \quad 2.00305143779001$

$\begin{array}{llll}\text { H } & 6.59562616182403 & -2.24425167010908 & 2.73762629183444\end{array}$

$\begin{array}{llll}\text { C } 5.36266125083527 & -3.48240038587557 & 1.46196021846084\end{array}$

H $5.83199372779046 \quad-4.41622987843721 \quad 1.77624157174593$

C $4.32751838310511 \quad-3.50645851511943 \quad 0.52564294239118$

H $3.99595139708809 \quad-4.46215535090221 \quad 0.11163969180112$

$\begin{array}{llll}\text { C } & -3.46938314613644 & -1.70575566549721 & 2.08457814650338\end{array}$

$\begin{array}{llll}\text { C } & -4.03889012863969 & -2.89482441900823 & 2.56475778017389\end{array}$

H $\quad-3.47882233888770 \quad-3.50925185218024 \quad 3.27426541299783$

C $\quad-5.30884727161602 \quad-3.30024890721641 \quad 2.14863073925335$

H $\quad-5.73340081995096 \quad-4.23139687103654 \quad 2.52805076947031$

$\begin{array}{llll}\text { C } & -6.03043475684190 & -2.51842865692915 & 1.24207127365999\end{array}$

H $\quad-7.02191746036204 \quad-2.83320867320559 \quad 0.91281388699487$

$\begin{array}{llll}\text { C } & -4.19684478680002 & -0.93244611025954 & 1.17029439043976\end{array}$

H $\quad-3.76004856998781 \quad-0.01536696493133 \quad 0.77280639621679$

$\begin{array}{llll}\text { O } & -0.33141712721997 & -2.93760406408596 & 0.30196670281436\end{array}$

$\begin{array}{llll}\text { C } & 0.42457507843449 & -5.24317507248648 & 0.62026213618357\end{array}$

H $0.32683230828597 \quad-6.10775415214709 \quad 1.29316961006062$

H $0.00913192568929-5.49650635276737 \quad-0.36252197301149$

H $1.49563137767129 \quad-5.02559012268484 \quad 0.50166452392352$

$\begin{array}{llll}\text { C } & 0.29194851595196 & -3.67884869953452 & 2.57283768962113\end{array}$

C $-0.11946617160269-4.40180093899186 \quad 3.70525046815196$

C $1.27199227403662 \quad-2.69346565250570 \quad 2.73807997899615$

C $0.42586252572045 \quad-4.14293015818988 \quad 4.96429427360634$

H $\quad-0.88531677674991 \quad-5.17478673263515 \quad 3.59655448586058$

C $1.81950437849482 \quad-2.42608775117898 \quad 3.99698330907995$

H $1.63236439050472 \quad-2.13479068755855 \quad 1.87111508708947$

$\begin{array}{llll}\text { C } & 1.39870756123156 & -3.14904627318032 & 5.11536813636145\end{array}$

H $0.08766089099827 \quad-4.71463258154299 \quad 5.83062546717061$

H $2.58425173566675 \quad-1.65428610049160 \quad 4.09710371424782$

H $1.82533130571050 \quad-2.94144125039191 \quad 6.09800610903099$

C $\quad-0.30732166916774 \quad-4.02282311510888 \quad 1.21049806858366$ 
S 39

\begin{tabular}{cccc}
$\mathrm{H}$ & -1.35575316883866 & -4.33328912257733 & 1.40476922470809 \\
$\mathrm{Fe}$ & -0.05095030714817 & -0.99875337250469 & 0.19946572555102 \\
$\mathrm{Si}$ & -1.37926836996783 & -2.87499816465772 & -1.73663781670160 \\
$\mathrm{H}$ & -1.17858350484719 & -1.42286622360586 & -1.24795856994880 \\
$\mathrm{C}$ & -2.83164776232759 & -3.81682452014443 & -1.02143158878139 \\
$\mathrm{H}$ & -3.29130518437012 & -4.39656739954519 & -1.83647811350041 \\
$\mathrm{H}$ & -2.57034332738807 & -4.50540893933780 & -0.20994913782055 \\
$\mathrm{H}$ & -3.59162378207418 & -3.12053524592583 & -0.63819736947879 \\
$\mathrm{O}$ & -0.08669938633375 & -3.81426731636546 & -2.21933179293100 \\
$\mathrm{C}$ & 0.07063655646511 & -4.29035516412617 & -3.55883035222641 \\
\hline $\mathrm{H}$ & -0.79000268094785 & -4.89802485138232 & -3.88150535151021 \\
\hline $\mathrm{H}$ & 0.18853350734119 & -3.46234316525427 & -4.27329160462522 \\
\hline $\mathrm{H}$ & 0.97071454763889 & -4.92329087445949 & -3.57778053181967 \\
$\mathrm{O}$ & -1.92970679399580 & -2.38161889554446 & -3.26744260701753 \\
$\mathrm{H}$ & -2.88831035430551 & -1.35340548418240 & -3.43430363758236 \\
\hline $\mathrm{H}$ & -3.90890436761325 & -1.69818706630980 & -3.18593114413026 \\
\hline & -2.66082198424778 & -0.47226419979423 & -2.80441772514416 \\
\hline & -2.88126450038751 & -1.03199143358817 & -4.48693922366199
\end{tabular}

\section{TS3_R}

$\mathrm{E}=-3605.59879596137$ a.u.

$\begin{array}{lllll}\text { O } & 2.39589763407743 & 0.08489361948237 & -2.30223156837914\end{array}$

$\begin{array}{llll}\text { O } & -4.45700670339987 & -0.13257651201026 & 1.51629225816757\end{array}$

$\begin{array}{lllll}\mathrm{N} & -0.28972640103543 & 0.62134561163407 & 0.95915276251274\end{array}$

N $0.65121812586193 \quad-0.93127728273780 \quad-1.25817534706356$

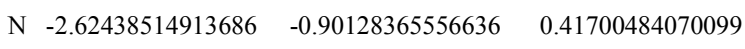

$\begin{array}{llll}\text { C } & 0.85454471314692 & 1.34713608336340 & 0.74547569502523\end{array}$

C $\quad-3.87725622431608 \quad 0.69257584769322 \quad-3.37379882197980$

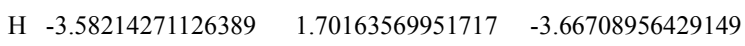

C $0.84290059441878 \quad 2.52125454394320 \quad 1.62942023441261$

$\begin{array}{llll}\text { C } & -0.39830808754896 & 2.49221434818533 & 2.31110615899337\end{array}$

C $-1.11046172147188 \quad 1.30514271869570 \quad 1.82019479690385$

C $-0.73053233746462 \quad 3.49056989837466 \quad 3.22877344488499$

H $-1.68604069095387 \quad 3.47997002592826 \quad 3.75586056530302$

$\begin{array}{llll}\text { C } & 0.19723494968144 & 4.50925459839560 & 3.46679890805510\end{array}$

H $\quad-0.03692050981423 \quad 5.29487991657599 \quad 4.18673016412273$

C $1.42837364954315 \quad 4.53747763620299 \quad 2.79103308122192$

$\begin{array}{llll}\mathrm{H} & 2.13362153567045 & 5.34447227285552 & 2.99509449522769\end{array}$

C $1.76055959184492 \quad 3.54703718790754 \quad 1.86139450122602$

H $2.71762979463036 \quad 3.57963043510700 \quad 1.33798886883830$

$\begin{array}{lllll}\text { C } & 1.77263564228395 & 1.03552621265427 & -0.23904306801777\end{array}$

$\begin{array}{llll}\text { H } & 2.63220659359984 & 1.68343811269989 & -0.39819953577973\end{array}$
C $1.56385518069316 \quad 0.02100976889096$

$\begin{array}{llll}\text { C } & 0.78925476893539 & -1.65505384665610 & -2.54610230603524\end{array}$

$\begin{array}{llll}\mathrm{H} & -0.17643121202704 & -1.62209799926076 & -3.07420765872273\end{array}$

$\begin{array}{llll}\text { C } & 1.84316853808017 & -0.80114516049461 & -3.30785710188812\end{array}$

$\begin{array}{llll}\mathrm{H} & 2.66287126334742 & -1.39609878376059 & -3.72781457372433\end{array}$

$\begin{array}{llll}\text { H } & 1.39176262460571 & -0.17893304917049 & -4.09543353790752\end{array}$

$\begin{array}{llll}\text { C } & -2.42250840837474 & 0.94049370417864 & 2.06214591297308\end{array}$

$\begin{array}{llll}\mathrm{H} & -3.02709729283256 & 1.52083887226171 & 2.75612715948922\end{array}$

$\begin{array}{llll}\text { C } & -3.10450894829171 & -0.06546786616745 & 1.32162753103113\end{array}$

$\begin{array}{llll}\text { C } & -3.76593389807800 & -1.54744026291188 & -0.25898503394112\end{array}$

H $\quad-3.59622995863976 \quad-2.63359505181265 \quad-0.30226266499626$

$\begin{array}{lllll}\text { C } & -4.94337928607013 & -1.22868159300907 & 0.69930799265548\end{array}$

$\begin{array}{lllll}\text { H } & -5.85142327136708 & -0.89202914600397 & 0.18483426894409\end{array}$

$\begin{array}{llll}\mathrm{H} & -5.17993030730114 & -2.07183658825799 & 1.36596963274021\end{array}$

$\begin{array}{llll}\text { C } & 1.21504252186452 & -3.10763448473007 & -2.42143320554647\end{array}$

$\begin{array}{llll}\text { C } & 2.14102171457671 & -3.52370490025084 & -1.45637162645774\end{array}$

$\begin{array}{llll}\text { H } & 2.48622516079914 & -2.82556527886711 & -0.69247449166037\end{array}$

$\begin{array}{llll}\text { C } & 2.60900477694129 & -4.83940840440802 & -1.45005523854937\end{array}$

$\begin{array}{lllll}\text { H } & 3.32337459042947 & -5.14949263140948 & -0.68653466925403\end{array}$

$\begin{array}{llll}\text { C } & 2.15761244230406 & -5.75436559912353 & -2.40525507637295\end{array}$

$\begin{array}{lllll}\text { H } & 2.52512806433510 & -6.78178993508026 & -2.39760975012473\end{array}$

$\begin{array}{llll}\text { C } & 1.22135497215999 & -5.35140596173794 & -3.36122550185093\end{array}$

$\begin{array}{lllll}\text { H } & 0.85072688926693 & -6.06320310696027 & -4.10083681555017\end{array}$

$\begin{array}{llll}\text { C } & 0.75275694586454 & -4.03570021737196 & -3.36576191994524\end{array}$

$\begin{array}{lllll}\mathrm{H} & 0.01797069925934 & -3.72353855058206 & -4.11255720487677\end{array}$

$\begin{array}{llll}\text { C } & -3.98353195577845 & -1.04756858720046 & -1.68098858042970\end{array}$

$\begin{array}{lllll}\text { C } & -4.61429273944867 & -1.88463762652510 & -2.61384062075547\end{array}$

$\begin{array}{llll}\mathrm{H} & -4.89529278384525 & -2.89997835130325 & -2.32247501878309\end{array}$

$\begin{array}{llll}\text { C } & -4.88090543925615 & -1.43825238578257 & -3.91013065173744\end{array}$

H $\quad-5.36934290822410 \quad-2.10389223969243 \quad-4.62390487094050$

$\begin{array}{llll}\text { C } & -4.51384285642605 & -0.14504659526332 & -4.29362335811220\end{array}$

$\begin{array}{lllll}\text { H } & -4.71795817949219 & 0.20568263263642 & -5.30652359082974\end{array}$

$\begin{array}{lllll}\text { C } & -3.61253933525594 & 0.24463821260200 & -2.07647889136615\end{array}$

$\begin{array}{lllll}\mathrm{H} & -3.10089405501717 & 0.89727332845821 & -1.36764428953661\end{array}$

$\begin{array}{lllll}\text { O } & -0.07742950053584 & -2.53019306881947 & 1.67790637450373\end{array}$

$\begin{array}{lllll}\text { C } & 0.62116555593391 & -2.62793546577479 & 2.90657934483461\end{array}$

$\begin{array}{llll}\mathrm{H} & 0.26337418045107 & -3.54885432595072 & 3.40648626646368\end{array}$

Fe $-0.60760688430370 \quad-1.30798611605707 \quad 0.34023778666407$

Si $-1.22219914204348 \quad-4.54298752819608 \quad 0.77740352439856$

H $-1.17128290952715 \quad-3.43627292853310 \quad-0.26382511184179$

$\begin{array}{llll}\text { C } & -2.48079365337574 & -4.28808335377249 & 2.14935037469270\end{array}$

$\begin{array}{llll}\mathrm{H} & -2.59662533261239 & -5.23364235709670 & 2.70113131877979\end{array}$ 


\begin{tabular}{llll} 
H & -2.20390886706882 & -3.49490497706258 & 2.85219454798970 \\
H & -3.46725522793818 & -4.04420715718643 & 1.72372447722970 \\
O & 0.19319388792772 & -5.21329820448704 & 1.31343988383973 \\
C & 0.56497623607125 & -6.57966198067585 & 1.14677525700051 \\
H & -0.06069096008402 & -7.24528225114829 & 1.76524656131239 \\
H & 0.48054979042446 & -6.90085518802578 & 0.09845626508123 \\
H & 1.61105534826161 & -6.67674882805628 & 1.47175661169989 \\
O & -1.85082018896680 & -5.80063192112227 & -0.16640856936848 \\
C & -2.85394259242377 & -5.61053350267130 & -1.14113502578591 \\
H & -3.85658134352763 & -5.49498199611598 & -0.68579776898542 \\
H & -2.65455585550784 & -4.72244889214465 & -1.77186262993486 \\
H & -2.88339480165308 & -6.49354251721785 & -1.79819561613566 \\
C & 2.12538371130442 & -2.80347129408682 & 2.71448522264461 \\
C & 2.78180169135487 & -3.91612988013742 & 3.25632504974284 \\
C & 2.89090349059112 & -1.84868787836213 & 2.02934672627485 \\
C & 4.16485757627169 & -4.07467851927380 & 3.12356175345496 \\
C & 4.27205716111732 & -2.00333272399479 & 1.88867448039135 \\
C & 4.91597657082632 & -3.11791942717168 & 2.43657951234676 \\
H & 2.19553799431097 & -4.67249129121628 & 3.78349619068570 \\
H & 2.40298997361064 & -0.97218078373101 & 1.59784836827674 \\
H & 4.65558920955615 & -4.95004676223220 & 3.55402706162154 \\
H & 4.85013517832335 & -1.24818229209835 & 1.35198523749077 \\
H & 5.99547271274620 & -3.23795016072063 & 2.32887389059219 \\
С & 0.31683822659031 & -1.44194656033908 & 3.83286434575559 \\
H & 0.79069905608313 & -1.58910334708959 & 4.81496432862824 \\
H & 0.69719777530451 & -0.502556629483410 & 3.40820768303163 \\
\hline
\end{tabular}

\section{Int3_S}

$\mathrm{E}=-3605.60356540613$ a.u.

$\begin{array}{lrrr}\text { O } & 2.79059436959520 & -0.29707443790315 & -2.03799432256812 \\ \mathrm{O} & -4.39226255618931 & -0.13427259290687 & 1.23544322831351 \\ \mathrm{~N} & -0.15994751352457 & 0.40528259930311 & 0.94551881956321 \\ \mathrm{~N} & 0.88077443923801 & -1.20491062050450 & -1.19793848271329 \\ \mathrm{~N} & -2.52877286967697 & -0.98682171160581 & 0.25811144324979 \\ \mathrm{C} & 1.05776591218313 & 1.03547695237895 & 0.85639822447754 \\ \mathrm{C} & -3.60287017623865 & 0.42373247894662 & -3.66188325427918 \\ \mathrm{H} & -3.30136794292265 & 1.42025011010919 & -3.98966446642074 \\ \mathrm{C} & 1.05106646756310 & 2.21090551255510 & 1.73979627254617 \\ \mathrm{C} & -0.25273300773298 & 2.29003105530396 & 2.28326274030788 \\ \mathrm{C} & -1.00365940386417 & 1.15720490147109 & 1.72330093050213 \\ \mathrm{C} & -0.60006812979392 & 3.32316917408152 & 3.15592718230778\end{array}$

H $\quad-1.60484230355076 \quad 3.39737270083468 \quad 3.57529070481241$

$\begin{array}{llll}\text { C } & 0.37716240241485 & 4.26615441779201 & 3.48964542395248\end{array}$

$\begin{array}{llll}\text { H } & 0.13163023732302 & 5.07736274426690 & 4.17670244464952\end{array}$

C $1.67206260073058 \quad 4.18583252301454 \quad 2.95187435357603$

H $\quad 2.41467733390563 \quad 4.93592714285370 \quad 3.22793126625831$

$\begin{array}{llll}\text { C } & 2.01995048670541 & 3.16032713023730 & 2.06659879210799\end{array}$

H $3.02673810658283 \quad 3.10762664664782 \quad 1.64912405522666$

C $2.04474483889365 \quad 0.65169362361005 \quad-0.02371302628986$

H $2.97846934862444 \quad 1.20823300444353 \quad-0.06493698846686$

$\begin{array}{llll}\text { C } & 1.85775648296046 & -0.33082349032040 & -1.04061266088271\end{array}$

C $1.01314148624511 \quad-1.76827759811464 \quad-2.57315751992539$

H $\quad 0.28620140063473 \quad-1.23855151395234 \quad-3.21412530711890$

C $2.45493533170503 \quad-1.36689790050184 \quad-2.95646265911955$

H $3.16507873532947 \quad-2.19356785861997 \quad-2.80057422545029$

H $\quad 2.55137077667188 \quad-0.98425759606743 \quad-3.97951462679201$

$\begin{array}{llll}\text { C } & -2.35366837632077 & 0.89041740365797 & 1.85917490564896\end{array}$

$\begin{array}{llll}\text { H } & -2.97030654018435 & 1.51949803874186 & 2.49763613449608\end{array}$

$\begin{array}{llll}\text { C } & -3.03213402881416 & -0.10714341617633 & 1.10693106908774\end{array}$

$\begin{array}{llll}\text { C } & -3.65043460296097 & -1.65209360088956 & -0.43481921883940\end{array}$

H $\quad-3.49705820067533 \quad-2.74102168216054 \quad-0.40550537217941$

$\begin{array}{llll}\text { C } & -4.86667358802000 & -1.25556407208175 & 0.44301519178068\end{array}$

H $\quad-5.73519971814572 \quad-0.91832067015997 \quad-0.13528972573574$

H $\quad-5.16452371542561 \quad-2.05825126388940 \quad 1.13405177666417$

$\begin{array}{llll}\text { C } & 0.77945831428680 & -3.25343252361161 & -2.73153744032607\end{array}$

C $1.44317110015851 \quad-4.18585277572470 \quad-1.92099801755790$

H $2.06233417368615 \quad-3.83756554575047 \quad-1.09217793029207$

C $1.29839159067715 \quad-5.55346220161380 \quad-2.15286263271179$

H $1.80735563924113 \quad-6.26797362490913 \quad-1.50526165957340$

$\begin{array}{llll}\text { C } & 0.49543894727799 & -6.00882475430059 & -3.20312683872505\end{array}$

H $\quad 0.38434367221774 \quad-7.07938448317714 \quad-3.38176355206521$

C $\quad-0.17566940324130 \quad-5.08851371349835 \quad-4.01039790064323$

H $\quad-0.81310260306290 \quad-5.43520918667705 \quad-4.82551072092773$

$\begin{array}{llll}\text { C } & -0.03571910782789 & -3.71832439697242 & -3.77165850321792\end{array}$

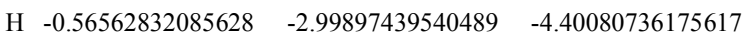

$\begin{array}{llll}\text { C } & -3.78851636958988 & -1.23016096369162 & -1.89089145795265\end{array}$

C $\quad-4.35678619209143 \quad-2.12152253090714 \quad-2.81310182824896$

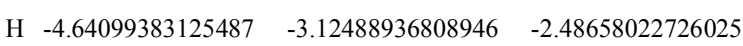

$\begin{array}{llll}\text { C } & -4.55410512467438 & -1.74402255372482 & -4.14338337388684\end{array}$

H $\quad-4.99470269480480 \quad-2.45111971351693 \quad-4.84835367542883$

$\begin{array}{llll}\text { C } & -4.17864061962719 & -0.46746279807470 & -4.57163096589284\end{array}$

H $\quad-4.32879439801739 \quad-0.17088150240145 \quad-5.61095129365893$

C $\quad-3.40823718372839 \quad 0.04513378373722 \quad-2.33066275179331$ 
S 41

\begin{tabular}{|c|c|c|c|c|c|c|c|}
\hline $\mathrm{H}$ & -2.94494476241998 & 0.74018502104225 & -1.62883386660442 & $\mathrm{~N}$ & 0.62621759155746 & -1.01105927967026 & -1.32893654643460 \\
\hline $\mathrm{O}$ & -0.01825750299633 & -2.73112653565738 & 1.77921382036434 & $\mathrm{~N}$ & -2.68928984320300 & -0.86279911641538 & 0.40546300977535 \\
\hline $\mathrm{C}$ & 0.67654173513432 & -2.57534368989144 & 3.04255665532815 & $\mathrm{C}$ & 0.86917726666758 & 1.25926050123024 & 0.66946847639415 \\
\hline 1 & 0.72778354041420 & -3.59167565771617 & 3.46904304471309 & $\mathrm{C}$ & -4.15468937123954 & 0.72388321214281 & -3.31727073154949 \\
\hline Fe & -0.53746123206107 & -1.50862068282788 & 0.28838118039273 & $\mathrm{H}$ & -3.90725178730608 & 1.74361539569214 & -3.61764804456740 \\
\hline $\mathrm{Si}$ & -0.86477472960240 & -4.34563988481322 & 1.17107739369578 & $\mathrm{C}$ & 0.91384755030298 & 2.42258297321669 & 1.57061906218181 \\
\hline $\mathrm{H}$ & -1.04348801033692 & -3.36967596092285 & -0.05830238760629 & $\mathrm{C}$ & -0.31434700332375 & 2.42820017626812 & 2.27018690017941 \\
\hline $\mathrm{C}$ & -2.26152423203235 & -4.26001308503229 & 2.45400166599052 & $\mathrm{C}$ & -1.07603001016754 & 1.27159066434660 & 1.77647181575804 \\
\hline $\mathrm{H}$ & -1.84967369172303 & -4.33257964220708 & 3.47225870870669 & $\mathrm{C}$ & -0.59984229001690 & 3.42834415318045 & 3.20119491159987 \\
\hline $\mathrm{H}$ & -2.80738450043754 & -3.30646067676046 & 2.39845632564117 & $\mathrm{H}$ & -1.54725733832043 & 3.44563739433093 & 3.74236262539725 \\
\hline $\mathrm{H}$ & -2.98074343328646 & -5.08192700624700 & 2.32663950841020 & $\mathrm{C}$ & 0.36636880127307 & 4.41238038543649 & 3.43416712817977 \\
\hline $\mathrm{O}$ & 0.52436978241851 & -5.15080649078839 & 1.69254552396451 & $\mathrm{H}$ & 0.17023405337139 & 5.19826289675649 & 4.16511169493438 \\
\hline $\mathrm{C}$ & 0.67748384980528 & -6.56523716122776 & 1.73307425527508 & $\mathrm{C}$ & 1.58682496303883 & 4.40534289244890 & 2.74026040867448 \\
\hline $\mathrm{H}$ & -0.04421192754570 & -7.03353455336033 & 2.42415375627764 & $\mathrm{H}$ & 2.32240851125900 & 5.18569901689622 & 2.94055359700540 \\
\hline $\mathrm{H}$ & 0.53727269186731 & -7.01898957870020 & 0.74100358944646 & $\mathrm{C}$ & 1.87025116586290 & 3.41269755623541 & 1.79616318826604 \\
\hline $\mathrm{H}$ & 1.69503949155296 & -6.77966542388556 & 2.09567098553610 & $\mathrm{H}$ & 2.81925071865843 & 3.41768168679045 & 1.25734648520057 \\
\hline $\mathrm{O}$ & -1.40429766553138 & -5.65937889770085 & 0.18032552632626 & $\mathrm{C}$ & 1.76682005136707 & 0.94851528959503 & -0.32910733719453 \\
\hline $\mathrm{C}$ & -2.50895191417096 & -5.53545908715727 & -0.67544816453798 & $\mathrm{H}$ & 2.63319548611750 & 1.58661085542672 & -0.49068060930506 \\
\hline $\mathrm{H}$ & -3.46432430537215 & -5.42150309525707 & -0.12070431963717 & $\mathrm{C}$ & 1.53358704529655 & -0.05792153044070 & -1.31593075127108 \\
\hline H & -2.40710907204469 & -4.66917538823848 & -1.36176016636958 & $\mathrm{C}$ & 0.77125598867560 & -1.76465936421196 & -2.60001895644643 \\
\hline $\mathrm{H}$ & -2.59113891719551 & -6.44301613766151 & -1.29529358734671 & $\mathrm{H}$ & -0.20507423321216 & -1.79217847155551 & -3.10708057329857 \\
\hline $\mathrm{C}$ & 2.11670820901287 & -2.12896555318125 & 2.80991114585966 & $\mathrm{C}$ & 1.76959853623520 & -0.88551325531124 & -3.40302415963016 \\
\hline $\mathrm{C}$ & 2.92825577095441 & -2.88952236122942 & 1.94991153233592 & $\mathrm{H}$ & 2.58796076944113 & -1.45679622679101 & -3.85620452704657 \\
\hline $\mathrm{C}$ & 2.68487995016991 & -1.02071493023673 & 3.45221772421387 & $\mathrm{H}$ & 1.27211843388594 & -0.27025713493845 & -4.16810302132512 \\
\hline $\mathrm{C}$ & 4.26250579958947 & -2.54546127089146 & 1.73387483465493 & $\mathrm{C}$ & -2.39377181183987 & 0.96035848149651 & 2.04767026171689 \\
\hline $\mathrm{C}$ & 4.02466302202792 & -0.67526756371642 & 3.23915595640479 & $\mathrm{H}$ & -2.95698277607012 & 1.55735435526272 & 2.76189524553548 \\
\hline $\mathrm{C}$ & 4.81818224090089 & -1.43404866682783 & 2.37841030621934 & $\mathrm{C}$ & -3.12548871722660 & -0.02734796278012 & 1.32952627277625 \\
\hline $\mathrm{H}$ & 2.48902189318168 & -3.76530976208339 & 1.46831767146953 & $\mathrm{C}$ & -3.86178676483682 & -1.51402746352703 & -0.21337722067018 \\
\hline $\mathrm{H}$ & 2.08486826398899 & -0.41305552021722 & 4.13025923780926 & $\mathrm{H}$ & -3.69115838216660 & -2.59944853338379 & -0.25598133509696 \\
\hline $\mathrm{H}$ & 4.87850233542522 & -3.15307718134085 & 1.06752862243707 & $\mathrm{C}$ & -4.99305152579511 & -1.18551526158707 & 0.79522707874254 \\
\hline $\mathrm{H}$ & 4.44606332882780 & 0.19132322115761 & 3.75233148967275 & $\mathrm{H}$ & -5.92305377696378 & -0.84918233349338 & 0.32186625766843 \\
\hline $\mathrm{H}$ & 5.86392122688877 & -1.16776554211974 & 2.21417692827146 & $\mathrm{H}$ & -5.19861042723030 & -2.02223901368631 & 1.47941696033778 \\
\hline $\mathrm{C}$ & -0.12778013469571 & -1.68925457683653 & 3.98924149251011 & $\mathrm{C}$ & 1.26837206538929 & -3.19122033479437 & -2.43983689509601 \\
\hline $\mathrm{H}$ & 0.32802087556511 & -1.67469747134644 & 4.99034444501622 & $\mathrm{C}$ & 2.19586510137803 & -3.54557479259491 & -1.45237989446664 \\
\hline $\mathrm{H}$ & -0.19593230730334 & -0.65622355961192 & 3.62202373266490 & $\mathrm{H}$ & 2.49682636817895 & -2.81788317485049 & -0.69742092784325 \\
\hline \multirow[t]{2}{*}{ I } & -1.14645572400457 & -2.08454619059380 & 4.08719828066518 & $\mathrm{C}$ & 2.72207929154970 & -4.83876773194460 & -1.41024069064709 \\
\hline & & & & $\mathrm{H}$ & 3.43148626438846 & -5.10299869300936 & -0.62530511964549 \\
\hline \multicolumn{4}{|c|}{ Int3_R } & $\mathrm{C}$ & 2.33085834427427 & -5.79052003708906 & -2.35506405343287 \\
\hline \multicolumn{4}{|c|}{$\mathrm{E}=-3605.60302891949$ a.u. } & $\mathrm{H}$ & 2.74158715729841 & -6.80072338527643 & -2.31815346648427 \\
\hline $\mathrm{O}$ & 2.33908472150156 & 0.01309399659485 & -2.41401147634882 & $\mathrm{C}$ & 1.39617010005175 & -5.44842987190059 & -3.33594749935061 \\
\hline $\mathrm{O}$ & -4.46619308590124 & -0.08281287175516 & 1.58261066488772 & $\mathrm{H}$ & 1.07087282724267 & -6.19079365693958 & -4.06671394312936 \\
\hline $\mathrm{v}$ & -0.29582047836988 & 0.56410173423977 & 0.89331753912294 & $\mathrm{C}$ & 0.86747430727984 & -4.15700932448634 & -3.37445234609725 \\
\hline
\end{tabular}




\begin{tabular}{|c|c|c|c|c|c|c|c|}
\hline & 0.12950834471397 & -3.89515145692890 & -4.13717326975337 & $\mathrm{H}$ & 0.56152439722248 & -1.63751687274594 & 4.74726384397907 \\
\hline $\mathrm{C}$ & -4.14104685144060 & -1.02160060652013 & -1.62642341608803 & $\mathrm{H}$ & 0.46888705438381 & -0.61250748767513 & 3.29828800532661 \\
\hline & -4.76859326119239 & -1.88117641682115 & -2.54050627831617 & $\mathrm{H}$ & -0.96801760126636 & -1.52381261961009 & 3.83249144345506 \\
\hline & -4.99851698255835 & -2.90722785776242 & -2.24297300644628 & & & & \\
\hline & -5.09308926361638 & -1.44318841223692 & -3.82656426793938 & TS 4 & & & \\
\hline & -5.57733458082789 & -2.12637869645385 & -4.52645615302567 & $\mathrm{E}=$ & -3605.59878173843 a. & & \\
\hline & -4.78806558487258 & -0.13655219646140 & -4.21827138367644 & $\mathrm{O}$ & 2.60487869523989 & 0.26408793873113 & -1.55189454364421 \\
\hline & -5.03724701131234 & 0.20728016225012 & -5.22343628308809 & $\mathrm{O}$ & -4.92404676101904 & -0.41134901548580 & 0.78553319713402 \\
\hline & -3.83204923038744 & 0.28449273192045 & -2.03044477226075 & $\mathrm{~N}$ & -0.73932924537708 & 0.45617957761723 & 1.11391418321915 \\
\hline & -3.32330333748505 & 0.95640456672316 & -1.33753917470520 & $\mathrm{~N}$ & 0.67403110390136 & -0.81145524708236 & -1.03098807759932 \\
\hline & -0.10246409543395 & -2.72003149886044 & 1.60131594105453 & $\mathrm{~N}$ & -2.89840864629527 & -0.95108819228041 & -0.08593636337733 \\
\hline & 0.55493656513454 & -2.75459110546550 & 2.89081652053301 & $\mathrm{C}$ & 0.38385871131412 & 1.24075064464847 & 1.18764909102005 \\
\hline & 0.21637472434831 & -3.67925263381829 & 3.38485354182968 & $\mathrm{C}$ & -3.53123483181554 & 0.51951765757845 & -4.08341282346010 \\
\hline & -0.69113763058463 & -1.36366095270960 & 0.24892911491685 & $\mathrm{H}$ & -3.29416112012722 & 1.55391257905733 & -4.33865683140045 \\
\hline & -0.96786819251866 & -4.24403251960692 & 0.84874051790197 & $\mathrm{C}$ & 0.15558870978519 & 2.30145617728092 & 2.17950464027215 \\
\hline & -1.16461317350783 & -3.13564626235926 & -0.28134888131163 & $\mathrm{C}$ & -1.17739664361014 & 2.15133970158327 & 2.62715271801960 \\
\hline & -2.41707025254424 & -4.31120831254080 & 2.07053004792964 & $\mathrm{C}$ & -1.73924831288348 & 1.01755792935809 & 1.87242184854042 \\
\hline & -2.52184311191376 & -5.34310648574520 & 2.44008184762180 & $\mathrm{C}$ & -1.71708068198912 & 3.01935713662326 & 3.57454486779634 \\
\hline & -2.31191227198002 & -3.64235075756794 & 2.93511528988345 & $\mathrm{H}$ & -2.74662162322973 & 2.91236111896144 & 3.92040397932313 \\
\hline & -3.36650893442836 & -4.06421801642637 & 1.56916782322639 & $\mathrm{C}$ & -0.90011263972394 & 4.03539828359985 & 4.08541053879778 \\
\hline & 0.35116613654747 & -5.14354251516498 & 1.37869583939230 & $\mathrm{H}$ & -1.29537913181827 & 4.71916740725942 & 4.83784168133897 \\
\hline & 0.51152186180407 & -6.54404919564065 & 1.19979706718903 & $\mathrm{C}$ & 0.42172379652479 & 4.18639920471546 & 3.64070197609795 \\
\hline & -0.32253639623545 & -7.11534929723112 & 1.64309343566443 & $\mathrm{H}$ & 1.03764171865608 & 4.98701068215752 & 4.05315697844385 \\
\hline & 0.57747081822188 & -6.81200747565812 & 0.13508262134272 & $\mathrm{C}$ & 0.96123882860404 & 3.32664202877537 & 2.67700803300453 \\
\hline & 1.44520780950467 & -6.83813437484728 & 1.70512227605297 & $\mathrm{H}$ & 1.98866530625120 & 3.45801230785517 & 2.33372456966480 \\
\hline & -1.47398788836480 & -5.41979736186392 & -0.31591586405685 & $\mathrm{C}$ & 1.48605715537814 & 1.06773597150831 & 0.36918431649143 \\
\hline & -2.47396033707049 & -5.18073965866287 & -1.26809028803577 & $\mathrm{H}$ & 2.33607252581627 & 1.74094060712957 & 0.46217877996121 \\
\hline & -3.49331997724264 & -5.23785180365862 & -0.83209047216390 & $\mathrm{C}$ & 1.53979130470896 & 0.13605899044255 & -0.70330262066507 \\
\hline & -2.36561721241441 & -4.18370154763905 & -1.74518030079385 & $\mathrm{C}$ & 1.06852684318148 & -1.33794104006758 & -2.36340533650107 \\
\hline & -2.40983082175967 & -5.94165119887655 & -2.06355040509079 & $\mathrm{H}$ & 0.44735387429204 & -0.82375023068314 & -3.11895306487229 \\
\hline & 2.06761978524796 & -2.84585764930313 & 2.76166790376622 & $\mathrm{C}$ & 2.53188540116994 & -0.86093167751633 & -2.46744570220823 \\
\hline & 2.75813534406536 & -3.91205702661890 & 3.35178872922728 & $\mathrm{H}$ & 3.23527934217560 & -1.63487598094188 & -2.12260474085316 \\
\hline & 2.80807150704214 & -1.85688023956187 & 2.09819947210578 & $\mathrm{H}$ & 2.81628627860456 & -0.50856097518587 & -3.46569435659784 \\
\hline & 4.15222944522411 & -3.99023393486042 & 3.28860600539486 & $\mathrm{C}$ & -3.06219556090109 & 0.63595294511388 & 1.80000195130379 \\
\hline & 4.20080242470198 & -1.93429775412803 & 2.02393851561033 & $\mathrm{H}$ & -3.80076506032684 & 1.12277806006902 & 2.43335219851228 \\
\hline & 4.87931960578043 & -3.00107959838197 & 2.62182585343453 & $\mathrm{C}$ & -3.56792717601094 & -0.26966999344993 & 0.81695048552095 \\
\hline & 2.19107193990630 & -4.69693974801774 & 3.85673251626436 & $\mathrm{C}$ & -3.86104230898182 & -1.66011497872921 & -0.94872078044013 \\
\hline 17 & 2.29424221987173 & -1.01520744800062 & 1.62939446848892 & $\mathrm{H}$ & -3.63256498698465 & -2.73709532974366 & -0.91412920080594 \\
\hline 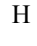 & 4.67090354909755 & -4.83005812480162 & 3.75508195224034 & $\mathrm{C}$ & -5.22837670268464 & -1.37499897389998 & -0.25950800271505 \\
\hline 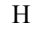 & 4.75969289935832 & -1.15573319125819 & 1.50094342237449 & $\mathrm{H}$ & -5.96597959040624 & -0.92134694417059 & -0.93363953971143 \\
\hline & 5.96772604682363 & -3.06051194309499 & 2.56705885272764 & $\mathrm{H}$ & -5.66064052064846 & -2.26519710919971 & 0.21859207283151 \\
\hline & 0.12660503160430 & -1.55777326846126 & 3.74029778986133 & $\mathrm{C}$ & 0.91478656907336 & -2.82865988498900 & -2.56388156210683 \\
\hline
\end{tabular}




$\begin{array}{lllllllll}\text { C } & 1.38419229473651 & -3.74552646270857 & -1.61172209333284 & \text { C } & 4.48431258795572 & -4.67536293550144 & 1.01030728841114 \\ \text { H } & 1.79487434927752 & -3.39042184919772 & -0.66451757197393 & \text { C } & 4.91291587095459 & -2.32814830834542 & 0.63756848146966 \\ \text { C } & 1.31047314305497 & -5.11728281552005 & -1.86064917535882 & \text { C } & 5.26432493106313 & -3.66540989070403 & 0.43719622403483 \\ \text { H } & 1.66374354800262 & -5.81738910303233 & -1.10325748153769 & \text { H } & 2.75038236778689 & -5.13075573637312 & 2.22293065859714 \\ \text { C } & 0.77372671109531 & -5.58907009056248 & -3.06228911483696 & \text { H } & 3.53229979341530 & -0.95301525444789 & 1.55670735003049 \\ \text { H } & 0.72007929218389 & -6.66209613044821 & -3.25407073776358 & \text { H } & 4.75496806409886 & -5.72309429507632 & 0.86586592310011 \\ \text { C } & 0.29554046561563 & -4.68232437636121 & -4.01169226593333 & \text { H } & 5.51987858096335 & -1.53195568636678 & 0.20197514319799 \\ \text { H } & -0.13430965205327 & -5.04231309593711 & -4.94808307818388 & \text { H } & 6.14420410194912 & -3.91984129050785 & -0.15635988848477 \\ \text { C } & 0.36280699551381 & -3.30966815900260 & -3.75970421288075 & \text { C } & 1.75983675666387 & -1.35637973677020 & 3.51678090355716 \\ \text { H } & -0.01850359831656 & -2.60084613805407 & -4.49871641062975 & \text { H } & 2.64196510537144 & -1.258066860668074 & 4.16698300703574 \\ \text { C } & -3.80951239153265 & -1.21063734072842 & -2.40001165307663 & \text { H } & 1.76056966859571 & -0.52424327407222 & 2.79969855556092 \\ \text { C } & -4.11697391053347 & -2.12323110040759 & -3.41895892766599 & \text { H } & 0.86052093264294 & -1.26557900857668 & 4.14090982419878\end{array}$

H $\quad-4.33192390841048 \quad-3.16365045474567 \quad-3.16264956373989$

$\begin{array}{llll}\text { C } & -4.14234957626079 & -1.71913646788691 & -4.75611823922900\end{array}$

H $\quad-4.38311825108047 \quad-2.44240271898937 \quad-5.53712405046179$

C $\quad-3.85088412707473 \quad-0.39425057654371 \quad-5.09200383681728$

H $\quad-3.86675292804608 \quad-0.07657360479443 \quad-6.13580015766423$

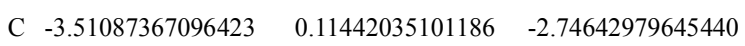

H $\quad-3.24664632059041 \quad 0.82716751034010 \quad-1.96362905793050$

$\begin{array}{llll}\text { O } & 0.57886737019213 & -2.80401310160822 & 1.96431379668287\end{array}$

$\begin{array}{llll}\text { C } & 1.75991792874649 & -2.70956632613042 & 2.81695855642118\end{array}$

H $1.68074505030326-3.50091783005317 \quad 3.57527663615482$

Fe $-0.84899557702617 \quad-1.34263518479921 \quad 0.18791897096665$

Si $-0.67672694708923 \quad-3.94274891004057 \quad 2.13380871797847$

H $\quad-1.28298576899979 \quad-2.90454284258887 \quad 0.67667178265243$

$\begin{array}{llll}\text { C } & -2.26225632529683 & -3.34655193844559 & 2.97996552067429\end{array}$

H $\quad-2.40708868324899 \quad-3.82498663270623 \quad 3.96080683131418$

$\begin{array}{llll}\mathrm{H} & -2.22363040173237 & -2.25852903818332 & 3.13550462704380\end{array}$

$\begin{array}{llll}\mathrm{H} & -3.14777230492548 & -3.55364553247673 & 2.36149644151163\end{array}$

$\begin{array}{llll}\text { O } & 0.07958119302837 & -4.87165034843072 & 3.34498181103362\end{array}$

$\begin{array}{llll}\text { C } & -0.47769100330834 & -6.07057365852565 & 3.84775086022338\end{array}$

H $\quad-1.47451141076073 \quad-5.90832383323136 \quad 4.30160238429671$

H $\quad-0.58374685227178 \quad-6.83224141254694 \quad 3.05675778964525$

H $\quad 0.18834741895100 \quad-6.46374488807284 \quad 4.63208433253225$

$\begin{array}{llll}\text { O } & -0.88184169797842 & -5.20879397864665 & 1.04588940444750\end{array}$

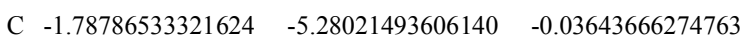

H $\quad-2.82920607902663 \quad-5.09074542881294 \quad 0.28433572286061$

H $\quad-1.53357182893548 \quad-4.56169014231598 \quad-0.83362584544882$

H $\quad-1.73848158276094 \quad-6.29809687705899 \quad-0.45239610823618$

C $2.99789428381392-3.00514491097036 \quad 1.98286092917690$

$\begin{array}{llll}\text { C } & 3.36416671306074 & -4.34550246143369 & 1.77543473323537\end{array}$

$\begin{array}{llll}\text { C } & 3.78910799255999 & -2.00258726250925 & 1.40481516200727\end{array}$

\section{TS4_R}

$\mathrm{E}=-3605.59339593519$ a.u.

O 2.09605941052953

$\begin{array}{llll}\text { O } & -4.56836588144656 & -0.30243406767145 & 1.59161935818461\end{array}$

$\begin{array}{lllll}\text { N } & -0.40963348094636 & 0.43503557183175 & 0.91425136097777\end{array}$

N $0.60408040977686 \quad-1.12221967853244 \quad-1.28763302673203$

$\begin{array}{llll}\mathrm{N} & -2.78517356673711 & -0.84215663382770 & 0.28097820486112\end{array}$

C $0.73404456223113 \quad 1.17012200437456 \quad 0.69232204356409$

$\begin{array}{llll}\text { C } & -4.29227816647198 & 1.12461109600326 & -3.22547238081537\end{array}$

H $\quad-4.06542182380252 \quad 2.17678639845190 \quad-3.40664203146564$

C $0.77438430553141 \quad 2.28437070836236 \quad 1.63998721568627$

C $-0.42753820894880 \quad 2.21595613620438 \quad 2.39007970191705$

$\begin{array}{llll}\text { C } & -1.17890359905168 & 1.08212148218311 & 1.86009566397123\end{array}$

C $-0.70711722421002 \quad 3.17251180760193 \quad 3.37156702870008$

H $-1.63498130423592 \quad 3.13939788801453 \quad 3.94541341531488$

C $0.23869054213965 \quad 4.17035683769771 \quad 3.61684526561050$

$\begin{array}{llll}\mathrm{H} & 0.04808793179530 & 4.91569620348627 & 4.39064924514775\end{array}$

C $1.43748924634216 \quad 4.22920689777833 \quad 2.88212118419182$

H $2.15715105225371 \quad 5.02174342622233 \quad 3.09249680593401$

$\begin{array}{lllll}\text { C } & 1.71303183116014 & 3.29075736749007 & 1.88480678493045\end{array}$

H $2.64073011392014 \quad 3.34986646840499 \quad 1.31267523925427$

C $1.62372511850375 \quad 0.92334321896034 \quad-0.34258924751355$

H $2.43106657394908 \quad 1.62805682695469 \quad-0.53069687124953$

C $1.40790562746704 \quad-0.06898788572211 \quad-1.32921383729015$

C $0.70363770626301 \quad-1.82577423149665 \quad-2.58761959439404$

H $\quad-0.30743534764539 \quad-1.94776414731976 \quad-3.00270758931350$

C $1.51950434742402 \quad-0.82730957915669-3.45392197955328$

H $2.33748871674018 \quad-1.29820454678834 \quad-4.01200724292958$

H $0.88244674262536 \quad-0.24852560928611 \quad-4.14048078844229$ 
S 44

\begin{tabular}{|c|c|c|c|c|c|c|c|}
\hline$c$ & -2.50344015607050 & 0.75488612215767 & 2.12363610861498 & $\mathrm{H}$ & -0.23868712414171 & -6.91317585556390 & 3.97228909515857 \\
\hline $\mathrm{H}$ & -3.04769506696601 & 1.27903409557155 & 2.90647487858013 & $\mathrm{O}$ & -0.38688530999373 & -5.28871314113743 & 0.29454069383113 \\
\hline $\mathrm{C}$ & -3.23339825913305 & -0.14630237794445 & 1.32229455797281 & $\mathrm{C}$ & -0.97133117387380 & -5.55476895268303 & -0.97472447615753 \\
\hline $\mathrm{C}$ & -3.94250303219991 & -1.44872537016538 & -0.40115318923481 & $\mathrm{H}$ & -1.82049843841837 & -6.25249828811658 & -0.87477485294662 \\
\hline $\mathrm{H}$ & -3.74111372927833 & -2.51504700456577 & -0.57129730044150 & $\mathrm{H}$ & -1.31795279284207 & -4.62421451564775 & -1.45331721431089 \\
\hline $\mathrm{C}$ & -5.08267533419972 & -1.26874251127634 & 0.63865204607359 & $\mathrm{H}$ & -0.20396864903735 & -6.01760238839549 & -1.60925993857294 \\
\hline H & -6.00626831471679 & -0.86177306054306 & 0.20804324187475 & $\mathrm{C}$ & 2.23794775427382 & -2.84634776609143 & 2.52258385513076 \\
\hline H & -5.30276468662116 & -2.20072547330415 & 1.18020617974987 & $\mathrm{C}$ & 2.90772958528455 & -4.04756928388512 & 2.80026055363723 \\
\hline $\mathrm{C}$ & 1.36524123558387 & -3.19460247779846 & -2.53880538669282 & $\mathrm{C}$ & 2.94254423697169 & -1.82154726731524 & 1.87307623950335 \\
\hline C & 2.21895995834310 & -3.58344248095148 & -1.50263675691912 & $\mathrm{C}$ & 4.24780287240913 & -4.22226570904775 & 2.44509375903775 \\
\hline $\mathrm{H}$ & 2.35917284241825 & -2.92821234501979 & -0.64269704208182 & $\mathrm{C}$ & 4.28045732076280 & -1.99477797545290 & 1.51100681618166 \\
\hline $\mathrm{C}$ & 2.88108418172437 & -4.81408704486409 & -1.55563488705489 & $\mathrm{C}$ & 4.93912562567127 & -3.19427721531007 & 1.79877703016928 \\
\hline H & 3.53461476052069 & -5.10409795626181 & -0.73186130361277 & $\mathrm{H}$ & 2.36339641466260 & -4.85284839918274 & 3.29739426982698 \\
\hline $\mathrm{C}$ & 2.70316657981682 & -5.66619535604771 & -2.64745300201040 & $\mathrm{H}$ & 2.44830706567596 & -0.87696193907786 & 1.64038867293886 \\
\hline H & 3.22210138861823 & -6.62541101496333 & -2.68687033127309 & $\mathrm{H}$ & 4.75289683594261 & -5.16209576214404 & 2.67561924442728 \\
\hline $\mathrm{C}$ & 1.84718936854277 & -5.28665727052255 & -3.68712533644526 & $\mathrm{H}$ & 4.81182980513205 & -1.18702347483982 & 1.00475377004373 \\
\hline $\mathrm{H}$ & 1.69259808860430 & -5.94928389045079 & -4.54071369816956 & $\mathrm{H}$ & 5.98661158670247 & -3.32523507933588 & 1.52178159200225 \\
\hline $\mathrm{C}$ & 1.18024015354562 & -4.06220130473041 & -3.62768192430639 & $\mathrm{C}$ & 0.49428438671147 & -1.37180831438381 & 3.66064362659070 \\
\hline $\mathrm{H}$ & 0.50420569311377 & -3.77518774567421 & -4.43789266640280 & $\mathrm{H}$ & 1.09418425950384 & -1.31150145511483 & 4.58050633427105 \\
\hline $\mathrm{C}$ & -4.23976251944489 & -0.80308862352824 & -1.74707199988432 & $\mathrm{H}$ & 0.73558012142918 & -0.50168561501009 & 3.04065396538259 \\
\hline $\mathrm{C}$ & -4.85003986302765 & -1.56412363635988 & -2.75486752489063 & $\mathrm{H}$ & -0.56751051654726 & -1.31957134520567 & 3.93620927627999 \\
\hline
\end{tabular}

H $\quad-5.05848128347233 \quad-2.62254476362543 \quad-2.57785748292496$

$\begin{array}{llll}\text { C } & -5.18594597123123 & -0.98813075073428 & -3.98252691870049\end{array}$

H $\quad-5.65682688985027 \quad-1.59616597379012 \quad-4.75704544746305$

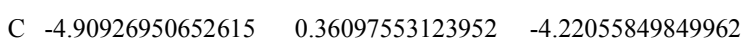

H $\quad-5.16682651236658 \quad 0.81320635689809 \quad-5.17966815188940$

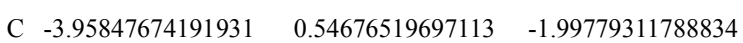

H $\quad-3.46012326718852 \quad 1.14201776357725 \quad-1.23123026042876$

$\begin{array}{llll}\text { O } & -0.13070025437762 & -2.86049273943545 & 1.80343267377025\end{array}$

C $0.79508562051953 \quad-2.68672805768836 \quad 2.96185765357038$

$\mathrm{H} \quad 0.56531409405440 \quad-3.51121310620305 \quad 3.64619379815445$

Fe $-0.83840930200999 \quad-1.42314922640398 \quad 0.14800275656817$

Si $-0.93960422570615 \quad-4.33527969061728 \quad 1.51986378889043$

H $-1.32016441017833 \quad-2.89815838758497 \quad-0.38629090764919$

$\begin{array}{llll}\text { C } & -2.80821074022759 & -4.22466973674542 & 1.63536554008016\end{array}$

$\begin{array}{llll}\mathrm{H} & -3.19301744864215 & -5.06078238724563 & 2.24050645064990\end{array}$

H $\quad-3.10834679505139 \quad-3.28965328612514 \quad 2.12744615148404$

H $\quad-3.28731332801412 \quad-4.266271167482890 .64921882288057$

O $-0.44288089240224 \quad-5.19345351322506 \quad 2.87373558271317$

$\begin{array}{lllll}\text { C } & -0.69646027612439 & -6.58387486758756 & 3.02803505865541\end{array}$

H $-1.77934699459702 \quad-6.79909473881296 \quad 3.08044829527687$

$\begin{array}{llll}\text { H } & -0.26427029729711 & -7.16474874069123 & 2.19779743880557\end{array}$ 


\section{References:}

(1) Armarego, W. L. F.; Chai, C. Purification of Laboratory Chemicals, Seventh Edition; Butterworth-Heinemann: Amsterdam : London, 2012.

(2) Bleith, T.; Wadepohl, H.; Gade, L. H. J. Am. Chem. Soc. 2015, 137, 2456-2459.

(3) Deng, Q.-H.; Wadepohl, H.; Gade, L. H. Chem. - Eur. J. 2011, 17, 1492214928.

(4) Wu, J. Y.; Stanzl, B. N.; Ritter, T. J. Am. Chem. Soc. 2010, 132, 13214-13216.

(5) Deng, Q.-H.; Bleith, T.; Wadepohl, H.; Gade, L. H. J. Am. Chem. Soc. 2013, 135, 5356-5359.

(6) Enholm, E. J.; Jia, Z. J. J. Org. Chem. 1997, 62, 9159-9164.

(7) Ranu, B. C.; Banerjee, S. Eur. J. Org. Chem. 2006, 2006, 3012-3015.

(8) Yau, H. M.; Croft, A. K.; Harper, J. B. Chem. Commun. 2012, 48, 8937-8939.

(9) McDaniel, D. H.; Brown, H. C. J. Org. Chem. 1958, 23, 420-427.

(10) Hansch, C.; Leo, A.; Taft, R. W. Chem. Rev. 1991, 91, 165-195.

(11) Evans, D. F. J. Chem. Soc. Resumed 1959, 2003-2005.

(12) Grant, D. H. J. Chem. Educ. 1995, 72, 39.

(13) Piguet, C. J. Chem. Educ. 1997, 74, 815.

(14) Neese, F. Wiley Interdiscip. Rev. Comput. Mol. Sci. 2012, 2, 73-78.

(15) Becke, A. D. Phys. Rev. A 1988, 38, 3098-3100.

(16) Perdew, J. P. Phys. Rev. B 1986, 33, 8822-8824.

(17) Weigend, F.; Ahlrichs, R. Phys. Chem. Chem. Phys. 2005, 7, 3297-3305.

(18) Weigend, F. Phys. Chem. Chem. Phys. 2006, 8, 1057-1065.

(19) Weigend, F.; Häser, M.; Patzelt, H.; Ahlrichs, R. Chem. Phys. Lett. 1998, 294, $143-152$.

(20) Watson, L.; Eisenstein, O. J. Chem. Educ. 2002, 79, 1269.

(21) Becke, A. D. J. Chem. Phys. 1993, 98, 1372-1377.

(22) Becke, A. D. J. Chem. Phys. 1993, 98, 5648-5652.

(23) Lee, C.; Yang, W.; Parr, R. G. Phys. Rev. B 1988, 37, 785-789.

(24) Ernzerhof, M.; Scuseria, G. E. J. Chem. Phys. 1999, 110, 5029-5036.

(25) Staroverov, V. N.; Scuseria, G. E.; Tao, J.; Perdew, J. P. J. Chem. Phys. 2003, 119, 12129-12137.

(26) Staroverov, V. N.; Scuseria, G. E.; Tao, J.; Perdew, J. P. J. Chem. Phys. 2004, 121, 11507-11507.

(27) A. Klamt, G. Schüürmann. J Chem Soc Perkin Trans 2 1993, 799-805.

(28) Sinnecker, S.; Rajendran, A.; Klamt, A.; Diedenhofen, M.; Neese, F. J. Phys. Chem. A 2006, 110, 2235-2245.

(29) Neese, F.; Wennmohs, F.; Hansen, A.; Becker, U. Chem. Phys. 2009, 356, 98 109.

(30) Neese, F. Coord. Chem. Rev. 2009, 253, 526-563.

(31) Weymuth, T.; Couzijn, E. P. A.; Chen, P.; Reiher, M. J. Chem. Theory Comput. 2014, 10, 3092-3103.

(32) Rohmann, K.; Hölscher, M.; Leitner, W. J. Am. Chem. Soc. 2016, 138 (1), 433443.

(33) Retrieved from http://www.chemcraftprog.com. 2015. 
Fe(boxmi)OAc-d $\mathrm{d}_{3}\left(3-\mathrm{D}_{3}\right)^{2} \mathrm{H}$ NMR (92.12 MHz, $\left.\mathrm{CDCl}_{3}, 295 \mathrm{~K}\right)$ :

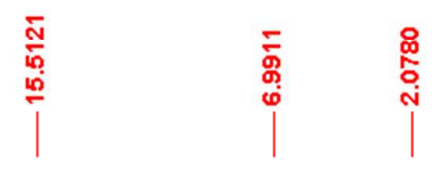

(1) 
(EtO) ${ }_{2} \mathrm{MeSiD},{ }^{1} \mathrm{H}$ NMR (600.13 $\left.\mathrm{MHz}, \mathrm{CDCl}_{3}, 295 \mathrm{~K}\right)$ :

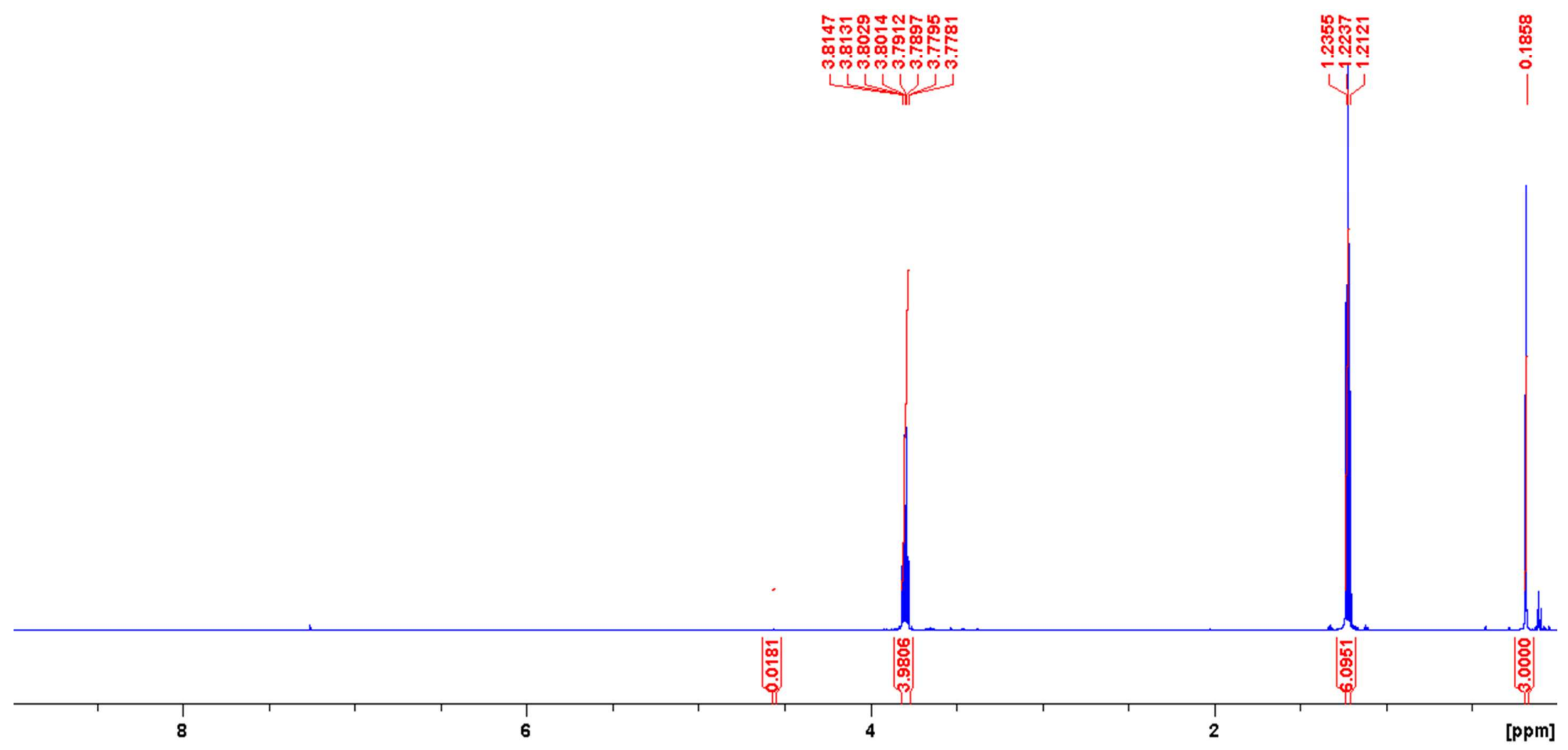


(EtO) ${ }_{2} \mathrm{MeSiD},{ }^{13} \mathrm{C}$ NMR (150.92 $\left.\mathrm{MHz}, \mathrm{CDCl}_{3}, 295 \mathrm{~K}\right)$ :
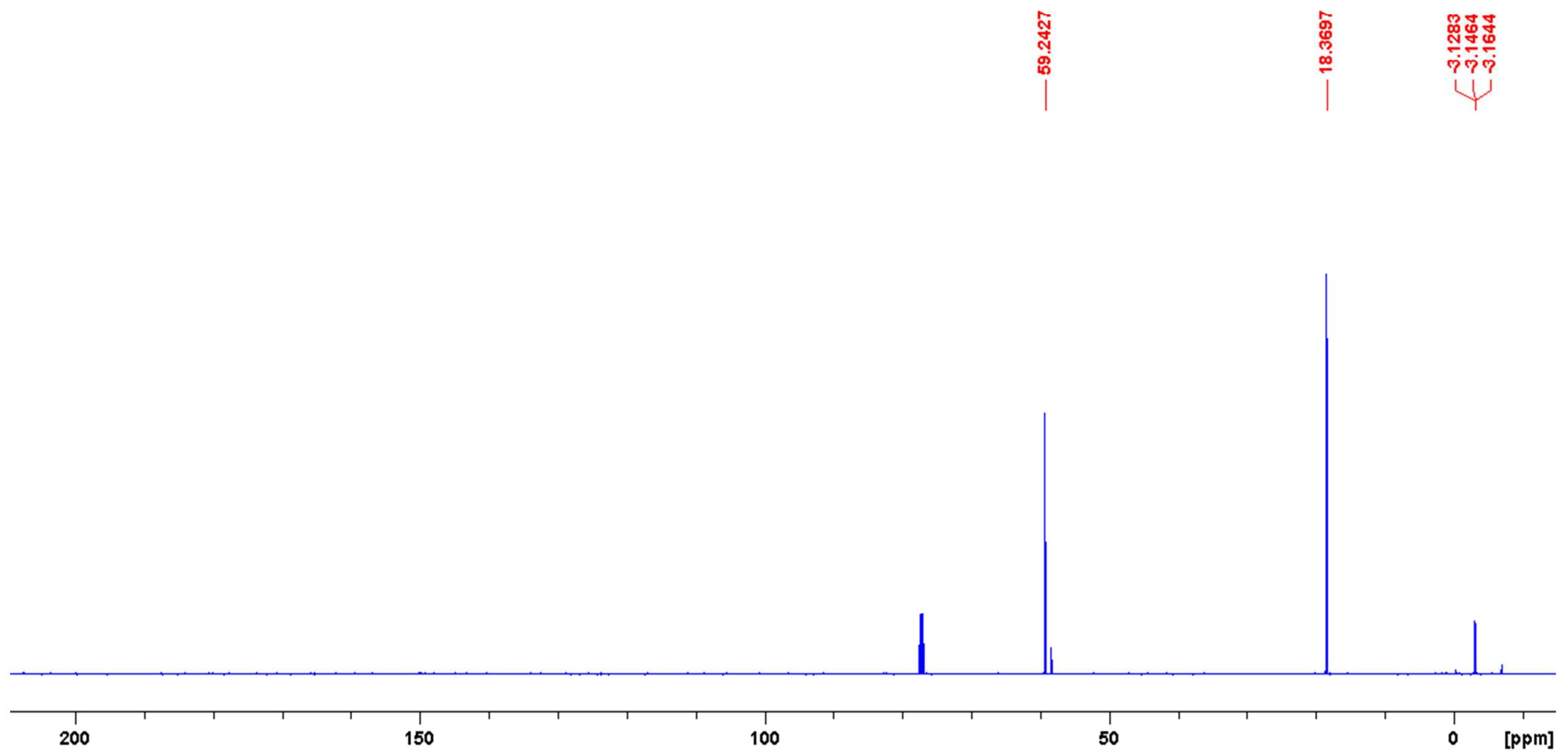